A numerical ice sheet

- ice shelf 3D

velocity field solution

T. J. Reerink et al.

\section{Derivation of a numerical solution of the 3D coupled velocity field for an ice sheet - ice shelf system, incorporating both full and approximate stress solutions}

\section{T. J. Reerink, R. S. W. van de Wal, and P.-P. Borsboom}

Institute for Marine and Atmospheric Research Utrecht, Utrecht University, The Netherlands Received: 12 December 2008 - Accepted: 13 January 2009 - Published: 6 February 2009 Correspondence to: T. J. Reerink (t.reerink@uu.nl)

Published by Copernicus Publications on behalf of the European Geosciences Union.
Title Page

Abstract Introduction

Conclusions

Tables References Figures

14 I

4

Back

Close

Full Screen / Esc

Printer-friendly Version

Interactive Discussion 


\section{Abstract}

To overcome the mechanical coupling of an ice sheet with an ice shelf, one single set of velocity equations is presented covering both the sheet and the shelf. This set is obtained by applying shared sheet-shelf approximations. The hydrostatic approximation 5 and a constant density are the only approximations that are applied to the full-Stokes momentum equations. The remaining stress terms from the momentum equations and the stress terms from the usual ice-flow law are multiplied by coefficients which can be put to zero or one, facilitating several stress approximations per domain within one model. In addition we derived a matrix format for the discretized set of the fully coupled velocity equations on a three-dimensional vertically scaled grid, in which all linear derivative terms are treated implicitly. The compact vector format of this sparse matrix equation is developed, including the boundary conditions.

\section{Introduction}

Large ice caps, like e.g. Antarctica, roughly consist of two domains: the sheet and the shelf (see Fig. 1). The sheet or the grounded area, is that part of the ice cap where the ice rests on the bedrock. Sheet parts with their bottom below sea level are called marine sheets. Shelves are the floating parts, surrounding the sheet. Shelves are fed by ice flow from the sheet, and calve at the seaward front. The grounding line, being part of the sheet, separates the sheet from the shelf or the ocean. The part of the sheet near and including the grounding line is called the transition zone. Ice stream regions are grounded areas with a relative high sliding velocity.

Accurate modelling of the progressive growth of the sheet due to grounding shelves, or the desintegration of the sheet vice versa, requires a dynamic inclusion of these shelves and of grounding line migration. Large marine sheets can disintegrate rapidly if they become unstable due to changes in the stress regime within the ice. Those changes can be caused by an increased ice temperature, by a decline of a bordering
GMDD

2, 81-158, 2009

A numerical ice sheet - ice shelf 3D velocity field solution

T. J. Reerink et al.

Title Page

Abstract

Introduction

Conclusions

Tables

References

Figures

14

4

Back

Close

\section{Full Screen / Esc}

Printer-friendly Version

Interactive Discussion 
shelf, or by an increased sea level. For example, the scenario in which the collapse of the West Antarctic ice sheet has been proposed (e.g. Mercer, 1978), yielding a sea level rise of 5 meter, has ever since then also been challenged (e.g. Vaughan, 2007).

In order to be suitable for studying long term paleo ice-sheet evolution, a model must 5 be capable of handling various different ice-sheet configurations: from an ice free situation to a situation where continents are covered by a $4 \mathrm{~km}$ thick ice cap. The complex climate system, including ice caps themselves, generates positive feedback mechanisms which can trigger strong forcing causing dramatic expansion or retreat of the ice caps. This is indicated by various deep sea $\delta^{18} \mathrm{O}$ Cenozoic records (Zachos et al., 10 2001) representing continental ice storage and changes in deepwater temperature.

To simulate these phenomena, an ice model has to follow the forcing adequately because of the hysteresis involved with grounding line migration (Schoof, 2007). If the model reacts too slowly, it will possibly stay in a wrong stage for a long time due to this hysteresis. In addition a proper grounding line migration is important on shorter time 15 scales because of its implications on the local stress regime and the sheet extent. Not including the shelves leads to artificial hysteresis: once a part of the sheet has vanished it is difficult to recover. If a shelf forms first, it can slowly grow until the floatation criterion is exceeded. Then it grounds and becomes part of the sheet. Therefore shelves should be included to describe the irreversible behaviour of the sheet-shelf system.

Compared to the bottom friction at the sheet the bottom friction at the shelf is negligible, leading to a different stress regime within the shelf. This, combined with some other shelf specific assumptions, leads to a set of velocity equations for the shelf (see e.g. Huybrechts (1991)) which differ from the usual ones for the sheet which are based on the shallow ice approximation (SIA, Hutter (1983)). In the transition zone, which 25 is often taken equal to the grounding line, usually a more complex hybrid approach is followed (Huybrechts, 1991).

Calculating the velocity field is the central problem in ice dynamics. Once the velocity field is known, the ice fluxes can be calculated directly from mass conservation. The flow parameter for ice $A_{\text {flow }}$, partly causing the non-linearity in the velocity expres-

\section{GMDD}

2, 81-158, 2009

A numerical ice sheet - ice shelf 3D velocity field solution

T. J. Reerink et al.

Title Page

Abstract

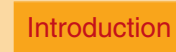

Conclusions

Tables

References

Figures

14

$\rightarrow$

4

Back

Close

Full Screen / Esc

Printer-friendly Version

Interactive Discussion 
sion, is strongly dependent on temperature (Paterson, 1994, chapter 5). By taking energy conservation into account a parallel calculation of the temperature field can be performed estimating $A_{\text {flow }}$ at each grid point.

A difficult task in ice-cap modelling is coupling the sheet and the shelf. These two 5 domains with their different set of velocity equations have to be coupled at the grounding line, which has an irregular pattern and varies in time. In addition, often a special hybrid stress treatment is used at this grounding line. Combining those difficulties at the grounding line, makes grounding line migration a delicate subject. The importance and the difficulty of modelling grounding line migration is for instance discussed by Vieli 10 and Payne (2005).

Although the SIA suffices for most parts of the sheet, in the transition zone more stresses should be included. Therefore, higher-order and full-Stokes ice-sheet models are applied to sheets, and to marine sheets in particular. The performance of a variety of such models is compared by Pattyn et al. (2008). The precise stress regime at the sheet edges possibly influences a large part of the up stream sheet. Possibly this is even more the case in the numeric situation than in reality.

For a marine sheet with a one-dimensional flow direction, an adapting grid in that direction improves the performance of modelling the grounding line migration (Schoof, 2007; van Tuyll et al., 2007). But in case of a large sheet with a grounding line varying irregularly in both horizontal directions and in time, adapting grids are hard to handle numerically.

Therefore it is necessary to develop an ice model which is robust for various ice-cap configurations, which includes shelves, and which is based on a full stress approach. Here, we derive one set of velocity equations, which is valid for the sheet, the shelf and the transition zone. Our method resembles the higher-order stress approach for the sheet of Pattyn (2003), but is extended by taking the full horizontal shear into account. Solving this single set of equations for the entire domain avoids mechanical coupling of the sheet with the shelf at an irregularly varying internal boundary. As a consequence of including most stresses in our derivation, the transition zone and the ice divide are

GMDD

2, 81-158, 2009

A numerical ice sheet - ice shelf 3D velocity field solution

T. J. Reerink et al.

Title Page

Abstract

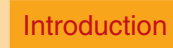

Conclusions

Tables

References

Figures

14

-1

4

Back

Close

Full Screen / Esc

Printer-friendly Version

Interactive Discussion 
described accurately, which is not the case in the SIA. Crucial in our approach is that the focus of simultaneous problems interfering at the grounding line reduces. With the proposed approach we obtain a three-dimensional (3D) description of the sheet and the shelf, with only boundary conditions at the outer domain edges, avoiding the 5 internal varying boundary conditions at the grounding line.

To obtain the general velocity equations for the sheet, the transition zone and the shelf, we applied five general assumptions to the full-Stokes momentum equations and substituted the full stress flow law of ice. We took a flow law which is based on the relation between the second invariants only. The result can be expressed in terms of 10 velocities, stresses or strain rates. Each representation has its advantages and disadvantages in applying successive approximations in an earlier or later stage. To combine the advantages of the different representations we took the velocity representation and introduced some "simplification coefficients", either one or zero, facilitating several stress approximations in a later stage. These simplification coefficients are also helpful 15 The simplification coefficients allow tests concerning the validity of the usual stress approximations, because the coefficients can be chosen separately for the sheet and the shelf. We prefer the velocity representation because the bottom boundary conditions, such as sliding, can be specified readily.

As a result we obtain a higher order stress solution for a 3D 3-component velocity field which is valid for both the sheet and the shelf. Besides the hydrostatic approximation in the vertical, all stresses are taken into account. We present a matrix format for the discretized set of fully coupled velocity equations on a 3D vertically scaled grid, in which all linear derivatives are taken implicit. The compact vector format of this sparse matrix equation is presented including the boundary conditions. The set of velocity equations can be solved with a sparse matrix solver by using this compact vector format. Section 2 gives a conceptual outline of our approach.

\section{GMDD}

2, 81-158, 2009

A numerical ice sheet - ice shelf 3D velocity field solution

T. J. Reerink et al.

Title Page

Abstract

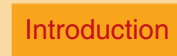

Conclusions

Tables

References

Figures

14

$\rightarrow$

4

Back

Close

Full Screen / Esc

Printer-friendly Version

Interactive Discussion 


\section{Approach}

The general approach in the work presented is characterized by the following steps:

- Derivation of the velocity equations and application of the physical approximations, see Sect. 3 and Sect. 4.

- Transformation of these velocity equations to the scaled coordinates, see Sect. 5 and Appendix B which is summarized by Table 3 .

- Decision about the implicit/explicit treatment of the equation terms, see Sect. 5. This choice strongly determines the complexity in later steps.

- Discretization of the implicit parts, see Sect. 5 and Appendix C which is summarized by Table 4 .

- Choosing a matrix format which is suited for the required "coupled dimensions". This matrix format must be capable of solving the given coupled set of equations. Its complexity depends on the relation between the discretized equations. As soon as all variables have discrete derivatives all equations become coupled, see Sect. 5 and Appendix E.

- Choosing the format to translate the sparse matrix to a three-vector format, and choosing a matching format for the right-hand side (RHS) and the solution vector, see Sect. 5 and Appendix E.

- Solving this \{three-vector matrix, RHS, solution\} -format by a sparse matrix solver. The solution contains the 3D fields of the three velocity components.

GMDD

2, 81-158, 2009

A numerical ice sheet - ice shelf 3D velocity field solution

T. J. Reerink et al.

Title Page

Abstract

Introduction

Conclusions

Tables

References

Figures

14

I

4

Back

Close

Full Screen / Esc

Printer-friendly Version

Interactive Discussion 


\section{Basic equations}

For an overview of frequently used variables and coordinates see Table 1.

In ice dynamics several different physical processes are involved. As soon as there is a gradient in the ice surface height $H_{s}$, ice starts to flow in the down slope direction 5 due to gravity. Therefore the gradient of $H_{s}$ plays an important role in the flow of ice. $H_{s}$ depends on the floatation criterion and has to be calculated differently for the sheet and the shelf with

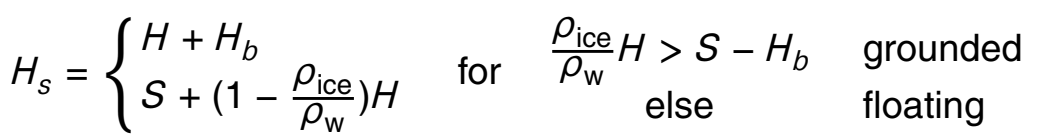

where $H$ is the ice thickness, $H_{b}$ is the bedrock level or bottom topography, $\rho_{\text {ice }}$ and $\rho_{\mathrm{w}}$ 10 are the ice and sea-water densities respectively and $S$ is the sea level. $H_{S}, H_{b}$ and $S$ are relative to present day sea level, as schematically indicated in Fig. 1 . The floating and grounded points are distinguished by the floatation criterion, which is given by the condition in Eq. (3.1).

Gravity causes deformational ice flow and, if the bottom friction is low enough, slid15 ing. The water-saturated marine sheet parts in the coastal zone experience an upward buoyancy force proportional to the ice column below sea level, like glaciers in case of a high melt water flux. This buoyancy effect might have a significant impact on the stress regime reducing the bottom friction, possibly towards the temperature dependent sliding threshold.

20 The deformation and the sliding threshold depend on the local temperature and the local pressure melting point, because higher temperatures will soften the ice and vice versa. The ice temperature $T$ depends on the surface temperature, the geothermal heat flux, the ice conductivity and the ice heat capacity, the internal deformational heating, the frictional heating at the bottom, and on heat advection.

25 The surface mass balance and the bottom shelf melt are determined by the surrounding atmosphere-ocean climate system. This surface mass balance, the bottom

GMDD

2, 81-158, 2009

A numerical ice sheet - ice shelf 3D velocity field solution

T. J. Reerink et al.

Title Page

Abstract Introduction

Conclusions

Tables

References

Figures

14

1

4

Back

Close

Full Screen / Esc

Printer-friendly Version

Interactive Discussion 
shelf melt, the geothermal heat flux and the surface ice temperature are the forcings of the ice cap. The sea level can be considered as a forcing too because of the large impact on the stress regime in the ice as soon as the floatation criterion is exceeded.

Crucial in the evolution of an ice sheet is the continental shape, the bedrock geome5 try, with its response to the ice load. The (initial) continental shape is as determing as the other forcings and in paleo studies poorly known.

\subsection{Continuity equation for ice}

The continuity equation for ice, describing mass conservation, per unit area $\Delta x \Delta y$ is

$$
\mathrm{d}_{t} H=-\nabla \cdot(\overline{\boldsymbol{v}} H)+M_{s}+M_{b}
$$

10 with $\overline{\boldsymbol{V}}$ the two-dimensional vertical averaged velocity field in $x$ and $y$ direction, $M_{s}$ the surface mass balance (in meter ice equivalent per year) being equal to the snow accumulation minus the surface melt if any, and $M_{b}$ the basal mass balance (in meter ice equivalent per year) at the shelf being equal to the refreezing ocean water minus the basal melt (relative large) and at the sheet being equal to the basal melt (relative small). The basal melt process at the shelf bottom is caused by ocean water contact, while at the sheet bottom the melt is caused by the geothermal heat flux, frictional heating and deformational heating.

This section addresses the central problem in Eq. (3.2): solving the velocity field from the Stokes momentum equations and the incompressibility condition, in combination with the ice flow. Via the flow parameter, the velocity field depends on the temperature. Further, the velocity field depends on the spatial derivatives of the surface height and indirectly on the floatation criterion. The latter is because the floating criterion determines the surface height and the bottom boundary condition, which again is temperature dependent in the grounded case due to the sliding threshold. In the next sheet-shelf assumptions to the momentum equations.

GMDD

2, 81-158, 2009

A numerical ice sheet - ice shelf 3D velocity field solution

T. J. Reerink et al.

Title Page

Abstract Introduction

Conclusions

Tables

References

Figures

14

I

4

Back

Close

\section{Full Screen / Esc}

Printer-friendly Version

Interactive Discussion 
Summation of all forces, i.e. volume forces and stresses, the second law of Newton describes conservation of momentum (see e.g. Acheson, 2003) and reads in component form (Navier-Stokes)

${ }_{5} \quad F_{\mathrm{i}}+\partial_{\mathrm{j}} \tau_{\mathrm{ij}}=\rho_{\text {ice }} \mathrm{d}_{t} v_{\mathrm{i}}$

Here, $F_{\mathrm{i}}$ is the $\mathrm{i}$-th component of the sum of the volume forces, $\rho_{\text {ice }}$ is the ice density, $\mathrm{d}_{t} v_{\mathrm{i}}$ is the time derivative of the $\mathrm{i}$-th velocity component, and $\tau_{\mathrm{ij}}$ is the Cauchy stress tensor describing the stress in the direction $i$ and acting on the plane which has $j$ as its normal vector. $\tau_{\mathrm{ij}}$ describes the normal stress components for $\mathrm{i}=\mathrm{j}$ and the shear 10 stress components for $\mathrm{i} \neq \mathrm{j}$. Because the antisymmetric part of a second order tensor only represents rotation, and because for our purpose we neglect rotation within the ice-cap system, the stress tensor is a symmetric tensor.

\subsection{The deviatoric stress equations for ice}

In this section five general approximations are made which are assumed to be valid for 15 both the sheet and the shelf (and in the transition zone). In ice dynamics the accelerations in Eq. (3.3) are neglected

$\mathrm{d}_{t} v_{\mathrm{i}}=0$

This is the first general assumption. Gravity is the permanent present volume force. Another volume force acting on water-saturated ice layers is buoyancy, which also is

caused by gravity due to the differences in the densitiy of ice and water. Both gravity and buoyancy are directed vertically, which means $F_{\mathrm{x}}=F_{\mathrm{y}}=0$. For a non-saturated ice sheet buoyancy plays no role. For an ice shelf which is in floating equilibrium the mass of the layers on top of a certain layer $z$ determines the local pressure at that $z$. Here, we do not consider the (partial) saturatation of marine ice sheets, because the ice- sheet saturation distribution would be an issue of large uncertainty. Therefore, we can

\section{GMDD}

2, 81-158, 2009

A numerical ice sheet - ice shelf 3D velocity field solution

T. J. Reerink et al.

Title Page

Abstract Introduction

Conclusions

Tables

References

Figures

14

$\rightarrow 1$

4

Back

Close

\section{Full Screen / Esc}

Printer-friendly Version

Interactive Discussion 
discount the upward buoyancy volume force $\left(F_{\mathrm{b}}=\left(\rho_{\mathrm{w}}-\rho_{\text {ice }}\right) g\right)$. Taking only gravity into account we get

$F_{\mathrm{z}}(z)=-\rho_{\text {ice }}(z) g$

GMDD

with the gravitational acceleration $g=9.81 \mathrm{~m} / \mathrm{s}^{2}$. A second general assumption 5 is made by choosing the ice density independent of $z$ at a constant value of $\rho_{\text {ice }}=910 \mathrm{~kg} / \mathrm{m}^{3}$. So we have

$F_{\mathrm{i}}=-\rho_{\mathrm{ice}} g \delta_{\mathrm{iz}}$

These two assumptions, combined with the second law of Newton (3.3) lead to the Euler equation which describes conservation of momentum for the specific case of an 10 ice sheet, an ice shelf, or a glacier:

$\partial_{\mathrm{j}} \tau_{\mathrm{ij}}=\rho_{\mathrm{ice}} g \delta_{\mathrm{iz}}$

For each component separately this becomes

$\partial_{x} \tau_{\mathrm{xx}}+\partial_{y} \tau_{\mathrm{xy}}+\partial_{z} \tau_{\mathrm{xz}}=0$

$\partial_{x} \tau_{\mathrm{yx}}+\partial_{y} \tau_{\mathrm{yy}}+\partial_{z} \tau_{\mathrm{yz}}=0$

$15 \quad \partial_{x} \tau_{\mathrm{zx}}+\partial_{y} \tau_{\mathrm{zy}}+\partial_{z} \tau_{\mathrm{zz}}=\rho_{\mathrm{ice}} g$

Where $\tau_{\mathrm{xz}}$ and $\tau_{\mathrm{yz}}$, the stresses in respectively the $\mathrm{x}$ and the $\mathrm{y}$ direction in the horizontal plane, are the horizontal shear stresses. The stresses $\tau_{\mathrm{zx}}$ and $\tau_{\mathrm{zy}}$, directed into the $\mathrm{z}$ direction and situated in the vertical planes, are called the vertical shear stresses. We will call $\tau_{\mathrm{xy}}$ and $\tau_{\mathrm{yx}}$, the stresses in respectively the $\mathrm{x}$ and the $\mathrm{y}$ direction and situated 20 in the vertical planes, the side stresses. Furthermore, $\tau_{\mathrm{xx}}$ and $\tau_{\mathrm{yy}}$ are the horizontal normal stresses, and $\tau_{\mathrm{zz}}$ is the vertical normal stress.

A third general assumption is that the horizontal gradients of the vertical shear stresses are small compared to the vertical gradient of the vertical normal stress. If the

2, 81-158, 2009

A numerical ice sheet

- ice shelf 3D

velocity field solution

T. J. Reerink et al.

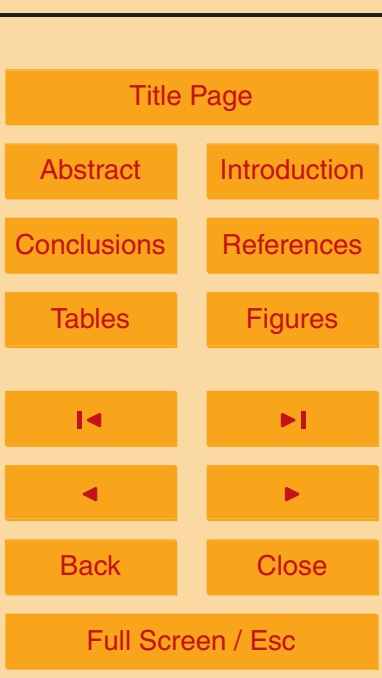

Printer-friendly Version

Interactive Discussion 
vertical stesses are relative important, e.g. in the transition zone, they are two orders smaller (Herterich, 1987; Pattyn, 2000, 2003).

$\partial_{z} \tau_{\mathrm{zz}} \gg \partial_{x} \tau_{\mathrm{zx}}, \partial_{y} \tau_{\mathrm{zy}}$

which is equivalent to the assumption that normal stress equals the weight of the layers 5 above: the hydrostatic approximation in the vertical. In contrast to a full-Stokes solution we refer to an 'almost-full-stress' solution as we take all stresses into account except those which vanish with this hydrostatic approximation. Using this third assumption (3.11) Eq. (3.10) becomes

$$
\partial_{z} \tau_{\mathrm{zz}}=\rho_{\text {ice }} g
$$

10 This equation can be integrated from layer $z$ to the surface height $H_{s}$ to obtain the vertical normal stress $\tau_{\mathrm{zz}}$ at level $z$. After this integration we have

$\tau_{\mathrm{zz}}(z)=-\rho_{\text {ice }} g\left(H_{s}-z\right)+\tau_{\mathrm{zz}}\left(H_{s}\right)$

Neglecting the vertical normal stress at the surface of 1 atmosphere means $\tau_{\mathrm{zz}}\left(H_{s}\right) \simeq 0$ which is the fourth general assumption, and leads to

${ }_{15} \tau_{\mathrm{zz}}(z)=-\rho_{\text {ice }} g\left(H_{s}-z\right)$

Substituting some linear combinations of the equation which defines the deviatoric stress tensor $\tau_{\mathrm{ij}}$ (see Eq. (A2)) into Eqs. (3.8-3.9), using Eq. (3.14) and the symmetry of the stress tensor, the set equations is expressed in deviatoric stresses (for the derivation see Appendix A4):

$$
\begin{aligned}
\partial_{z} \tau_{\mathrm{xz}}^{\prime} & =\rho_{\mathrm{ice}} g \partial_{x} H_{s}-2 \partial_{x} \tau_{\mathrm{xx}}^{\prime}-\partial_{x} \tau_{\mathrm{yy}}^{\prime}-\partial_{y} \tau_{\mathrm{xy}}^{\prime} \\
\partial_{z} \tau_{\mathrm{yz}}^{\prime} & =\rho_{\mathrm{ice}} g \partial_{y} H_{s}-2 \partial_{y} \tau_{\mathrm{yy}}^{\prime}-\partial_{y} \tau_{\mathrm{xx}}^{\prime}-\partial_{x} \tau_{\mathrm{xy}}^{\prime} \\
\tau_{\mathrm{zz}}^{\prime} & =-\tau_{\mathrm{xx}}^{\prime}-\tau_{\mathrm{yy}}^{\prime}
\end{aligned}
$$

Here, as a third equation the incompressibility condition (see Eq. (A5)) has been added. The incompressibility of ice is the fifth general assumption, and is however required deriving the constitutive relation of ice in the next subsection.

\section{GMDD}

2, 81-158, 2009

A numerical ice sheet - ice shelf 3D velocity field solution

T. J. Reerink et al.

Title Page

Abstract Introduction

Conclusions

Tables

References

Figures

14

$\rightarrow 1$

4

Back

Close

\section{Full Screen / Esc}

Printer-friendly Version

Interactive Discussion 


\subsection{Strain rate and the constitutive relation}

As the stress tensor contains the applied forces in all directions, the strain tensor contains the deformation in all directions. In fact the time derivative of the strain, the strain rate, and thus the strain rate tensor $\dot{\epsilon}_{\mathrm{ij}}$ is used, which is defined by

${ }_{5} \quad \dot{\epsilon}_{\mathrm{ij}}=\frac{1}{2}\left(\partial_{\mathrm{j}} v_{\mathrm{i}}+\partial_{\mathrm{i}} v_{\mathrm{j}}\right)$

where $v$ is the deformational velocity due to the applied stress.

The empirical based constitutive relation for ice, the ice flow law known as Glen's flow law (Paterson, 1994), relates the deviatoric stress to the strain rate by

$\tau_{\mathrm{ij}}^{\prime}=A_{\mathrm{flow}}{ }^{\frac{-1}{n}}\left(\dot{\epsilon}_{e}^{2}+\dot{\epsilon}_{0}^{2}\right)^{\frac{1-n}{2 n}} \dot{\epsilon}_{\mathrm{ij}}$

10 where $\dot{\epsilon}_{0}^{2}=10^{-30}$ is added to prevent dividing by zero in numerical evaluations, and with

$\dot{\epsilon}_{e}^{2} \equiv \dot{\epsilon}_{\mathrm{xx}}{ }^{2}+\dot{\epsilon}_{\mathrm{yy}}{ }^{2}+\dot{\epsilon}_{\mathrm{xx}} \dot{\epsilon}_{\mathrm{yy}}+\dot{\epsilon}_{\mathrm{xy}}{ }^{2}+\dot{\epsilon}_{\mathrm{xz}}{ }^{2}+\dot{\epsilon}_{\mathrm{yz}}{ }^{2}$

being minus the second invariant of $\dot{\epsilon}$.

\subsection{The general 3D velocity field}

To obtain the velocity equations the flow law for ice is substituted in the momentum equations. Recalling Eq. (3.19), substituting definition (3.20), and thereafter the substitution of Eq. (3.18) leads to

$$
\begin{aligned}
\tau_{\mathrm{ij}}^{\prime} & =A_{\mathrm{flow}}{ }^{\frac{-1}{n}}\left(\dot{\epsilon}_{e}^{2}+\dot{\epsilon}_{0}^{2}\right)^{\frac{1-n}{2 n}} \dot{\epsilon}_{\mathrm{ij}} \\
& =A_{\text {flow }}{ }^{\frac{-1}{n}}\left(\dot{\epsilon}_{\mathrm{xx}}^{2}+\dot{\epsilon}_{\mathrm{yy}}^{2}+\dot{\epsilon}_{\mathrm{xx}} \dot{\epsilon}_{\mathrm{yy}}+\dot{\epsilon}_{\mathrm{xy}}^{2}+\dot{\epsilon}_{\mathrm{xz}}{ }^{2}+\dot{\epsilon}_{\mathrm{yz}}{ }^{2}+\dot{\epsilon}_{0}^{2}\right)^{\frac{1-n}{2 n}} \dot{\epsilon}_{\mathrm{ij}} \\
& =\frac{1}{2} C^{\mathrm{uvw}}\left(\partial_{\mathrm{j}} v_{\mathrm{i}}+\partial_{\mathrm{i}} v_{\mathrm{j}}\right)
\end{aligned}
$$

\section{GMDD}

2, 81-158, 2009

A numerical ice sheet - ice shelf 3D velocity field solution

T. J. Reerink et al.

Title Page

Abstract

Introduction

Conclusions

Tables

References

Figures

14

$\rightarrow$

4

Back

Close 
In which $C^{\mathrm{uvw}}$ abbreviates the fixed components. In that sense 'fixed', that they don't depend on the deviatoric stress indices $\mathrm{i}$ and j. $C^{\mathrm{uvw}}$ is defined by

\section{GMDD}

$$
\begin{aligned}
C^{\mathrm{uvw}} \equiv & A_{\mathrm{flow}} \frac{-1}{n}\left(\left(\partial_{x} u\right)^{2}+\left(\partial_{y} v\right)^{2}+\left(\partial_{x} u\right)\left(\partial_{y} v\right)\right. \\
& \left.+\frac{1}{4}\left[\left(\partial_{y} u+\partial_{x} v\right)^{2}+\left(\partial_{z} u+\partial_{x} w\right)^{2}+\left(\partial_{z} v+\partial_{y} w\right)^{2}\right]+\dot{\epsilon}_{0}^{2}\right)^{\frac{1-n}{2 n}}
\end{aligned}
$$

5 In Eq. (3.22) each component is expressed in $u, v$, and $w$. Substituting Eq. (3.22) in the set of Eqs. (3.15-3.17) results in a set of equations expressed entirely in $u, v$, and w

$$
\begin{gathered}
\rho_{\text {ice }} g \partial_{x} H_{s}=\frac{1}{2} \partial_{z}\left(C^{\mathrm{uvw}}\left(\partial_{z} u+\partial_{x} w\right)\right)+2 \partial_{x}\left(C^{\mathrm{uvw}} \partial_{x} u\right)+\partial_{x}\left(C^{\mathrm{uvw}} \partial_{y} v\right) \\
+\frac{1}{2} \partial_{y}\left(C^{\mathrm{uvw}}\left(\partial_{y} u+\partial_{x} v\right)\right)
\end{gathered}
$$

10 $\rho_{\text {ice }} g \partial_{y} H_{s}=\frac{1}{2} \partial_{z}\left(C^{\mathrm{uvw}}\left(\partial_{z} v+\partial_{y} w\right)\right)+2 \partial_{y}\left(C^{\mathrm{uvw}} \partial_{y} v\right)+\partial_{y}\left(C^{\mathrm{uvw}} \partial_{x} u\right)$

$$
\begin{aligned}
& +\frac{1}{2} \partial_{x}\left(C^{\mathrm{uvw}}\left(\partial_{y} u+\partial_{x} v\right)\right) \\
& 0=\partial_{x} u+\partial_{y} v+\partial_{z} w
\end{aligned}
$$

This set of three elliptical second-order partial differential equations with three unknowns $u, v$, and $w$, has to be solved coupled to obtain an almost-full-stress solution.

\subsection{The 3D boundary conditions}

To solve the resulting elliptical set of partial differential equations numerically, we need several boundary conditions. At the horizontal domain boundaries of the grid a smooth extension is assumed for all three velocity components by assuming:

$\partial_{x} u=\partial_{x} v=\partial_{x} w=0$

A numerical ice sheet - ice shelf 3D velocity field solution

T. J. Reerink et al.

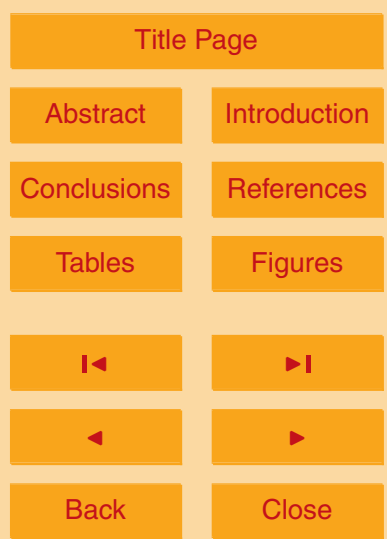

Full Screen / Esc

Printer-friendly Version

Interactive Discussion 
in the $x$-direction. And in the y-direction by

$\partial_{y} u=\partial_{y} v=\partial_{y} w=0$

This is a slightly easier approach than applying a stress free condition, as e.g. derived by Deponti et al. (2006), but without a significant difference. The atmosphere-ice in5 terface and the ice-water interface at the bottom of the shelf are both taken as free surfaces by imposing:

$\partial_{z} u=\partial_{z} v=\partial_{z} w=0$

At the bottom of the sheet the horizontal velocity components are prescribed with Dirichlet conditions. For large parts of the sheet this prescribed bottom horizontal 10 velocities will be zero, representing a situation in which the ice is frozen to the bedrock. For other parts of the sheet like for ice-stream areas in particular, this prescribed velocities will be the sliding velocities:

$u=u_{\text {sliding }}$

$v=v_{\text {sliding }}$

15 The vertical velocity at the bottom follows from a kinematic condition and is applied as a Dirichlet condition, with the boundary condition at the ice-bedrock interface given by

$w\left(H_{b}\right)=\partial_{t} H_{b}+M_{b}+u\left(H_{b}\right) \partial_{x} H_{b}+v\left(H_{b}\right) \partial_{y} H_{b}$

where $w\left(H_{b}\right)$ is the vertical velocity at the ice-bedrock interface and $u\left(H_{b}\right)$ and $v\left(H_{b}\right)$ are the sliding velocities at the ice-bedrock interface. Note that the vertical movement of the bedrock $\partial_{t} H_{b}$ might differ from $w\left(H_{b}\right)$ due to the basal melt and the advection in the lowest ice layer.

GMDD

2, 81-158, 2009

A numerical ice sheet - ice shelf 3D velocity field solution

T. J. Reerink et al.

Title Page

Abstract

Introduction

Conclusions

Tables

References

Figures

14

$>1$

4

Back

Close

Full Screen / Esc

Printer-friendly Version

Interactive Discussion 


\section{A formulation of the general velocity equations facilitating several stress ap- proximations}

In the previous section the momentum Eqs. (3.15-3.17) are combined with the flow-law Eq. (3.22) and expressed in $u, v$, and $w$ (Eqs. (3.23-3.26)). The two reasons to express 5 those equations in $u, v$, and $w$ are: Firstly, substituting three independent variants of Eq. (3.22) in Eqs. (3.15-3.17) yields a closed set, leaving three unknowns in the three Eqs. (3.24-3.26). Secondly, the boundary conditions at the ice-bedrock interface, such as the parameterized sliding velocity, the zero velocities in case of a frozen situation, and $w\left(H_{b}\right)$ as in Eq. (3.32), are expressed in terms of velocities instead of stresses. The velocity representation conveniently matches both cases.

However, in the velocity representation it is difficult to see from which stress terms the velocity terms originate. Thus, attaining the stress approximations becomes cumbersome. Therefore, in this section we construct, by intoducing seven simplification coefficients $f_{0}-f_{6}$, a differential representation of the velocity field which allows straightforward stress approximations. These simplification coefficients have values 0 or 1 for terms being omitted or taken into account respectively (see also Table 2). Including them we can rewrite the set of Eqs. (3.24-3.26) to

$$
\begin{aligned}
\rho_{\text {ice }} g \partial_{x} H_{s}= & \frac{1}{2} f_{4} \partial_{z}\left(C^{\mathrm{uvw}}\left(\partial_{z} u+f_{0} \partial_{x} w\right)\right)+2 f_{5} \partial_{x}\left(C^{\mathrm{uvw}} \partial_{x} u\right)+f_{5} \partial_{x}\left(C^{\mathrm{uvw}} \partial_{y} v\right) \\
& +\frac{1}{2} f_{6} \partial_{y}\left(C^{\mathrm{uvw}}\left(\partial_{y} u+\partial_{x} v\right)\right) \\
20 \rho_{\mathrm{ice}} g \partial_{y} H_{s}= & \frac{1}{2} f_{4} \partial_{z}\left(C^{\mathrm{uvw}}\left(\partial_{z} v+f_{0} \partial_{y} w\right)\right)+2 f_{5} \partial_{y}\left(C^{\mathrm{uvw}} \partial_{y} v\right)+f_{5} \partial_{y}\left(C^{\mathrm{uvw}} \partial_{x} u\right) \\
& +\frac{1}{2} f_{6} \partial_{x}\left(C^{\mathrm{uvw}}\left(\partial_{y} u+\partial_{x} v\right)\right) \\
0= & \partial_{x} u+\partial_{y} v+\partial_{z} w
\end{aligned}
$$

A numerical ice sheet - ice shelf 3D velocity field solution

T. J. Reerink et al.

\section{Title Page}

Abstract Introduction

Conclusions

Tables

References

Figures

14

-1

4

Back

Close

\section{Full Screen / Esc}


and we redefine Eq. (3.23) by

$$
\begin{aligned}
C^{\mathrm{uvw}} \equiv & A_{\text {flow }} \frac{-1}{n}\left(f_{1}\left(\partial_{x} u\right)^{2}+f_{1}\left(\partial_{y} v\right)^{2}+f_{1}\left(\partial_{x} u\right)\left(\partial_{y} v\right)\right. \\
& \left.+\frac{1}{4}\left[f_{2}\left(\partial_{y} u+\partial_{x} v\right)^{2}+f_{3}\left(\partial_{z} u+f_{0} \partial_{x} w\right)^{2}+f_{3}\left(\partial_{z} v+f_{0} \partial_{y} w\right)^{2}\right]+\dot{\epsilon}_{0}^{2}\right)^{\frac{1-n}{2 n}}
\end{aligned}
$$

Most models use another (sixth) general assumption

$5 \partial_{z} u \gg \partial_{x} w$

$\partial_{z} v \gg \partial_{y} w$

This assumption can be applied by taking $f_{0}=0$. In that case the first two equations of this set depend only on $u$ and $v$. After solving $u$ and $v$ coupled, $w$ can solved with Eq. (4.3) separately. The longitudinal stress, the side shear and the horizontal 10 shear can be neglected by taking respectively $f_{1}, f_{2}$ or $f_{3}$ zero. Several derivatives, as presented in Table 2 can be neglected separately by taking $f_{4}, f_{5}$ or $f_{6}$ zero.

The almost-full-stress solution is suitable for solving the combined velocity field for the sheet, the shelf and the transition zone. Because of the simplification coefficients it is possible to use this set in case of different stress approximations for the sheet and 15 the shelf. In both cases the advantage of using a single set of equations for the sheet and the shelf is that no interior boundary has to be handled.

\subsection{Usual shelf and sheet approximations}

If we take the usual shelf approximations we have $f_{0}=f_{3}=f_{4}=0$ and $f_{1}=f_{2}=f_{5}=f_{6}=1$, as in Table 2. Substituting these values in Eqs. (4.1-4.4), we obtain

A numerical ice sheet - ice shelf 3D velocity field solution

T. J. Reerink et al.

Title Page

Abstract

Introduction

Conclusions

Tables

References

Figures

14

$\rightarrow$

4

Back

Close

Full Screen / Esc

Printer-friendly Version

Interactive Discussion

$$
\begin{aligned}
\rho_{\text {ice }} g \partial_{y} H_{s} & =2 \partial_{y}\left(C^{\mathrm{uvw}} \partial_{y} v\right)+\partial_{y}\left(C^{\mathrm{uvw}} \partial_{x} u\right)+\frac{1}{2} \partial_{x}\left(C^{\mathrm{uvw}}\left(\partial_{y} u+\partial_{x} v\right)\right) \\
0 & =\partial_{x} u+\partial_{y} v+\partial_{z} w
\end{aligned}
$$


with

$C^{\mathrm{uvw}}=A_{\mathrm{flow}} \frac{-1}{n}\left(\left(\partial_{x} u\right)^{2}+\left(\partial_{y} v\right)^{2}+\left(\partial_{x} u\right)\left(\partial_{y} v\right)+\frac{1}{4}\left[\left(\partial_{y} u+\partial_{x} v\right)^{2}\right]+\dot{\epsilon}_{0}^{2}\right)^{\frac{1-n}{2 n}}$

resembling the shelf equations in Huybrechts (1991). The $u$ and $v$ component have to be solved coupled in these shelf Eqs. (4.7-4.8), while the uncoupled $w$ can be 5 calculated with Eq. (4.9) afterwards.

For the sheet the usual approximations $(S I A)$ are $f_{0}=f_{1}=f_{2}=f_{5}=f_{6}=0$ and $f_{3}=f_{4}=1$, as in Table 2. Equations (4.1-4.4) reduces with these values to

$\rho_{\text {ice }} g \partial_{x} H_{s}=\frac{1}{2} \partial_{z}\left(C^{\mathrm{uvw}} \partial_{z} u\right)$

$\rho_{\text {ice }} g \partial_{y} H_{s}=\frac{1}{2} \partial_{z}\left(C^{\mathrm{uvw}} \partial_{z} v\right)$

$$
0=\partial_{x} u+\partial_{y} v+\partial_{z} w
$$

with

$C^{\mathrm{uvw}}=A_{\mathrm{flow}} \frac{-1}{n}\left(\frac{1}{4}\left[\partial_{z} u^{2}+\partial_{z} v^{2}\right]+\dot{\epsilon}_{0}^{2}\right)^{\frac{1-n}{2 n}}$

which is the differential representation. Usual for the SIA the integral representation is taken, following a slightly different approach. The $u$ and $v$ component can be solved by Eqs. (4.11-4.12) respectively, and $w$ can be calculated with Eq. (4.13) afterwards.

\section{The numerical scheme for the almost-full-stress velocity solution}

In this section and in the accompanying appendices we show the transformation of the set of Eqs. (4.1-4.3) to the coordinates $x, y$, and $\zeta$, with $\zeta$ the non-equidistant vertically scaled coordinate (see Fig. 2). Subsequently, we demonstrate the discretization of that result. And finally, we show how to put it in a huge sparse matrix and how to solve these three equations with three coupled unknowns simultaneously on all grid cells.

A numerical ice sheet - ice shelf 3D velocity field solution

T. J. Reerink et al.

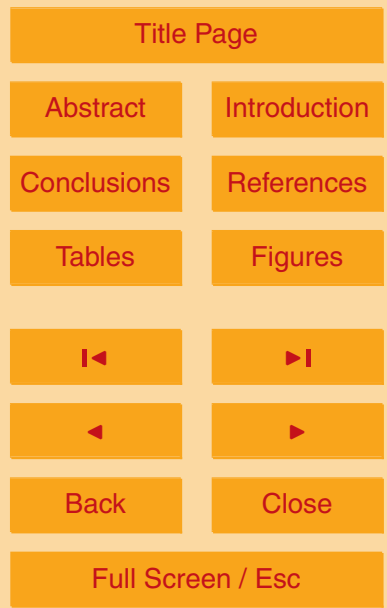

Printer-friendly Version

Interactive Discussion 
A step by step derivation is given in Appendix $D$, to which we will refer here several times. In Appendix $E$ the structure of the matrix equation and a compact format for such a sparse matrix is given and derived.

GMDD

2, 81-158, 2009

5.1 Index-derivative notation

5 Working out the derivatives in Eqs. (4.1-4.3), recombining the resulting terms, and changing to the index-derivative notation (e.g. $W_{x}=\partial_{x} w, U_{y y}=\partial_{y y}^{2} u, C_{z}^{u v w}=\partial_{z} C^{u v w}$, etc.), we get as in Eqs. (D10-D12) in Appendix D:

$$
\begin{aligned}
\frac{2 \rho_{\text {ice }} g}{C^{\mathrm{uvw}}} \partial_{x} H_{s} & =f_{4} U_{z z}+f_{0} f_{4} w_{z x}+4 f_{5} U_{x x}+f_{6} U_{y y}+f_{56} V_{x y} \\
& +\frac{1}{C^{\mathrm{uvw}}}\left[f_{4} C_{z}^{u v w}\left(U_{z}+f_{0} W_{x}\right)+f_{5} C_{x}^{u v w}\left(4 U_{x}+2 V_{y}\right)+f_{6} C_{y}^{u v w}\left(U_{y}+V_{x}\right)\right]
\end{aligned}
$$

$$
\begin{aligned}
\frac{2 \rho_{\text {ice }} g}{C^{\mathrm{uvw}} \partial_{y} H_{s}} & =f_{4} V_{z z}+f_{0} f_{4} W_{z y}+4 f_{5} V_{y y}+f_{6} V_{x x}+f_{56} U_{x y} \\
& +\frac{1}{C^{\mathrm{uvw}}}\left[f_{4} C_{z}^{u v w}\left(V_{z}+f_{0} W_{y}\right)+f_{5} C_{y}^{u v w}\left(4 V_{y}+2 U_{x}\right)+f_{6} C_{x}^{u v w}\left(U_{y}+V_{x}\right)\right] \\
0 & =U_{x}+V_{y}+W_{z}
\end{aligned}
$$

$f_{56} \equiv 2 f_{5}+f_{6}$

5.2 Transformation towards the scaled coordinates

This set of equations has to be transformed to the scaled coordinates $x, y$, and $\zeta$. The related transformation rules for the derivative operators, generating correction terms for $x, y$, and $t$ derivatives because the scaling is done per grid point and per time step, are presented in Appendix B and are summarized in Table 3. Below the accents for the
A numerical ice sheet - ice shelf 3D velocity field solution

T. J. Reerink et al.

Title Page

Abstract

Introduction

Conclusions

Tables

References

Figures

14

$\rightarrow 1$

4

Back

Close

Full Screen / Esc

Printer-friendly Version

Interactive Discussion 
new $x$ and $y$ coordinates are omitted. Rearranging the terms by the derivatives of $u, v$, and $w$, and defining new coefficients in front of those derivative terms, the transformed equations become (as in Eqs. (D24-D26) in Appendix D):

$f_{5}^{U_{x}} U_{x}+f_{6}^{U_{y}} U_{y}+f_{456}^{U_{\zeta}} U_{\zeta}+f_{5}^{U_{x x}} U_{x x}+f_{5}^{U_{x \zeta}} U_{x \zeta}+f_{6}^{U_{y y}} U_{y y}+f_{6}^{U_{y \zeta}} U_{y \zeta}+f_{456}^{U_{\zeta \zeta}} U_{\zeta \zeta}+$

${ }_{5} f_{6}^{V_{x}} V_{x}+f_{5}^{V_{y}} V_{y}+f_{56}^{V_{\zeta}} V_{\zeta}+f_{56}^{V_{x y}} V_{x y}+f_{56}^{V_{x \zeta}} V_{x \zeta}+f_{56}^{V_{y \zeta}} V_{y \zeta}+f_{56}^{V_{\zeta \zeta}} V_{\zeta \zeta}+$

$f_{04}^{W_{x}} W_{x}+f_{04}^{W_{\zeta}} W_{\zeta}+f_{04}^{W_{x \zeta}} W_{x \zeta}+f_{04}^{W_{\zeta \zeta}} W_{\zeta \zeta}=\frac{2 \rho_{\text {ice }} g}{c^{\text {uvw }}} \partial_{x} H_{s}$

$g_{5}^{U_{x}} U_{x}+g_{6}^{U_{y}} U_{y}+g_{56}^{U_{\zeta}} U_{\zeta}+g_{56}^{U_{x y}} U_{x y}+g_{56}^{U_{x \zeta}} U_{x \zeta}+g_{56}^{U_{y \zeta}} U_{y \zeta}+g_{56}^{U_{\zeta \zeta}} U_{\zeta \zeta}+$

$g_{6}^{V_{x}} V_{x}+g_{5}^{V_{y}} V_{y}+g_{456}^{V_{\zeta}} V_{\zeta}+g_{6}^{V_{x x}} V_{x x}+g_{6}^{V_{x \zeta}} V_{x \zeta}+g_{5}^{V_{y y}} V_{y y}+g_{5}^{V_{y \zeta}} V_{y \zeta}+g_{456}^{V_{\zeta \zeta}} V_{\zeta \zeta}+$

$g_{04}^{W_{y}} W_{y}+g_{04}^{W_{\zeta}} W_{\zeta}+g_{04}^{W_{y \zeta}} W_{y \zeta}+g_{04}^{W_{\zeta \zeta}} W_{\zeta \zeta}=\frac{2 \rho_{\text {ice }} g}{C^{\mathrm{uvw}}} \partial_{y} H_{s}$

$U_{x}+\zeta_{x} U_{\zeta}+V_{y}+\zeta_{y} V_{\zeta}+\zeta_{z} W_{\zeta}=0$

The coefficients are defined in Table 5. The transformed derivative fields from $C^{\mathrm{uvw}}$ devided by $C^{\mathrm{uvw}}$, are defined as in Eqs. (D17-D19) in Appendix D, by

$c^{x}=\frac{C_{x}^{u v w}+\zeta_{x} C_{\zeta}^{u v w}}{C^{u v w}}$

$C^{y}=\frac{C_{y}^{u v w}+\zeta_{y} C_{\zeta}^{u v w}}{C^{u v w}}$

$C^{\zeta}=\frac{\zeta_{z} C_{\zeta}^{u v w}}{C^{u v w}}$
GMDD

2, 81-158, 2009

A numerical ice sheet

- ice shelf 3D

velocity field solution

T. J. Reerink et al.

Title Page

Abstract

Introduction

Conclusions

References

Tables

Figures

14

$\rightarrow 1$

4

Back

Close

Full Screen / Esc

Printer-friendly Version 
these quantities can be calculated explicitly by taking field derivatives of $C^{\mathrm{uvw}} \cdot C^{\mathrm{uvw}}$ has to be expressed in $\zeta$ coordinates as in Eq. (D20), like

\section{GMDD}

$$
\begin{aligned}
& C^{\mathrm{uvw}}(x, y, \zeta)=A_{\mathrm{flow}}{ }^{\frac{-1}{n}}\left(f_{1}\left(\partial_{x} u+\zeta_{x} \partial_{\zeta} u\right)^{2}+f_{1}\left(\partial_{y} v+\zeta_{y} \partial_{\zeta} v\right)^{2}+\right. \\
& f_{1}\left(\partial_{x} u+\zeta_{x} \partial_{\zeta} u\right)\left(\partial_{y} v+\zeta_{y} \partial_{\zeta} v\right)+ \\
& \frac{1}{4}\left[f_{2}\left(\partial_{y} u+\zeta_{y} \partial_{\zeta} u+\partial_{x} v+\zeta_{x} \partial_{\zeta} v\right)^{2}+\right. \\
& f_{3}\left(\zeta_{z} \partial_{\zeta} u+f_{0}\left(\partial_{x} w+\zeta_{x} \partial_{\zeta} w\right)\right)^{2}+ \\
&\left.\left.f_{3}\left(\zeta_{z} \partial_{\zeta} v+f_{0}\left(\partial_{y} w+\zeta_{y} \partial_{\zeta} w\right)\right)^{2}\right]+\dot{\epsilon}_{0}^{2}\right)^{\frac{1-n}{2 n}}
\end{aligned}
$$

In which $A_{\text {flow }}$ depends on $T(x, y, \zeta)$, the spatial dependent ice temperature.

The boundary conditions in the $x$-direction in Eqs. (3.27-3.29) become transformed

$10 \partial_{x} u+\zeta_{x} \partial_{\zeta} u=\partial_{x} v+\zeta_{x} \partial_{\zeta} v=\partial_{x} w+\zeta_{x} \partial_{\zeta} w=0$

and in the $y$-direction

$\partial_{y} u+\zeta_{\mathrm{y}} \partial_{\zeta} u=\partial_{y} v+\zeta_{\mathrm{y}} \partial_{\zeta} v=\partial_{y} w+\zeta_{\mathrm{y}} \partial_{\zeta} w=0$

while for the atmosphere-ice interface and the ice-water interface the transformed boundary conditions become

$\partial_{z} u=\partial_{z} v=\partial_{z} w=0$ for $\zeta_{z} \neq 0$

\subsection{Discretization}

The transformed set of Eqs. (5.5-5.7) can be discretized in several ways. In the approach here all the linear terms are taken implicit, while the non-linear part $C^{\text {uvw }}$ is taken explicit. We discretize the linear velocity components, with use of the finite difference rules from Table 3. After rearranging the terms and defining another amount of convenient coefficients (see Tables 6-8) we obtain the final discretized equations which are presented in Appendix D in the Eqs. (D30-D32). 
We take from Appendix E Eq. (E25):

$$
\left(a_{s}^{\prime}(i j k)\left[c_{1}, c_{m}+r_{s}^{\prime}\right]\right)\left(v_{1}(i j k)\left[c_{1}\right]\right)=\left(r h s^{\prime}(i j k)\left[c_{1}\right]\right)
$$

The coefficients in matrix $A$ are denoted by $a$ and are labeled with a superscript which 5 refers to the related equation number (between 1 and $n$ ). The 'sparse' subscript $s$ lists for each /-th equation all the $q$, non-zero matrix coefficients. The $i j k$ between parenthesis denotes the dependence on the different grid points of each matrix coefficient. And between brackets the row and column indices in the matrix equation are given with help of some grid dependent central indices $c_{/}(i j k)$, see Eqs. (E13-E14). The 10 structure of matrix Eq. (5.15) is explained in Appendix E. In this appendix also is explained how to interpret the notation concerning the ranges of several indices. For the I-th equation there are $q$, sparse (non-zero) matrix coefficients and an equal amount of relative column indices:

$a_{1}^{\prime} \leq a_{s}^{\prime} \leq a_{q_{1}}^{\prime}$

$r_{1}^{\prime} \leq r_{s}^{\prime} \leq r_{a_{1}}^{\prime}$

with, in our case of a three-dimensional grid $(n=3)$, the indices $I, q_{l}$, and $s$ in the range:

$$
\begin{aligned}
1 & \leq l \leq 3 \\
q_{1} & \leq q_{1} \leq q_{3} \\
1 & \leq s \leq q_{1}
\end{aligned}
$$

2, 81-158, 2009

A numerical ice sheet - ice shelf 3D velocity field solution

T. J. Reerink et al.

20 with in our case $q_{1}=q_{2}=43$ and $q_{3}=13$. Knowing their positions we can construct the three arrays containing the relative column indices: $\left\{r_{1}^{1}, \ldots, r_{43}^{1}\right\},\left\{r_{1}^{2}, \ldots, r_{43}^{2}\right\}$, 
$\left\{r_{1}^{3}, \ldots, r_{13}^{3}\right\}$. With $v_{1}=u, v_{2}=v, v_{3}=w$. Writing out Eq. (5.15) for $I=\{1,2,3\}$ we have:

$$
\left(\begin{array}{l}
a_{1}^{1}\left[c_{1}, c_{m}+r_{1}^{1}\right] \ldots a_{43}^{1}\left[c_{1}, c_{m}+r_{43}^{1}\right] \\
a_{1}^{2}\left[c_{2}, c_{m}+r_{1}^{2}\right] \ldots a_{43}^{2}\left[c_{2}, c_{m}+r_{43}^{2}\right] \\
a_{1}^{3}\left[c_{3}, c_{m}+r_{1}^{n}\right] \ldots a_{13}^{3}\left[c_{3}, c_{m}+r_{13}^{n}\right]
\end{array}\right)\left(\begin{array}{l}
v_{1}\left[c_{1}\right] \\
v_{2}\left[c_{2}\right] \\
v_{3}\left[c_{3}\right]
\end{array}\right)=\left(\begin{array}{l}
r h s^{1}\left[c_{1}\right] \\
r h s^{2}\left[c_{2}\right] \\
r h s^{3}\left[c_{3}\right]
\end{array}\right)
$$

where the dependency of the components and of the central indices on $i j k$ is omitted, to keep the notation short. Note that the sparse matrix coefficients in Eq. (5.21) not 5 necessary occur in the same columns.

\subsection{Solving}

The non-zero coefficients $a_{s}^{\prime}(i j k)\left[c_{l}, c_{m}+r_{s}^{l}\right]$ standing ahead of the discretized variables in the discretized Eqs. (D30-D32), are defined in Tables 6-8 for the first, the second, and the third equation respectively. The name-position rule (E15) relates the name of each matrix coefficient with its position in the matrix:

$a_{\left(v_{m}\right)_{i+d_{i}, j+d_{j}}^{k+d_{k}}}^{\prime}\left[c_{1}, c_{m}+d_{i} e_{i}+d_{j} e_{j}+d_{k} e_{k}\right]$

The non-zero coefficients can be stored in a certain order of $i, j, k, l$ and $s$ in a vector $A_{s}$. While the row indices, the column indices, and the right hand sides will be stored in this same order in the vectors $B_{s}, C_{s}$, and $R H S$ respectively.

15 The domain boundary conditions for the first, the second, and the third equation are given in Table 9. The boundary conditions applied to the horizontal outer planes of the computational grid are physical conditions. At the bottom of the sheet (the ice-bedrock interface), the sliding velocities and $w\left(H_{b}\right)$ as in Eq. (3.32) are applied as Dirichlet conditions. The surface (the atmosphere-ice interface) and the shelf bottom (the ice-

water interface) are taken as free surfaces by applying Neumann conditions. Assuming a smooth extension beyond the horizontal domain boundaries, we also apply Neumann conditions at the vertical side planes of the computational grid.

\section{GMDD}

2, 81-158, 2009

A numerical ice sheet - ice shelf 3D velocity field solution

T. J. Reerink et al.

Title Page

Abstract Introduction

Conclusions

Tables

References

Figures

14

$\rightarrow 1$

4

Back

Close 
Because $A_{s}, B_{s}$ and $C_{s}$ contain the sparse matrix efficiently, we call this the compact three vector format of $A$. These four vectors $A_{s}, B_{s}, C_{s}$, and $R H S$ form the common format of input for a sparse matrix solver. With this input the 3D 3-component velocity field with three coupled equations can be solved.

\section{Discussion}

Taking five assumptions which are valid for both the sheet and the shelf, we derived a general set of velocity equations for the combined sheet-shelf system. The dominance of shear in large parts of the sheet, and compared to that its absence in the shelf, does not allow other general assumptions. With the introduction of the 'simplification coefficients' in Eqs. (4.1-4.3), several stress approximations are represented in the velocity representation. The familiar subsets of equations for the sheet and the shelf are immediately returned by substituting the combination of simplification coefficients as presented in Table 2.

These simplification coefficients, which are easy to implement in a code, are not more than a tool to investigate the stress approximations themselves. Adjusting some of the simplification coefficients to zero is equivalent to applying a certain stress approximation. With this set up and without an internal boundary at the grounding line, one single code can handle the almost-full-stress approach, but also several different approximation cases. This prevents the interference in result-differences due to specific approximation codes.

Solving the general set of coupled velocity Eqs. (4.1-4.3) has the advantage that:

- The internal boundary problem between the sheet and the shelf disappears. Usually this internal boundary coincides with the grounding line of which the pattern is irregular and varies in time. Our approach avoids handling such a complicated internal boundary with an additional specific stress treatment at the grounding line. The latter is possible, due to the fact that the longitudinal stresses are included for the entire sheet-shelf system.

A numerical ice sheet - ice shelf 3D velocity field solution

T. J. Reerink et al.

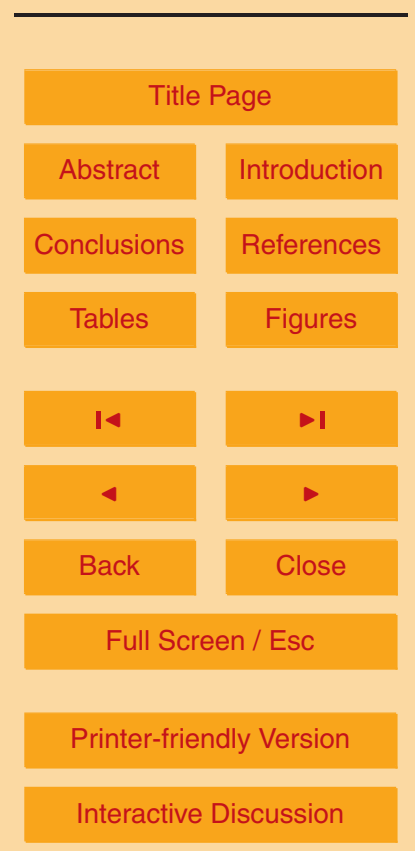

Interactive Discussion 
- The transition zone and the ice divide are both treated accurate because the longitudinal stresses are included for both the sheet and the shelf. Lateral shear working on shelves is included as well.

- It enables testing with the simplification coefficients as described above.

- Within the shelf a temperature dependent 3D velocity field is calculated.

Although in our methodology the grounding line needs no special treatment anymore, it might still be necessary to use a finer grid near the grounding line to obtain accurate grounding line migration.

The final general velocity solution clearly shows that there are only two differences between the sheet and the shelf:

- The boundary conditions at the ice bottom differ, see Table 9. For the shelf this is an ice-water interface where friction is neglected. For the sheet, on the other hand, this is an ice-bedrock interface where friction depends on a temperature dependent threshold for sliding.

15 - The way of calculating the surface heigth $H_{s}$, see Eq. (3.1). The the condition in this equation distinguishes between the sheet and the shelf.

Our set of three coupled velocity Eqs. (4.1-4.3) can be solved numerically in several ways. However, certain choices lead to interference of complexity. The vertically scaled coordinate, often used in ice dynamics, has considerable advantages. Noting that both surface and bottom levels vary at each grid point, the boundary conditions of the numerical ice grid are easy to handle. In addition, this vertical scaling enables the use of a large range of different vertical grid distances necessary to adequately describe the wide range of ice-cap geometries which also vary widely different over time. Furthermore, a non-equidistant scaling allows a more accurate description of the processes at the bottom of the sheet. With an addapting bedrock level due to changes in the ice load history, the vertically scaled ice coordinate is convenient too because

\section{GMDD}

2, 81-158, 2009

A numerical ice sheet - ice shelf 3D velocity field solution

T. J. Reerink et al.

Title Page

Abstract

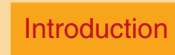

Conclusions

Tables

References

Figures

14

$>1$

4

Back

Close

Full Screen / Esc

Printer-friendly Version

Interactive Discussion 
it directly connects in the vertical without any gap between the bedrock and the ice. However, a disadvantage is that this scaling introduces extra terms in the derivative operators. Thus, because we have many terms with double and mixed derivatives, the vertically scaled Eqs. (5.5-5.7) even contain a larger number of terms.

$5 \quad$ To solve this vertically scaled set of coupled elliptical differential velocity equations numerically, a decision has to be made on the implicit/explicit treatment of the different terms. In that perspective we choose the most complicated approach by taking all linear terms implicit. Taking the non-linear term in Eq. (5.11) implicit is almost impossible because the non-linearity is caused by the temperature dependent ice flow parameter ${ }_{10} A_{\text {flow }}$. The temperature in this parameter depends on the 3D space and the time, and on the exponent which is a function of the flow-law exponent.

Taking all linear terms implicit for the three coupled velocity equations means that they have to be solved simultaneously on the $3 \mathrm{D}$ grid. This technique is developed in Appendix E.

15 Solving those equations on a 3D grid simultaneously, with their many subdiagonals in our matrix Eq. (5.21) caused by the many correction terms due to the vertical scaling, becomes an intensive computational job. Working memory and CPU-time complicates the practical application so far, although it is possible to deal with. A suited direct solver in such a case is MUMPS (Amestoy et al., 2000), but an iterative method using the combination of GRMS with a preconditioner from the PATSc package (Balay et al., 2004) might be favourable in such a 3D case.

However, with the insight of our complete approach it should be possible to find sufficient implicit/explicit variants which are easier to manage. E.g. with an iterating Red-Black Gauss Seidel over-relaxation method, the three space directions can be taken implicit by permutation. Or in our case if $f_{0}=0$ the third grid dimension uncouples, which reduces our matrix equations to the case of two coupled equations: $n=2$ in Appendix E.

Other implicit/explicit choices can be made, for which the consequences further on in the derivation can be followed by tracing the simplification coefficients.

GMDD

2, 81-158, 2009

A numerical ice sheet - ice shelf 3D velocity field solution

T. J. Reerink et al.

Title Page

Abstract

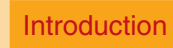

Conclusions

Tables

References

Figures

14

$\rightarrow$

4

Back

Close

Full Screen / Esc

Printer-friendly Version

Interactive Discussion 


\section{Conclusions}

We documented an integral sheet-shelf modelling approach, in which various stress approximations can be made by only adjusting some of the simplification coefficients. The general velocity equations for the combined sheet-shelf system are derived

5 straightforwardly. We showed how these partial differential equations and their boundary conditions are transformed to the locally and time wise scaled coordinates typically used in ice-cap dynamics, and how they are (non-equidistantly) discretized in case all linear terms are taken implicitly. Finally, a matrix equation format is developed which contains these coupled equations for a 3D case. Once the implicit/explicit scheme has been chosen, many numerical approaches are possible. How useful these are depends on the consistence with the prior physical assumptions. This work enables one to decide which physical assumptions and which numerical choices can be combined.

\section{Appendix A}

\section{Deviatoric stresses}

In this appendix the hydrostatic pressure, deviatoric stress, hydrostatic equilibrium, and incompressibility are defined, whereupon the stress equations for ice can be rewritten in terms of deviatoric stress.

\section{A1 The deviatoric stress tensor}

20 The hydrostatic pressure $p \delta_{\mathrm{ij}}$, having normal stress components only, has an amplitude $p$ being the mean of the normal stresses:

$p=\frac{1}{3}\left(\tau_{\mathrm{xx}}+\tau_{\mathrm{yy}}+\tau_{\mathrm{zz}}\right)=\frac{1}{3} \tau_{\mathrm{kk}}$

\section{GMDD}

2, 81-158, 2009

A numerical ice sheet - ice shelf 3D velocity field solution

T. J. Reerink et al.

\section{Title Page}

Abstract

Introduction

Conclusions

Tables

References

Figures

14

I

4

Back

Close

\section{Full Screen / Esc}

Printer-friendly Version

Interactive Discussion 
Where $\tau$ represents the full stress tensor and $k$ indicates a summation index. The deviatoric stress $\tau$ is defined as the full stress minus the hydrostatic pressure, which is in component form:

$\tau_{\mathrm{ij}}^{\prime} \equiv \tau_{\mathrm{ij}}-p \delta_{i j}=\tau_{\mathrm{ij}}-\frac{1}{3} \tau_{\mathrm{kk}} \delta_{\mathrm{ij}}$

5 A2 Hydrostatic equilibrium

In case of hydrostatic equilibrium the normal stresses are equal $\tau_{\mathrm{xx}}=\tau_{\mathrm{yy}}=\tau_{\mathrm{zz}}$, so the $x$ component of the longitudinal deviatoric stress is $\tau_{\mathrm{xx}}^{\prime}=\tau_{\mathrm{xx}}-\frac{1}{3}\left(\tau_{\mathrm{xx}}+\tau_{\mathrm{yy}}+\tau_{\mathrm{zz}}\right)=0$, and analogue for the $y$ and the $z$ component. For hydrostatic equilibrium we have thus

$\tau_{\mathrm{xx}}=\tau_{\mathrm{yy}}=\tau_{\mathrm{zz}}$

${ }_{10}^{\prime} \tau_{\mathrm{xx}}^{\prime}=\tau_{\mathrm{yy}}^{\prime}=\tau_{\mathrm{zz}}^{\prime}=0$

A3 Incompressibility

Incompressibility is imposed by

$\tau_{\mathrm{xx}}^{\prime}+\tau_{\mathrm{yy}}^{\prime}+\tau_{\mathrm{zz}}^{\prime}=0$

2, 81-158, 2009

A numerical ice sheet

- ice shelf 3D

velocity field solution

T. J. Reerink et al.

Hydrostatic equilibrium is a stronger restriction than incompressibility, because incompressibility requires $\tau_{\mathrm{ii}}^{\prime}=0$ which not necessarily implies Eq. (A4). In ice dynamics incompressibility is assumed everywhere (at the sheet, the shelf and in the transition zone), while hydrostatic equilibrium is only valid at most parts of the sheet.

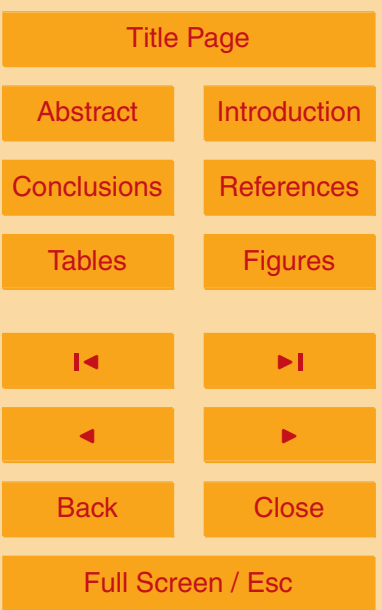

Printer-friendly Version

Interactive Discussion 
A4 The derivation of the deviatoric stress equations

The horizontal normal deviatoric stresses, being in general non-zero if there is no hydrostatic equilibrium, are with use of Eq. (A2)

$\tau_{\mathrm{xx}}^{\prime}=\frac{2}{3} \tau_{\mathrm{xx}}-\frac{1}{3} \tau_{\mathrm{yy}}-\frac{1}{3} \tau_{\mathrm{zz}}$

${ }_{5} \quad \tau_{\mathrm{yy}}^{\prime}=\frac{2}{3} \tau_{\mathrm{yy}}-\frac{1}{3} \tau_{\mathrm{xx}}-\frac{1}{3} \tau_{\mathrm{zz}}$

Taking six times Eq. (A6) and adding or subtracting three times Eq. (A7) leads respectively to

$$
\begin{aligned}
& \tau_{\mathrm{xx}}=\tau_{\mathrm{zz}}+2 \tau_{\mathrm{xx}}^{\prime}+\tau_{\mathrm{yy}}^{\prime} \\
& \tau_{\mathrm{yy}}=\tau_{\mathrm{zz}}+2 \tau_{\mathrm{yy}}^{\prime}+\tau_{\mathrm{xx}}^{\prime}
\end{aligned}
$$

10 Substitution of Eqs. (A8-A9) respectively into Eqs. (3.8-3.9) gives

$\partial_{z} \tau_{\mathrm{xz}}=-\partial_{x}\left(\tau_{\mathrm{zz}}+2 \tau_{\mathrm{xx}}^{\prime}+\tau_{\mathrm{yy}}^{\prime}\right)-\partial_{y} \tau_{\mathrm{xy}}$

$\partial_{z} \tau_{\mathrm{yz}}=-\partial_{y}\left(\tau_{\mathrm{zz}}+2 \tau_{\mathrm{yy}}^{\prime}+\tau_{\mathrm{xx}}^{\prime}\right)-\partial_{x} \tau_{\mathrm{yx}}$

Substituting for $\tau_{\mathrm{zz}}$ Eq. (3.14) into Eqs. (A10-A11) and using $\tau_{\mathrm{xy}}=\tau_{\mathrm{yx}}$ because the stress tensor $\tau_{\mathrm{ij}}$ is symmetric, we get:

$$
\begin{aligned}
& \partial_{z} \tau_{\mathrm{xz}}=\rho_{\mathrm{ice}} g \partial_{x} H_{s}-2 \partial_{x} \tau_{\mathrm{xx}}^{\prime}-\partial_{x} \tau_{\mathrm{yy}}^{\prime}-\partial_{y} \tau_{\mathrm{xy}} \\
& \partial_{z} \tau_{\mathrm{yz}}=\rho_{\mathrm{ice}} g \partial_{y} H_{s}-2 \partial_{y} \tau_{\mathrm{yy}}^{\prime}-\partial_{y} \tau_{\mathrm{xx}}^{\prime}-\partial_{x} \tau_{\mathrm{xy}}
\end{aligned}
$$

Because each non-diagonal component of the stress tensor equals the corresponding component of the deviatoric stress tensor, we can express Eqs. (A12-A13) in deviatoric

\section{GMDD}

2, 81-158, 2009

A numerical ice sheet - ice shelf 3D velocity field solution

T. J. Reerink et al.

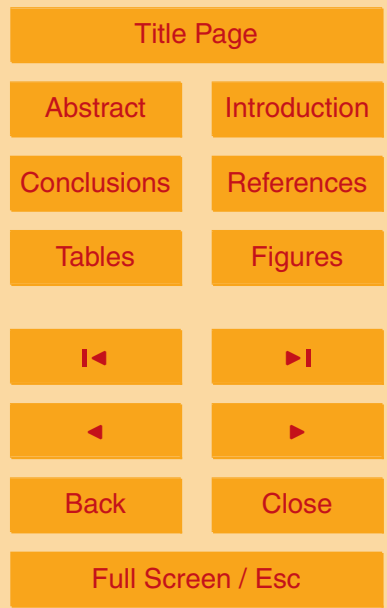

Printer-friendly Version

Interactive Discussion 
components only

$$
\begin{aligned}
\partial_{z} \tau_{\mathrm{xz}}^{\prime} & =\rho_{\mathrm{ice}} g \partial_{x} H_{s}-2 \partial_{x} \tau_{\mathrm{xx}}^{\prime}-\partial_{x} \tau_{\mathrm{yy}}^{\prime}-\partial_{y} \tau_{\mathrm{xy}}^{\prime} \\
\partial_{z} \tau_{\mathrm{yz}}^{\prime} & =\rho_{\mathrm{ice}} g \partial_{y} H_{s}-2 \partial_{y} \tau_{\mathrm{yy}}^{\prime}-\partial_{y} \tau_{\mathrm{xx}}^{\prime}-\partial_{x} \tau_{\mathrm{xy}}^{\prime} \\
\tau_{\mathrm{zz}}^{\prime} & =-\tau_{\mathrm{xx}}^{\prime}-\tau_{\mathrm{yy}}^{\prime}
\end{aligned}
$$

5 As a third equation the incompressibility condition A5 has been added.

\section{Appendix B}

\section{The transformed derivatives accompanying a coordinate transformation}

\section{B1 The transformed gradients}

10 Having a coordinate system $A$ with coordinates $\boldsymbol{x}$, where $\boldsymbol{x}$ is a vector containing all the coordinates of $A$, and having a derivative operator working on a function $f$, which takes the derivative to one of the coordinates of $x$, we want to transform this derivative operator to another coordinate system $B$ with coordinates $\boldsymbol{x}^{\prime}$. The general transformation rule or chain rule, for a transformation from system $A$ to $B$ of the operator which takes the $x_{\mathrm{i}}$-derivative of a function $f$, is then

$\partial_{x_{\mathrm{i}}} f=\left(\partial_{x_{\mathrm{j}}^{\prime}} f\right) \partial_{x_{\mathrm{i}}} x_{\mathrm{j}}^{\prime}$

with the summation convention, $\boldsymbol{x}=\left\{x_{1}, x_{2}, \ldots, x_{n}\right\}$ and $\boldsymbol{x}^{\prime}=\left\{x_{1}^{\prime}, x_{2}^{\prime}, \ldots, x_{n}^{\prime}\right\}$. In principle $f$ might be a function of the old $x$ or the new $x^{\prime}$ coordinates. The transformation rule only takes the correction for the derivative operator into account and not the eventually

transformation of $f$. E.g. in our cases $f$ will usually be expressed in the old coordinates $\boldsymbol{x}$ at the left hand side, while at the right hand side $f$ will be expressed in the new

GMDD

2, 81-158, 2009

A numerical ice sheet - ice shelf 3D velocity field solution

T. J. Reerink et al.

Title Page

Abstract

Introduction

Conclusions

References

Tables

Figures

14

$>1$

4

Back

Close 
coordinates $x^{\prime}$. In our specific case we have $x=\{t, x, y, z\}$ and $x^{\prime}=\left\{t^{\prime}, x^{\prime}, y^{\prime}, \zeta\right\}$, which leads with Eq. (B1) to

$\partial_{x_{\mathrm{i}}} f(t, x, y, z)=\left(\partial_{x_{\mathrm{j}}^{\prime}} f\left(t^{\prime}, x^{\prime}, y^{\prime}, \zeta\right)\right) \partial_{x_{\mathrm{i}}} x_{\mathrm{j}}^{\prime}$

where in general $t^{\prime}, x^{\prime}, y^{\prime}, \zeta$ don't have to be independent variables. In our case $\zeta$ is a 5 (non-equidistant) scaled coordinate. $\zeta$ is scaled for each grid point and for each time step. This means $\zeta$ varies with $x, y$, and $t$, causing 'correction terms' for the $x, y$, and $t$ derivatives of $f$ when transformed to system $B$. For example, if we take $x_{\mathrm{i}}=t$, we get

$\partial_{t} f=\left(\partial_{t^{\prime}} f\right) \partial_{t} t^{\prime}+\left(\partial_{x^{\prime}} f\right) \partial_{t} x^{\prime}+\left(\partial_{y^{\prime}} f\right) \partial_{t^{\prime}} y^{\prime}+\left(\partial_{\zeta} f\right) \partial_{t} \zeta$

In our case the transformation actually concerns only the vertical coordinate $z$, so $t=t$, $10 x^{\prime}=x$, and $y^{\prime}=y$, and thus $\partial_{t} t^{\prime}=1, \partial_{t} x^{\prime}=\partial_{t} y^{\prime}=0$ and $\partial_{t} \zeta \neq 0$, etc. Using these values results in

$\partial_{t} f=\partial_{t^{\prime}} f+\left(\partial_{\zeta} f\right) \partial_{t} \zeta=\partial_{t^{\prime}} f+\zeta_{t} \partial_{\zeta} f$

Analogue, we obtain for the remaining transformed gradients of $f$ :

$\partial_{x} f=\partial_{x^{\prime}} f+\left(\partial_{\zeta} f\right) \partial_{x} \zeta=\partial_{x^{\prime}} f+\zeta_{x} \partial_{\zeta} f$

$\partial_{y} f=\partial_{y^{\prime}} f+\left(\partial_{\zeta} f\right) \partial_{y} \zeta=\partial_{y^{\prime}} f+\zeta_{y} \partial_{\zeta} f$

$\partial_{z} f=$

$\left(\partial_{\zeta} f\right) \partial_{z} \zeta=$

$\zeta_{z} \partial_{\zeta} f$

Where the $\zeta$ 's with a lower index are a short notation for the derivative of $\zeta$ to the variable indicated by the index.

In our case the vertical coordinate $z$ is transformed to the (non-equidistant) scaled
GMDD

2, 81-158, 2009

A numerical ice sheet

- ice shelf 3D

velocity field solution

T. J. Reerink et al.

Title Page

Abstract

Introduction

Conclusions

Tables

References

Figures

14

$\rightarrow$

4

Back

Close

Full Screen / Esc

Printer-friendly Version

Interactive Discussion

$\zeta(t, x, y, z)=\frac{H_{s}(t, x, y)-z}{H(t, x, y)}$ 
where $\zeta=0$ at the ice surface, and $\zeta=1$ at the ice-bedrock interface (see Fig. 2). With the product rule (and with $\partial_{t} z=\partial_{x} z=\partial_{y} z=0$ and $\partial_{z} H_{s}=\partial_{z} H=0$, because $H_{s}$ and $H$ are two-dimensional fields which are independent of the vertical coordinate) the derivatives of $\zeta$ become

${ }_{5} \zeta_{\mathrm{t}} \equiv \partial_{t} \zeta=\frac{1}{H}\left(\partial_{t} H_{s}-\zeta \partial_{t} H\right)$

$\zeta_{x} \equiv \partial_{x} \zeta=\frac{1}{H}\left(\partial_{x} H_{s}-\zeta \partial_{x} H\right)$

$\zeta_{y} \equiv \partial_{y} \zeta=\frac{1}{H}\left(\partial_{y} H_{s}-\zeta \partial_{y} H\right)$

$\zeta_{z} \equiv \partial_{z} \zeta=-\frac{1}{H}$

B2 The transformed second derivatives

10 The "simple chain rule" is used to transform a derivative of a function to an old coordinate towards a derivative of a function to a new coordinate. In case that more coordinates are involved the "chain rule for several variables" as in Eq. (B1) should be used. In case the coordinate transformation concerns a single coordinate system, higher derivative operators can be transformed with use of Faà di Bruno's formula which is the "generalized chain rule for higher derivatives". Here, we derive a general chain rule for the specific case of second derivatives but for the case of several coordinates (or variables).

To derive the transformation rules for second derivatives working on a function $f$ we consider the chain rule for the gradient Eq. (B1)

$$
\partial_{x_{\mathrm{j}}} f=\left(\partial_{x_{\mathrm{n}}^{\prime}} f\right)\left(\partial_{x_{\mathrm{j}}} x_{\mathrm{n}}^{\prime}\right)
$$

with the summation convention for the primed variables. Now we take the $x_{\mathrm{i}}$-derivative

\section{GMDD}

2, 81-158, 2009

A numerical ice sheet - ice shelf 3D velocity field solution

T. J. Reerink et al.

Title Page

\section{Abstract}

Introduction

Conclusions

Tables

References Figures

14 I

4

Back

Close

Full Screen / Esc

Printer-friendly Version

Interactive Discussion 
of this equation:

$\partial_{x_{\mathrm{i}}}\left(\partial_{x_{\mathrm{j}}} f\right)=\partial_{x_{\mathrm{i}}}\left[\left(\partial_{x_{\mathrm{n}}^{\prime}} f\right)\left(\partial_{x_{\mathrm{j}}} x_{\mathrm{n}}^{\prime}\right)\right]$

Using the product rule, we get

$\partial_{x_{\mathrm{i}} x_{\mathrm{j}}}^{2} f=\left(\partial_{x_{\mathrm{i}}}\left[\partial_{x_{\mathrm{n}}^{\prime}} f\right]\right)\left(\partial_{x_{\mathrm{j}}} x_{\mathrm{n}}^{\prime}\right)+\left(\partial_{x_{\mathrm{n}}^{\prime}} f\right)\left(\partial_{x_{\mathrm{i}} x_{\mathrm{j}}}^{2} x_{\mathrm{n}}^{\prime}\right)$

2, 81-158, 2009

5 Because in the first term at the right hand side $\partial_{x_{\mathrm{i}}}\left[\partial_{x_{n}^{\prime}} f\right]$ is the old coordinate $x_{\mathrm{i}^{-}}$ derivative, we need the chain rule again to express all derivatives of $f$ in the previous equation as derivatives to the new (the primed) coordinates. This is done in the next step:

$\partial_{x_{\mathrm{i}} x_{\mathrm{j}}}^{2} f=\left(\partial_{x_{\mathrm{m}}^{\prime} x_{\mathrm{n}}^{\prime}}^{2} f\right)\left(\partial_{x_{\mathrm{i}}} x_{\mathrm{m}}^{\prime}\right)\left(\partial_{x_{\mathrm{j}}} x_{\mathrm{n}}^{\prime}\right)+\left(\partial_{x_{\mathrm{n}}^{\prime}}^{\prime}\right)\left(\partial_{x_{\mathrm{i}} x_{\mathrm{j}}}^{2} x_{\mathrm{n}}^{\prime}\right)$

10 with the summation convention for the primed variables $x_{m}^{\prime}$ and $x_{n}^{\prime}$. Usually, but not necessary, $f$ at the left hand side is expressed in the old coordinates and $f$ at the right hand side in the new coordinates.

Substituting $x_{\mathrm{i}}=x_{\mathrm{j}}=x$ in Eq. (B13), we get

$\partial_{x x}^{2} f=\left(\partial_{x_{\mathrm{m}}^{\prime} x_{\mathrm{n}}^{\prime}}^{2} f\right)\left(\partial_{x} x_{\mathrm{m}}^{\prime}\right)\left(\partial_{x} x_{\mathrm{n}}^{\prime}\right)+\left(\partial_{x_{\mathrm{n}}^{\prime}} f\right)\left(\partial_{x x}^{2} x_{\mathrm{n}}^{\prime}\right)$

15 Do we substitute $x_{\mathrm{i}}=x$ and $x_{\mathrm{j}}=y$ in Eq. (B13), we get

$\partial_{x y}^{2} f=\left(\partial_{x_{\mathrm{m}}^{\prime} x_{\mathrm{n}}^{\prime}}^{2} f\right)\left(\partial_{x} x_{\mathrm{m}}^{\prime}\right)\left(\partial_{y} x_{\mathrm{n}}^{\prime}\right)+\left(\partial_{x_{\mathrm{n}}^{\prime}} f\right)\left(\partial_{x y}^{2} x_{\mathrm{n}}^{\prime}\right)$

A numerical ice sheet

- ice shelf 3D

velocity field solution

T. J. Reerink et al.

Finally, substitution of $x_{\mathrm{i}}=x$ and $x_{\mathrm{j}}=z$ in Eq. (B13) gives

$\partial_{x z}^{2} f=\left(\partial_{x_{\mathrm{m}}^{\prime} x_{\mathrm{n}}^{\prime}}^{2} f\right)\left(\partial_{x} x_{\mathrm{m}}^{\prime}\right)\left(\partial_{z} x_{\mathrm{n}}^{\prime}\right)+\left(\partial_{x_{\mathrm{n}}^{\prime}} f\right)\left(\partial_{x z}^{2} x_{\mathrm{n}}^{\prime}\right)$

Printer-friendly Version

Interactive Discussion 
In our case, in fact only the vertical coordinate is transformed. The other three coordinates remain equal. In that case, Eq. (B14) reduces to

$\partial_{x x}^{2} f=\partial_{x^{\prime} x^{\prime}}^{2} f+2\left(\partial_{x} \zeta\right)\left(\partial_{x^{\prime} \zeta}^{2} f\right)+\left(\partial_{x} \zeta\right)^{2}\left(\partial_{\zeta \zeta}^{2} f\right)+\left(\partial_{x x}^{2} \zeta\right)\left(\partial_{\zeta} f\right)$

GMDD

while Eq. (B15) becomes

$\partial_{x y}^{2} f=\partial_{x^{\prime} y^{\prime}}^{2} f+\left(\partial_{y} \zeta\right)\left(\partial_{x^{\prime} \zeta}^{2} f\right)+\left(\partial_{x} \zeta\right)\left(\partial_{y^{\prime} \zeta}^{2} f\right)+\left(\partial_{x} \zeta\right)\left(\partial_{y} \zeta\right)\left(\partial_{\zeta \zeta}^{2} f\right)+\left(\partial_{x y}^{2} \zeta\right)\left(\partial_{\zeta} f\right)$

and Eq. (B16) simplifies to

$\partial_{x z}^{2} f=\left(\partial_{z} \zeta\right)\left(\partial_{x^{\prime} \zeta}^{2} f\right)+\left(\partial_{x} \zeta\right)\left(\partial_{z} \zeta\right)\left(\partial_{\zeta \zeta}^{2} f\right)+\left(\partial_{x z}^{2} \zeta\right)\left(\partial_{\zeta} f\right)$

Finally, these Eqs. (B17-B19) and their equivalents can then be written as

$\partial_{x x}^{2} f=\partial_{x^{\prime} x^{\prime}}^{2} f+2 \zeta_{x} \partial_{x^{\prime} \zeta}^{2} f+\zeta_{x}^{2} \partial_{\zeta \zeta}^{2} f+\zeta_{x x} \partial_{\zeta} f$

$\partial_{x y}^{2} f=\partial_{x^{\prime} y^{\prime}}^{2} f+\zeta_{y} \partial_{x^{\prime} \zeta}^{2} f+\zeta_{x} \partial_{y^{\prime} \zeta}^{2} f+\zeta_{x} \zeta_{y} \partial_{\zeta \zeta}^{2} f+\zeta_{x y} \partial_{\zeta} f$

$\partial_{x z}^{2} f=\zeta_{z} \partial_{x^{\prime} \zeta}^{2} f+\zeta_{x} \zeta_{z} \partial_{\zeta \zeta}^{2} f+\zeta_{x z} \partial_{\zeta} f$

$\partial_{y y}^{2} f=\partial_{y^{\prime} y^{\prime}}^{2} f+2 \zeta_{\mathrm{y}} \partial_{y^{\prime} \zeta}^{2} f+\zeta_{\mathrm{y}}^{2} \partial_{\zeta \zeta}^{2} f+\zeta_{\mathrm{yy}} \partial_{\zeta} f$

$\partial_{y z}^{2} f=\zeta_{z} \partial_{y^{\prime} \zeta}^{2} f+\zeta_{y} \zeta_{z} \partial_{\zeta \zeta}^{2} f+\zeta_{y z} \partial_{\zeta} f$

$\partial_{z z}^{2} f=\zeta_{z}^{2} \partial_{\zeta \zeta}^{2} f$

15 The various coefficients are defined at the bottom of Table 3.

2, 81-158, 2009

A numerical ice sheet - ice shelf 3D velocity field solution

T. J. Reerink et al.

Title Page

Abstract

Introduction

Conclusions

References

Tables

Figures

14

14

4

Back

Close

Full Screen / Esc

Printer-friendly Version

Interactive Discussion 


\section{Appendix C}

\section{Discretization rules for derivatives}

\section{GMDD}

2, 81-158, 2009

With a Taylor expansion a value of a function $f(x)$ in a point $x$ can be approximated 5 with a value $f\left(x_{0}\right)$, for a value $x_{0}$ near $x$, up to $n$-th order accuracy

$f(x)=\sum_{m=0}^{n}\left(\frac{f^{m}(x)}{m !}\right)_{x=x_{0}}\left(x-x_{0}\right)^{m}$

To obtain the discretization for a gradient or any $n$-th derivative of a function $f$, we consider the Taylor approximation within a point being a few grid steps ahead of the point $\left(x_{0}\right)$, around which the approximation will occur. Numerical calculations are performed on a grid. The grid sizes will determine the smallest steps. In general this grid size might be non-equidistant. We take as a non-equidistant coordinate $\zeta$, labeled by $\mathrm{k}$ for each grid cell. The grid size will be $\mathrm{d} \zeta$. Both, $\zeta$ and $\mathrm{k}$ have the same direction. Let $\zeta_{\mathrm{k}}$ correspond with $\zeta_{0}$ in the Taylor expansion. We will calculate the approximation of $f\left(\zeta_{\mathrm{k}}+p \mathrm{~d} \zeta\right)$ around $\zeta_{\mathrm{k}}$. The taylor expansion for a point $p \mathrm{~d} \zeta$ ahead of $\zeta_{\mathrm{k}}$ becomes

$f\left(\zeta_{\mathrm{k}}+p \mathrm{~d} \zeta\right)=\sum_{m=0}^{n}\left(\frac{f^{m}\left(\zeta_{\mathrm{k}}\right)}{m !}\right)\left(\left(\zeta_{\mathrm{k}}+p \mathrm{~d} \zeta\right)-\zeta_{\mathrm{k}}\right)^{m}$

$f_{\mathrm{k}+\mathrm{p}}=\sum_{m=0}^{n}\left(\partial_{\zeta^{m}}^{m} f\right)_{\mathrm{k}}\left(\frac{\zeta_{\mathrm{k}+\mathrm{p}}-\zeta_{\mathrm{k}}}{m !}\right)^{m}$

with $\zeta_{\mathrm{k}+\mathrm{p}} \equiv \zeta_{\mathrm{k}}+p \mathrm{~d} \zeta$. With this rule the central, one-sided, or mixed (e.g. upsteam) discretization of an $\mathrm{n}$-th derivative of any order can be constructed. In case of an equidistant grid we have $\zeta_{\mathrm{k}+\mathrm{p}}-\zeta_{\mathrm{k}}=p \Delta x$. Using $x$ and $\mathrm{i}$ instead of $\zeta$ and $\mathrm{k}$, and noting

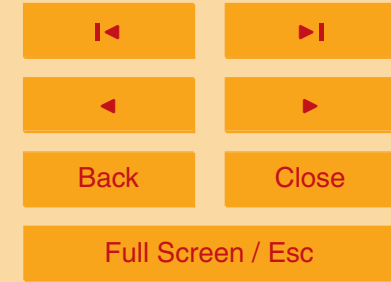

A numerical ice sheet - ice shelf 3D velocity field solution

T. J. Reerink et al.

\section{Title Page}

Abstract Introduction

Conclusions References

Tables Figures

Printer-friendly Version

Interactive Discussion 
that $x$ and i point in the same direction, Eq. (C3) becomes in the equidistant case

$f_{\mathrm{i}+\mathrm{p}}=\sum_{m=0}^{n} \frac{p^{m}}{m !}\left(\partial_{x^{m}}^{m} f\right)_{\mathrm{i}}(\Delta x)^{m}$

GMDD

Taking Eq. (C4) with $n=2$ for $p=-1, p=1, p=-2$ and $p=2$ we get respectively

$f_{\mathrm{i}-1}=f_{\mathrm{i}}-\left(\partial_{x} f\right)_{\mathrm{i}} \Delta x+\frac{1}{2}\left(\partial_{x x}^{2} f\right)_{\mathrm{i}}(\Delta x)^{2}+O\left((\Delta x)^{3}\right)$

$5 \quad f_{\mathrm{i}+1}=f_{\mathrm{i}}+\left(\partial_{x} f\right)_{\mathrm{i}} \Delta x+\frac{1}{2}\left(\partial_{x x}^{2} f\right)_{\mathrm{i}}(\Delta x)^{2}+O\left((\Delta x)^{3}\right)$

$f_{\mathrm{i}-2}=f_{\mathrm{i}}-2\left(\partial_{x} f\right)_{\mathrm{i}} \Delta x+2\left(\partial_{x x}^{2} f\right)_{\mathrm{i}}(\Delta x)^{2}+O\left((\Delta x)^{3}\right)$

$f_{\mathrm{i}+2}=f_{\mathrm{i}}+2\left(\partial_{x} f\right)_{\mathrm{i}} \Delta x+2\left(\partial_{x x}^{2} f\right)_{\mathrm{i}}(\Delta x)^{2}+O\left((\Delta x)^{3}\right)$

To obtain a central second order discretization for the gradient and the second derivative of $f$, Eqs. (C5-C6) have to be added and subtracted respectively

$10 \quad\left(\partial_{x} f\right)_{\mathrm{i}}=\frac{f_{\mathrm{i}+1}-f_{\mathrm{i}-1}}{2 \Delta x}$

$\left(\partial_{x x}^{2} f\right)_{\mathrm{i}}=\frac{f_{\mathrm{i}-1}+f_{\mathrm{i}+1}-2 f_{\mathrm{i}}}{(\Delta x)^{2}}$

Taking Eq. (C4) with $n=1$ for $p=-1$

$f_{\mathrm{i}-1}=f_{\mathrm{i}}-\left(\partial_{x} f\right)_{\mathrm{i}} \Delta x+O\left((\Delta x)^{2}\right)$

immediately produces the first order gradient

${ }_{15}\left(\partial_{X} f\right)_{\mathrm{i}}=\frac{f_{\mathrm{i}}-f_{\mathrm{i}-1}}{\Delta x}$

For the more general eventually non-equidistant case, we take Eq. (C3) with $n=2$ for

A numerical ice sheet

- ice shelf 3D

velocity field solution

T. J. Reerink et al.

Title Page

Abstract

Introduction

Conclusions

Tables

Figures

14

$>$ I

4

Back

Close

Full Screen / Esc

Printer-friendly Version

Interactive Discussion 


$$
\begin{aligned}
& p=-1, p=1, p=-2 \text { and } p=+2 \\
& f_{\mathrm{k}-1}=f_{\mathrm{k}}+\left(\partial_{\zeta} f\right)_{\mathrm{k}}\left(\zeta_{\mathrm{k}-1}-\zeta_{\mathrm{k}}\right)+\frac{1}{2}\left(\partial_{\zeta \zeta}^{2} f\right)_{\mathrm{k}}\left(\zeta_{\mathrm{k}-1}-\zeta_{\mathrm{k}}\right)^{2}+O\left((\Delta x)^{3}\right) \\
& =f_{\mathrm{k}}-a\left(\partial_{\zeta} f\right)_{\mathrm{k}}+\frac{1}{2} a^{2}\left(\partial_{\zeta \zeta}^{2} f\right)_{\mathrm{k}}+O\left((\Delta x)^{3}\right) \\
& f_{\mathrm{k}+1}=f_{\mathrm{k}}+\left(\partial_{\zeta} f\right)_{\mathrm{k}}\left(\zeta_{\mathrm{k}+1}-\zeta_{\mathrm{k}}\right)+\frac{1}{2}\left(\partial_{\zeta \zeta}^{2} f\right)_{\mathrm{k}}\left(\zeta_{\mathrm{k}+1}-\zeta_{\mathrm{k}}\right)^{2}+O\left((\Delta x)^{3}\right) \\
& =f_{\mathrm{k}}+b\left(\partial_{\zeta} f\right)_{\mathrm{k}}+\frac{1}{2} b^{2}\left(\partial_{\zeta \zeta}^{2} f\right)_{\mathrm{k}}+O\left((\Delta x)^{3}\right) \\
& f_{\mathrm{k}-2}=f_{\mathrm{k}}+\left(\partial_{\zeta} f\right)_{\mathrm{k}}\left(\zeta_{\mathrm{k}-2}-\zeta_{\mathrm{k}}\right)+\frac{1}{2}\left(\partial_{\zeta \zeta}^{2} f\right)_{\mathrm{k}}\left(\zeta_{\mathrm{k}-2}-\zeta_{\mathrm{k}}\right)^{2}+O\left((\Delta x)^{3}\right) \\
& =f_{\mathrm{k}}-c\left(\partial_{\zeta} f\right)_{\mathrm{k}}+\frac{1}{2} c^{2}\left(\partial_{\zeta \zeta}^{2} f\right)_{\mathrm{k}}+O\left((\Delta x)^{3}\right) \\
& f_{\mathrm{k}+2}=f_{\mathrm{k}}+\left(\partial_{\zeta} f\right)_{\mathrm{k}}\left(\zeta_{\mathrm{k}+2}-\zeta_{\mathrm{k}}\right)+\frac{1}{2}\left(\partial_{\zeta \zeta}^{2} f\right)_{\mathrm{k}}\left(\zeta_{\mathrm{k}+2}-\zeta_{\mathrm{k}}\right)^{2}+O\left((\Delta x)^{3}\right) \\
& =f_{\mathrm{k}}+d\left(\partial_{\zeta} f\right)_{\mathrm{k}}+\frac{1}{2} d^{2}\left(\partial_{\zeta \zeta}^{2} f\right)_{\mathrm{k}}+O\left((\Delta x)^{3}\right)
\end{aligned}
$$

10 where

$$
\begin{aligned}
a & =a_{k} \equiv \zeta_{\mathrm{k}}-\zeta_{\mathrm{k}-1} \\
b & =b_{k} \equiv \zeta_{\mathrm{k}+1}-\zeta_{\mathrm{k}} \\
c & =c_{k} \equiv \zeta_{\mathrm{k}}-\zeta_{\mathrm{k}-2} \\
d & =d_{k} \equiv \zeta_{\mathrm{k}+2}-\zeta_{\mathrm{k}}
\end{aligned}
$$

A numerical ice sheet - ice shelf 3D

\section{velocity field solution}

T. J. Reerink et al.

Title Page

Abstract

Introduction

Conclusions

Tables

References

Figures

14

$\rightarrow$

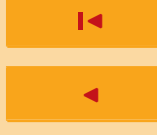

Back

Close

Full Screen / Esc

Printer-friendly Version

Interactive Discussion

$\left(\partial_{\zeta} f\right)_{\mathrm{k}}=\frac{a^{2} f_{\mathrm{k}+1}-b^{2} f_{\mathrm{k}-1}-\left(a^{2}-b^{2}\right) f_{\mathrm{k}}}{a b(a+b)}$ 
To obtain a second order central discretization of the second derivative on an eventually non-equidistant grid, we take a times Eq. (C14) and add $b$ times Eq. (C13)

$$
\left(\partial_{\zeta \zeta}^{2} f\right)_{\mathrm{k}}=\frac{a f_{\mathrm{k}+1}+b f_{\mathrm{k}-1}-(a+b) f_{\mathrm{k}}}{\frac{1}{2} a b(a+b)}
$$

The rest term of Eq. (C22) is

5 rest term $=-\frac{1}{3} \frac{b^{2}-a^{2}}{a+b}\left(\partial_{\zeta^{3}}^{3} f\right)_{\mathrm{k}}-\frac{1}{12} \frac{a^{3}+b^{3}}{a+b}\left(\partial_{\zeta^{4}}^{4} f\right)_{\mathrm{k}}$

from which is seen that the first term might be second order if the grid is near to equidistant, but could be first order. This implies that we have to be careful with taking Eq. (C22) as a second order accuracy discretization.

Taking Eq. (C3) with $n=1$ for $p=-1$

$10 f_{\mathrm{k}-1}=f_{\mathrm{k}}+\left(\partial_{\zeta} f\right)_{\mathrm{k}} \mathrm{a}+O\left((\Delta x)^{2}\right)$

we directly get the first order gradient on an eventually non-equidistant grid

$\left(\partial_{\zeta} f\right)_{\mathrm{k}}=\frac{f_{\mathrm{k}}-f_{\mathrm{k}-1}}{a}$

In case of an equidistant grid, $a=b=\Delta x$ Eqs. (C21-C22) reduce to Eqs. (C9-C10) respectively and Eq. (C25) reduces to Eq. (C12).

C1 Alternative notation for central discretizations

In complicated cases it might be handier to use a simple notation which also makes a efficient coding possible. The central non-equidistant discretizations of the first and second derivative Eqs. (C21-C22) can be written as

$$
\left(\partial_{\zeta} f\right)_{\mathrm{k}}=a_{\zeta} f_{\mathrm{k}-1}+b_{\zeta} f_{\mathrm{k}}+c_{\zeta} f_{\mathrm{k}+1}
$$

\section{GMDD}

2, 81-158, 2009

A numerical ice sheet - ice shelf 3D velocity field solution

T. J. Reerink et al.

Title Page

\section{Abstract}

Introduction

Conclusions

Tables

References

Figures

14

-I

4

Back

Close

Full Screen / Esc

Printer-friendly Version

Interactive Discussion 
where the coefficients are defined in Table 4.

Equation (C26) becomes shorter for the equidistant case (because $a=b$ and thus $\left.b_{x}=0\right)$

$$
\left(\partial_{x} f\right)_{\mathrm{i}}=a_{x} f_{\mathrm{i}-1}+c_{x} f_{\mathrm{i}+1}
$$

5 where the coefficients are defined in Table 4.

\section{C2 Mixed second derivatives}

Although not directly derived from a combination of Taylor series, like we did before with Eq. (C3), the mixed second derivatives are obtained by applying a sequence of Eq. (C26) and Eq. (C28) for a three-dimensional field variable. The lower indices i and

$10 \mathrm{j}$ represent the horizontal equidistant grid directions $x$ and $y$, while the upper index $\mathrm{k}$ represents the vertical non equidistant direction $\zeta$.

$$
\begin{aligned}
& \left(\partial_{x y}^{2} f\right)_{\mathrm{k}}=a_{x} a_{y} f_{\mathrm{i}-1, \mathrm{j}-1}^{\mathrm{k}}+a_{x} c_{y} f_{\mathrm{i}-1, \mathrm{j}+1}^{\mathrm{k}}+c_{x} a_{y} f_{\mathrm{i}+1, \mathrm{j}-1}^{\mathrm{k}}+c_{x} c_{y} f_{\mathrm{i}+1, \mathrm{j}+1}^{\mathrm{k}} \\
& \left(\partial_{x \zeta}^{2} f\right)_{\mathrm{k}}=a_{\zeta} a_{x} f_{\mathrm{i}-1, \mathrm{j}}^{\mathrm{k}-1}+a_{\zeta} c_{x} f_{\mathrm{i}+1, \mathrm{j}}^{\mathrm{k}-1}+b_{\zeta} a_{x} f_{\mathrm{i}-1, \mathrm{j}}^{\mathrm{k}}+b_{\zeta} c_{x} f_{\mathrm{i}+1, \mathrm{j}}^{\mathrm{k}}+c_{\zeta} a_{x} f_{\mathrm{i}-1, \mathrm{j}}^{\mathrm{k}+1}+c_{\zeta} c_{x} f_{\mathrm{i}+1, \mathrm{j}}^{\mathrm{k}+1} \\
& \left(\partial_{y \zeta}^{2} f\right)_{\mathrm{k}}=a_{\zeta} a_{y} f_{\mathrm{i}, \mathrm{j}-1}^{\mathrm{k}-1}+a_{\zeta} c_{y} f_{\mathrm{i}, \mathrm{j}+1}^{\mathrm{k}-1}+b_{\zeta} a_{y} f_{\mathrm{i}, \mathrm{j}-1}^{\mathrm{k}}+b_{\zeta} c_{y} f_{\mathrm{i}, \mathrm{j}+1}^{\mathrm{k}}+c_{\zeta} a_{y} f_{\mathrm{i}, \mathrm{j}-1}^{\mathrm{k}+1}+c_{\zeta} c_{y} f_{\mathrm{i}, \mathrm{j}+1}^{\mathrm{k}+1}
\end{aligned}
$$

15 where the coefficients are defined in Table 4.

\section{GMDD}

2, 81-158, 2009

A numerical ice sheet - ice shelf 3D velocity field solution

T. J. Reerink et al.

\section{Title Page}

\section{Abstract}

Introduction

Conclusions

Tables

References

Figures

14

$\rightarrow$

\section{4}

Back

\section{Full Screen / Esc}

Printer-friendly Version 


\section{Appendix D}

\section{Transformation and discretization}

2, 81-158, 2009

Starting from Eqs. (4.1-4.3):

$5 \frac{1}{2} f_{4} \partial_{z}\left(C^{\mathrm{uvw}}\left(\partial_{z} u+f_{0} \partial_{x} w\right)\right)+2 f_{5} \partial_{x}\left(C^{\mathrm{uvw}} \partial_{x} u\right)+f_{5} \partial_{x}\left(C^{\mathrm{uvw}} \partial_{y} v\right)$

$$
+\frac{1}{2} f_{6} \partial_{y}\left(C^{\mathrm{uvw}}\left(\partial_{y} u+\partial_{x} v\right)\right)=\rho_{\text {ice }} g \partial_{x} H_{s}
$$

$\frac{1}{2} f_{4} \partial_{z}\left(C^{\mathrm{uvw}}\left(\partial_{z} v+f_{0} \partial_{y} w\right)\right)+2 f_{5} \partial_{y}\left(C^{\mathrm{uvw}} \partial_{y} v\right)+f_{5} \partial_{y}\left(C^{\mathrm{uvw}} \partial_{x} u\right)$

$$
\begin{aligned}
+\frac{1}{2} f_{6} \partial_{x}\left(C^{\mathrm{uvw}}\left(\partial_{y} u+\partial_{x} v\right)\right) & =\rho_{\text {ice }} g \partial_{y} H_{s} \\
\partial_{x} u+\partial_{y} v+\partial_{z} w & =0
\end{aligned}
$$

10 We work out the parenthesis:

$$
\begin{aligned}
f_{4} \partial_{z}\left(C^{\mathrm{uvw}} \partial_{z} u\right)+f_{4} f_{0} \partial_{z}\left(C^{\mathrm{uvw}} \partial_{x} w\right)+4 f_{5} \partial_{x}\left(C^{\mathrm{uvw}} \partial_{x} u\right) & \\
+2 f_{5} \partial_{x}\left(C^{\mathrm{uvw}} \partial_{y} v\right)+f_{6} \partial_{y}\left(C^{\mathrm{uvw}}\left(\partial_{y} u+\partial_{x} v\right)\right) & =2 \rho_{\mathrm{ice}} g \partial_{x} H_{s} \\
f_{4} \partial_{z}\left(C^{\mathrm{uvw}} \partial_{z} v\right)+f_{4} f_{0} \partial_{z}\left(C^{\mathrm{uvw}} \partial_{y} w\right)+4 f_{5} \partial_{y}\left(C^{\mathrm{uvw}} \partial_{y} v\right) & \\
+2 f_{5} \partial_{y}\left(C^{\mathrm{uvw}} \partial_{x} u\right)+f_{6} \partial_{x}\left(C^{\mathrm{uvw}}\left(\partial_{y} u+\partial_{x} v\right)\right) & =2 \rho_{\mathrm{ice}} g \partial_{y} H_{s} \\
\partial_{x} u+\partial_{y} v+\partial_{z} w & =0
\end{aligned}
$$

A numerical ice sheet

- ice shelf 3D

velocity field solution

T. J. Reerink et al.

\section{Title Page}

\section{Abstract}

Introduction

Conclusions

Tables

References

Figures

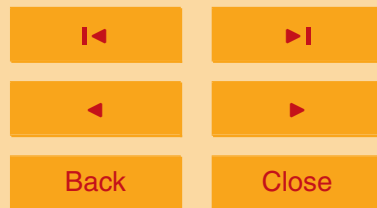

Full Screen / Esc

Printer-friendly Version 
We work out the derivatives (product rule):

$f_{4} C^{\mathrm{uvw}} \partial_{z z}^{2} u+\quad f_{0} f_{4} C^{\mathrm{uvw}} \partial_{z x}^{2} w+4 f_{5} C^{\mathrm{uvw}} \partial_{x x}^{2} u+$

$2 f_{5} C^{\mathrm{uvw}} \partial_{x y}^{2} v+f_{6} C^{\mathrm{uvw}}\left(\partial_{y y}^{2} u+\partial_{x y}^{2} v\right)+$

$f_{4}\left(\partial_{z} C^{\mathrm{uvw}}\right)\left(\partial_{z} u\right)+f_{0} f_{4}\left(\partial_{z} C^{\mathrm{uvw}}\right)\left(\partial_{x} w\right)+4 f_{5}\left(\partial_{x} C^{\mathrm{uvw}}\right)\left(\partial_{x} u\right)+$ $2 f_{5}\left(\partial_{x} C^{\mathrm{uvw}}\right)\left(\partial_{y} v\right)+f_{6}\left(\partial_{y} C^{\mathrm{uvw}}\right)\left(\partial_{y} u+\partial_{x} v\right)+$

$f_{4} C^{\mathrm{uvw}} \partial_{z z}^{2} v+$

$f_{0} f_{4} C^{\mathrm{uvw}} \partial_{z y}^{2} w+$

$2 f_{5} C^{\mathrm{uvw}} \partial_{x y}^{2} u+f_{6} C^{\mathrm{uvw}}\left(\partial_{x x}^{2} v+\partial_{x y}^{2} u\right)+$

$f_{4}\left(\partial_{z} C^{\mathrm{uvw}}\right)\left(\partial_{z} v\right)+$

$f_{0} f_{4}\left(\partial_{z} C^{u v w}\right)\left(\partial_{y} w\right)+4 f_{5}$
$\left.\partial_{x} C^{u v w}\right)\left(\partial_{y} u+\partial_{x} v\right)+$

$2 f_{5}\left(\partial_{y} C^{\mathrm{uvw}}\right)\left(\partial_{x} u\right)+f_{6}\left(\partial_{x} C^{\mathrm{uvw}}\right)\left(\partial_{y} u+\partial_{x} v\right)+$

$$
\partial_{x} u+\partial_{y} v+\partial_{z} w=0
$$

$4 f_{5} C^{\mathrm{uvw}} \partial_{y y}^{2} v+$

$+$

$4 f_{5}\left(\partial_{y} C^{\mathrm{uvw}}\right)\left(\partial_{y} v\right)+$
5 Recombining the terms and changing to the index-derivative notation (e.g. $W_{x}=\partial_{x} w$, $U_{y y}=\partial_{y y}^{2} u, C_{z}^{u v w}=\partial_{z} C^{\mathrm{uvw}}$, etc.), we get:

$$
f_{4} U_{z z}+f_{0} f_{4} W_{z x}+4 f_{5} U_{x x}+f_{6} U_{y y}+f_{56} V_{x y}
$$

$+\frac{1}{C^{\mathrm{uvw}}}\left[f_{4} C_{z}^{u v w}\left(U_{z}+f_{0} W_{x}\right)+f_{5} C_{x}^{u v w}\left(4 U_{x}+2 V_{y}\right)+f_{6} C_{y}^{u v w}\left(U_{y}+V_{x}\right)\right]=\frac{2 \rho_{\mathrm{ice}} g}{C^{\mathrm{uvw}}} \partial_{x} H_{s}$

$$
f_{4} V_{z z}+f_{0} f_{4} W_{z y}+4 f_{5} V_{y y}+f_{6} V_{x x}+f_{56} U_{x y}
$$

$10+\frac{1}{C^{u v w}}\left[f_{4} C_{z}^{u v w}\left(V_{z}+f_{0} W_{y}\right)+f_{5} C_{y}^{u v w}\left(4 V_{y}+2 U_{x}\right)+f_{6} C_{x}^{u v w}\left(U_{y}+V_{x}\right)\right]=\frac{2 \rho_{\text {ice }} g}{C^{u v w}} \partial_{y} H_{s}$

$$
U_{x}+V_{y}+W_{z}=0
$$

with

$f_{56} \equiv 2 f_{5}+f_{6}$

Equations (D10-D12) have to be transformed to the vertically scaled coordinates $x^{\prime}, y^{\prime}$

and $\zeta$. See Table 3 in Appendix B for the transformation rules for the derivative operators. Due to the vertical scaling at each grid point separately, the transformed $x$ and $y$

\section{GMDD}

2, 81-158, 2009

A numerical ice sheet - ice shelf 3D

velocity field solution

T. J. Reerink et al.

Title Page

Abstract

Introduction

Conclusions

Tables

References

Figures

14

$\rightarrow$

4

Back

Close

Full Screen / Esc

Printer-friendly Version

Interactive Discussion 
derivatives also cause correction terms. Because the scaled $x^{\prime}$ and $y^{\prime}$ coordinates are equal to the $x$ and $y$ coordinates, the accents are omitted from now on. Accomplishing the transformation gives

$$
\begin{array}{r}
f_{4} \zeta_{z}^{2} U_{\zeta \zeta}+f_{0} f_{4}\left(\zeta_{z} W_{x \zeta}+\zeta_{x} \zeta_{z} W_{\zeta \zeta}+\zeta_{x z} W_{\zeta}\right)+4 f_{5}\left(U_{x x}+2 \zeta_{x} U_{x \zeta}+\zeta_{x}^{2} U_{\zeta \zeta}+\zeta_{x x} U_{\zeta}\right) \\
+f_{6}\left(U_{y y}+2 \zeta_{y} U_{y \zeta}+\zeta_{y}^{2} U_{\zeta \zeta}+\zeta_{y y} U_{\zeta}\right)+f_{56}\left(V_{x y}+\zeta_{y} V_{x \zeta}+\zeta_{x} V_{y \zeta}+\zeta_{x} \zeta_{y} V_{\zeta \zeta}+\zeta_{x y} V_{\zeta}\right) \\
+f_{4} C^{\zeta}\left[\zeta_{z} U_{\zeta}+f_{0}\left(W_{x}+\zeta_{x} W_{\zeta}\right)\right]+f_{5} C^{x}\left[4\left(U_{x}+\zeta_{x} U_{\zeta}\right)+2\left(V_{y}+\zeta_{y} V_{\zeta}\right)\right] \\
+f_{6} C^{y}\left(U_{y}+\zeta_{y} U_{\zeta}+V_{x}+\zeta_{x} V_{\zeta}\right)=\frac{2 \rho_{\text {ice }} g}{C^{\mathrm{uvw}}} \partial_{x} H_{s}
\end{array}
$$$$
f_{4} \zeta_{z}^{2} V_{\zeta \zeta}+f_{0} f_{4}\left(\zeta_{z} w_{y \zeta}+\zeta_{y} \zeta_{z} w_{\zeta \zeta}+\zeta_{y z} w_{\zeta}\right)+4 f_{5}\left(V_{y y}+2 \zeta_{y} V_{y \zeta}+\zeta_{y}^{2} v_{\zeta \zeta}+\zeta_{y y} v_{\zeta}\right)
$$$$
+f_{6}\left(v_{x x}+2 \zeta_{x} v_{x \zeta}+\zeta_{x}^{2} v_{\zeta \zeta}+\zeta_{x x} v_{\zeta}\right)
$$$$
+f_{56}\left(U_{x y}+\zeta_{y} U_{x \zeta}+\zeta_{x} U_{y \zeta}+\zeta_{x} \zeta_{y} U_{\zeta \zeta}+\zeta_{x y} U_{\zeta}\right)
$$$$
+f_{4} C^{\zeta}\left[\zeta_{z} V_{\zeta}+f_{0}\left(W_{y}+\zeta_{y} W_{\zeta}\right)\right]+f_{5} C^{y}\left[4\left(V_{y}+\zeta_{y} V_{\zeta}\right)+2\left(U_{x}+\zeta_{x} U_{\zeta}\right)\right]
$$$$
+f_{6} C^{x}\left(U_{y}+\zeta_{y} U_{\zeta}+V_{x}+\zeta_{x} V_{\zeta}\right)=\frac{2 \rho_{\text {ice }} g}{C^{\text {uvw }}} \partial_{y} H_{s}
$$$$
U_{x}+\zeta_{x} U_{\zeta}+V_{y}+\zeta_{y} V_{\zeta}+\zeta_{z} W_{\zeta}=0
$$

\section{GMDD}

2, 81-158, 2009

A numerical ice sheet

- ice shelf 3D

velocity field solution

T. J. Reerink et al.

Title Page

Abstract

Introduction

Conclusions

References

Tables

Figures

14

$\rightarrow 1$

4

Back

Close

Full Screen / Esc

Printer-friendly Version 
Three quantities are defined which equal the transformed derivatives of the explicit $C^{\text {uvw }}$ fields but devided by $C^{\text {uvw }}$ :

$$
\begin{aligned}
C^{x} & =\frac{C_{x}^{u v w}+\zeta_{x} C_{\zeta}^{u v w}}{C^{u v w}} \\
c^{y} & =\frac{C_{y}^{u v w}+\zeta_{y} C_{\zeta}^{u v w}}{C^{u v w}} \\
C^{\zeta} & =\frac{\zeta_{z} C_{\zeta}^{u v w}}{C^{u v w}}
\end{aligned}
$$

Here, $C^{\text {uvw }}$ has to be expressed in the scaled coordinates. Equation (4.4) shows that $C^{\text {uvw }}$ contains many derivatives which have to be transformed as well. Substituting in 10 Eq. (4.4) the transformation rules for the derivatives results in

$$
\begin{aligned}
C^{\mathrm{uvw}}(x, y, \zeta)=A_{\mathrm{flow}} \mathrm{-1}^{\frac{-1}{n}}( & f_{1}\left(\partial_{x} u+\zeta_{\mathrm{x}} \partial_{\zeta} u\right)^{2}+f_{1}\left(\partial_{y} v+\zeta_{\mathrm{y}} \partial_{\zeta} v\right)^{2}+ \\
& f_{1}\left(\partial_{x} u+\zeta_{x} \partial_{\zeta} u\right)\left(\partial_{y} v+\zeta_{\mathrm{y}} \partial_{\zeta} v\right) \\
& +\frac{1}{4}\left[f_{2}\left(\partial_{y} u+\zeta_{\mathrm{y}} \partial_{\zeta} u+\partial_{x} v+\zeta_{x} \partial_{\zeta} v\right)^{2}+\right. \\
& f_{3}\left(\zeta_{z} \partial_{\zeta} u+f_{0}\left(\partial_{x} w+\zeta_{x} \partial_{\zeta} w\right)\right)^{2}+ \\
& \left.\left.f_{3}\left(\zeta_{z} \partial_{\zeta} v+f_{0}\left(\partial_{y} w+\zeta_{y} \partial_{\zeta} w\right)\right)^{2}\right]+\dot{\epsilon}_{0}^{2}\right)^{\frac{1-n}{2 n}}
\end{aligned}
$$

2, 81-158, 2009

A numerical ice sheet - ice shelf 3D velocity field solution

T. J. Reerink et al.

Title Page

\section{Abstract}

Conclusions

Tables

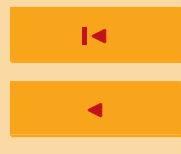

Back
Introduction

References

Figures

$\rightarrow 1$

$\rightarrow$

Close

Full Screen / Esc

In which $A_{\text {flow }}$ depends on the spacial dependent temperature $T(x, y, \zeta)$. Note that the $u, v$, and $w$ are velocities relative to the scaled coordinates. That corresponds with how they are stored in a usual ice model code. 
The Eqs. (D14-D16) are reordered on the derivatives of the variables $u, v$, and $w$ :

$4 f_{5} C^{x} U_{x}+f_{6} C^{y} U_{y}+\left(4 f_{5} C^{x} \zeta_{x}+f_{6} C^{y} \zeta_{y}+f_{4} C^{\zeta} \zeta_{z}+4 f_{5} \zeta_{x x}+f_{6} \zeta_{y y}\right) U_{\zeta}$

GMDD

$+4 f_{5} U_{x x}+8 f_{5} \zeta_{x} U_{x \zeta}+f_{6} U_{y y}$

$+2 f_{6} \zeta_{y} U_{y \zeta}+\left(4 f_{5} \zeta_{x}^{2}+f_{6} \zeta_{y}^{2}+f_{4} \zeta_{z}^{2}\right) U_{\zeta \zeta}+f_{6} C^{y} V_{x}$

$5+2 f_{5} C^{x} V_{y}+\left(f_{6} C^{y} \zeta_{x}+2 f_{5} C^{x} \zeta_{y}+f_{56} \zeta_{x y}\right) V_{\zeta}+f_{56} V_{x y}$

$+f_{56} \zeta_{y} V_{x \zeta}+f_{56} \zeta_{x} V_{y \zeta}+f_{56} \zeta_{x} \zeta_{y} V_{\zeta \zeta}+f_{0} f_{4} C^{\zeta} w_{x}+\left(f_{0} f_{4} C^{\zeta} \zeta_{x}+f_{0} f_{4} \zeta_{x z}\right) w_{\zeta}$

$+f_{0} f_{4} \zeta_{z} W_{x \zeta}+f_{0} f_{4} \zeta_{x} \zeta_{z} W_{\zeta \zeta}=\frac{2 \rho_{\text {ice }} g}{C^{\mathrm{uvw}}} \partial_{x} H_{s}$

$2 f_{5} C^{y} U_{x}+f_{6} C^{x} U_{y}+\left(2 f_{5} C^{y} \zeta_{x}+f_{6} C^{x} \zeta_{y}+f_{56} \zeta_{x y}\right) U_{\zeta}+f_{56} U_{x y}+f_{56} \zeta_{y} U_{x \zeta}$

$+f_{56} \zeta_{x} U_{y \zeta}+f_{56} \zeta_{x} \zeta_{y} U_{\zeta \zeta}$

$+f_{6} C^{x} V_{x}+4 f_{5} C^{y} V_{y}+\left(f_{6} C^{x} \zeta_{x}+4 f_{5} C^{y} \zeta_{y}+f_{4} C^{\zeta} \zeta_{z}+f_{6} \zeta_{x x}+4 f_{5} \zeta_{y y}\right) V_{\zeta}$

$+f_{6} V_{x x}+2 f_{6} \zeta_{x} V_{x \zeta}+4 f_{5} V_{y y}+8 f_{5} \zeta_{y} V_{y \zeta}$

$+\left(f_{6} \zeta_{x}^{2}+4 f_{5} \zeta_{y}^{2}+f_{4} \zeta_{z}^{2}\right) V_{\zeta \zeta}+f_{0} f_{4} C^{\zeta} W_{y}+\left(f_{0} f_{4} C^{\zeta} \zeta_{y}+f_{0} f_{4} \zeta_{y z}\right) w_{\zeta}$

$+f_{0} f_{4} \zeta_{z} W_{y \zeta}+f_{0} f_{4} \zeta_{y} \zeta_{z} W_{\zeta \zeta}=\frac{2 \rho_{\text {ice }} g}{C^{\mathrm{uvw}}} \partial_{y} H_{s}$

$U_{x}+\zeta_{x} U_{\zeta}+V_{y}+\zeta_{y} V_{\zeta}+\zeta_{z} W_{\zeta}=0$

2, 81-158, 2009

A numerical ice sheet

- ice shelf 3D

velocity field solution

T. J. Reerink et al.

Title Page

Abstract

Introduction

Conclusions

References

Tables

Figures

14

$\rightarrow 1$

4

Back

Close

Full Screen / Esc

The Eqs. (D21-D23) are shortened by defining coefficients (see Table 5) which replace

Printer-friendly Version

Interactive Discussion 
the factors in front of the discretized variables:

$$
\begin{aligned}
& f_{5}^{U_{x}} U_{x}+f_{6}^{U_{y}} U_{y}+f_{456}^{U_{\zeta}} U_{\zeta}+f_{5}^{U_{x x}} U_{x x}+f_{5}^{U_{x \zeta}} U_{x \zeta}+f_{v_{\zeta \zeta}}^{U_{y \zeta}} U_{y y}+f_{v_{y \zeta}}^{U_{y \zeta}} U_{y \zeta}+ \\
& f_{456}^{U_{\zeta \zeta}} U_{\zeta \zeta}+f_{6}^{V_{x}} V_{x}+f_{5}^{V_{y}} V_{y}+f_{56}^{V_{\zeta \zeta}} v_{\zeta}+f_{56}^{V_{x y}} V_{x y}+f_{56}^{V_{x \zeta}} V_{x \zeta}+f_{56}^{V_{y \zeta}} V_{y \zeta}+ \\
& f_{56}^{V_{\zeta \zeta}} V_{\zeta \zeta}+f_{04}^{W_{x}} W_{x}+f_{04}^{W_{\zeta}} W_{\zeta}+f_{04}^{W_{x \zeta}} W_{x \zeta}+f_{04}^{W_{\zeta \zeta}} W_{\zeta \zeta}=\frac{2 \rho_{\text {ice }} g}{C^{\mathrm{UVW}}} \partial_{x} H_{s} \\
& g_{5}^{U_{x}} U_{x}+g_{6}^{U_{y}} U_{y}+g_{56}^{U_{\zeta}} U_{\zeta}+g_{56}^{U_{x y}} U_{x y}+g_{56}^{U_{x \zeta}} U_{x \zeta}+g_{56}^{U_{y \zeta}} U_{y \zeta}+g_{56}^{U_{\zeta \zeta}} U_{\zeta \zeta}+ \\
& g_{6}^{V_{x}} v_{x}+g_{5}^{V_{y}} v_{y}+g_{456}^{V_{\zeta \zeta}} v_{\zeta}+g_{W_{y \zeta}}^{V_{x x}} v_{x x}+g_{W_{\zeta \zeta}} v_{x \zeta}+g_{5}^{V_{y y}} v_{y y}+g_{5}^{V_{y \zeta}} v_{y \zeta}+ \\
& g_{456}^{V_{\zeta \zeta}} V_{\zeta \zeta}+g_{04}^{W_{y}} W_{y}+g_{04}^{W_{\zeta}} W_{\zeta}+g_{04}^{W_{y \zeta}} W_{y \zeta}+g_{04}^{W_{\zeta \zeta}} W_{\zeta \zeta}=\frac{2 \rho_{\text {ice }} g}{C^{\mathrm{uVW}}} \partial_{y} H_{s}
\end{aligned}
$$

$$
U_{x}+\zeta_{x} U_{\zeta}+V_{y}+\zeta_{y} V_{\zeta}+\zeta_{z} W_{\zeta}=0
$$

The derivatives of $u, v$, and $w$ in Eqs. (D24-D26) are discretized with use of the finite difference rules from Table 4 in Appendix C:

\section{GMDD}

2, 81-158, 2009

A numerical ice sheet - ice shelf 3D velocity field solution

T. J. Reerink et al.

\section{Title Page}

\section{Abstract}

Introduction

Conclusions

References

Tables

Figures

14

$\rightarrow$

4

Back

\section{Full Screen / Esc}

\section{Printer-friendly Version}

Interactive Discussion 
$f_{5}^{U_{x}}\left(a_{x} U_{\mathrm{i}-1, \mathrm{j}}^{\mathrm{k}}+c_{x} U_{\mathrm{i}+1, \mathrm{j}}^{\mathrm{k}}\right)+f_{6}^{U_{y}}\left(a_{y} U_{\mathrm{i}, \mathrm{j}-1}^{\mathrm{k}}+c_{y} U_{\mathrm{i}, \mathrm{j}+1}^{\mathrm{k}}\right)$

$+\left(f_{456}^{U_{\zeta}}\right)\left(a_{\zeta} U_{\mathrm{i}, \mathrm{j}}^{\mathrm{k}-1}+b_{\zeta} U_{\mathrm{i}, \mathrm{j}}^{\mathrm{k}}+c_{\zeta} U_{\mathrm{i}, \mathrm{j}}^{\mathrm{k}+1}\right)+f_{5}^{U_{x x}}\left(a_{x x} U_{\mathrm{i}-1, \mathrm{j}}^{\mathrm{k}}+b_{x x} U_{\mathrm{i}, \mathrm{j}}^{\mathrm{k}}+c_{x x} U_{\mathrm{i}+1, \mathrm{j}}^{\mathrm{k}}\right)$

$+f_{5}^{U_{x \zeta}}\left(a_{\zeta} a_{x} U_{\mathrm{i}-1, \mathrm{j}}^{\mathrm{k}-1}+a_{\zeta} c_{x} U_{\mathrm{i}+1, \mathrm{j}}^{\mathrm{k}-1}+b_{\zeta} a_{x} U_{\mathrm{i}-1, \mathrm{j}}^{\mathrm{k}}+b_{\zeta} c_{x} U_{\mathrm{i}+1, \mathrm{j}}^{\mathrm{k}}+c_{\zeta} a_{x} U_{\mathrm{i}-1, \mathrm{j}}^{\mathrm{k}+1}+c_{\zeta} c_{x} U_{\mathrm{i}+1, \mathrm{j}}^{\mathrm{k}+1}\right)$

$+f_{6}^{U_{y y}}\left(a_{y y} U_{\mathrm{i}, \mathrm{j}-1}^{\mathrm{k}}+b_{y y} U_{\mathrm{i}, \mathrm{j}}^{\mathrm{k}}+c_{y y} U_{\mathrm{i}, \mathrm{j}+1}^{\mathrm{k}}\right)$

$+f_{6}^{U_{y \zeta}}\left(a_{\zeta} a_{y} U_{\mathrm{i}, \mathrm{j}-1}^{\mathrm{k}-1}+a_{\zeta} c_{y} U_{\mathrm{i}, \mathrm{j}+1}^{\mathrm{k}-1}+b_{\zeta} a_{y} U_{\mathrm{i}, \mathrm{j}-1}^{\mathrm{k}}+b_{\zeta} c_{y} U_{\mathrm{i}, \mathrm{j}+1}^{\mathrm{k}}+c_{\zeta} a_{y} U_{\mathrm{i}, \mathrm{j}-1}^{\mathrm{k}+1}+c_{\zeta} c_{y} U_{\mathrm{i}, \mathrm{j}+1}^{\mathrm{k}+1}\right)$

$+\left(f_{456}^{U_{\zeta \zeta}}\right)\left(a_{\zeta \zeta} U_{\mathrm{i}, \mathrm{j}}^{\mathrm{k}-1}+b_{\zeta \zeta} U_{\mathrm{i}, \mathrm{j}}^{\mathrm{k}}+c_{\zeta \zeta} U_{\mathrm{i}, \mathrm{j}}^{\mathrm{k}+1}\right)$

$+f_{6}^{V_{x}}\left(a_{x} V_{\mathrm{i}-1, \mathrm{j}}^{\mathrm{k}}+c_{x} V_{\mathrm{i}+1, \mathrm{j}}^{\mathrm{k}}\right)+f_{5}^{V_{y}}\left(a_{y} V_{\mathrm{i}, \mathrm{j}-1}^{\mathrm{k}}+c_{y} V_{\mathrm{i}, \mathrm{j}+1}^{\mathrm{k}}\right)$

$+\left(f_{56}^{V_{\zeta}}\right)\left(a_{\zeta} V_{\mathrm{i}, \mathrm{j}}^{\mathrm{k}-1}+b_{\zeta} V_{\mathrm{i}, \mathrm{j}}^{\mathrm{k}}+c_{\zeta} V_{\mathrm{i}, \mathrm{j}}^{\mathrm{k}+1}\right)$

$+f_{56}^{V_{x y}}\left(a_{x} a_{y} V_{\mathrm{i}-1, \mathrm{j}-1}^{\mathrm{k}}+a_{x} c_{y} V_{\mathrm{i}-1, \mathrm{j}+1}^{\mathrm{k}}+c_{x} a_{y} V_{\mathrm{i}+1, \mathrm{j}-1}^{\mathrm{k}}+c_{x} c_{y} V_{\mathrm{i}+1, \mathrm{j}+1}^{\mathrm{k}}\right)$

$+f_{56}^{V_{x \zeta}}\left(a_{\zeta} a_{x} V_{\mathrm{i}-1, \mathrm{j}}^{\mathrm{k}-1}+a_{\zeta} c_{x} V_{\mathrm{i}+1, \mathrm{j}}^{\mathrm{k}-1}+b_{\zeta} a_{x} V_{\mathrm{i}-1, \mathrm{j}}^{\mathrm{k}}+b_{\zeta} c_{x} V_{\mathrm{i}+1, \mathrm{j}}^{\mathrm{k}}+c_{\zeta} a_{x} V_{\mathrm{i}-1, \mathrm{j}}^{\mathrm{k}+1}+c_{\zeta} c_{x} V_{\mathrm{i}+1, \mathrm{j}}^{\mathrm{k}+1}\right)$

$+f_{56}^{V_{y \zeta}}\left(a_{\zeta} a_{y} V_{\mathrm{i}, \mathrm{j}-1}^{\mathrm{k}-1}+a_{\zeta} c_{y} V_{\mathrm{i}, \mathrm{j}+1}^{\mathrm{k}-1}+b_{\zeta} a_{y} V_{\mathrm{i}, \mathrm{j}-1}^{\mathrm{k}}+b_{\zeta} c_{y} V_{\mathrm{i}, \mathrm{j}+1}^{\mathrm{k}}+c_{\zeta} a_{y} V_{\mathrm{i}, \mathrm{j}-1}^{\mathrm{k}+1}+c_{\zeta} c_{y} V_{\mathrm{i}, \mathrm{j}+1}^{\mathrm{k}+1}\right)$

$+f_{56}^{V_{\zeta \zeta}}\left(a_{\zeta \zeta} V_{\mathrm{i}, \mathrm{j}}^{\mathrm{k}-1}+b_{\zeta \zeta} V_{\mathrm{i}, \mathrm{j}}^{\mathrm{k}}+c_{\zeta \zeta} V_{\mathrm{i}, \mathrm{j}}^{\mathrm{k}+1}\right)$

$+f_{04}^{W_{x}}\left(a_{x} W_{\mathrm{i}-1, \mathrm{j}}^{\mathrm{k}}+c_{x} W_{\mathrm{i}+1, \mathrm{j}}^{\mathrm{k}}\right)+\left(f_{04}^{W_{\zeta}}\right)\left(a_{\zeta} W_{\mathrm{i}, \mathrm{j}}^{\mathrm{k}-1}+b_{\zeta} W_{\mathrm{i}, \mathrm{j}}^{\mathrm{k}}+c_{\zeta} W_{\mathrm{i}, \mathrm{j}}^{\mathrm{k}+1}\right)$

$+f_{04}^{W_{x \zeta}}\left(a_{\zeta} a_{x} W_{\mathrm{i}-1, \mathrm{j}}^{\mathrm{k}-1}+a_{\zeta} c_{x} W_{\mathrm{i}+1, \mathrm{j}}^{\mathrm{k}-1}+b_{\zeta} a_{x} W_{\mathrm{i}-1, \mathrm{j}}^{\mathrm{k}}+b_{\zeta} c_{x} W_{\mathrm{i}+1, \mathrm{j}}^{\mathrm{k}}+c_{\zeta} a_{x} W_{\mathrm{i}-1, \mathrm{j}}^{\mathrm{k}+1}+c_{\zeta} c_{x} W_{\mathrm{i}+1, \mathrm{j}}^{\mathrm{k}+1}\right)$

$+f_{04}^{W_{\zeta \zeta}}\left(a_{\zeta \zeta} W_{\mathrm{i}, \mathrm{j}}^{\mathrm{k}-1}+b_{\zeta \zeta} W_{\mathrm{i}, \mathrm{j}}^{\mathrm{k}}+c_{\zeta \zeta} W_{\mathrm{i}, \mathrm{j}}^{\mathrm{k}+1}\right)=\frac{2 \rho_{\mathrm{ice}} g}{c^{\mathrm{uvw}}} \partial_{x} H_{s}$
GMDD

2, 81-158, 2009

A numerical ice sheet

- ice shelf 3D

velocity field solution

T. J. Reerink et al.

Title Page

Abstract

Introduction

Conclusions

Tables

References

Figures

14

I

4

Back

Close

Full Screen / Esc

Printer-friendly Version

Interactive Discussion 
$g_{5}^{U_{x}}\left(a_{x} U_{\mathrm{i}-1, \mathrm{j}}^{\mathrm{k}}+c_{x} U_{\mathrm{i}+1, \mathrm{j}}^{\mathrm{k}}\right)+g_{6}^{U_{y}}\left(a_{y} U_{\mathrm{i}, \mathrm{j}-1}^{\mathrm{k}}+c_{y} U_{\mathrm{i}, \mathrm{j}+1}^{\mathrm{k}}\right)$

GMDD

$+\left(g_{56}^{U_{\zeta}}\right)\left(a_{\zeta} U_{\mathrm{i}, \mathrm{j}}^{\mathrm{k}-1}+b_{\zeta} U_{\mathrm{i}, \mathrm{j}}^{\mathrm{k}}+c_{\zeta} U_{\mathrm{i}, \mathrm{j}}^{\mathrm{k}+1}\right)$

2, 81-158, 2009

$+g_{56}^{U_{x y}}\left(a_{x} a_{y} U_{\mathrm{i}-1, \mathrm{j}-1}^{\mathrm{k}}+a_{x} c_{y} U_{\mathrm{i}-1, \mathrm{j}+1}^{\mathrm{k}}+c_{x} a_{y} U_{\mathrm{i}+1, \mathrm{j}-1}^{\mathrm{k}}+c_{x} c_{y} U_{\mathrm{i}+1, \mathrm{j}+1}^{\mathrm{k}}\right)$

$+g_{56}^{U_{x \zeta}}\left(a_{\zeta} a_{x} U_{\mathrm{i}-1, \mathrm{j}}^{\mathrm{k}-1}+a_{\zeta} c_{x} U_{\mathrm{i}+1, \mathrm{j}}^{\mathrm{k}-1}+b_{\zeta} a_{x} U_{\mathrm{i}-1, \mathrm{j}}^{\mathrm{k}}+b_{\zeta} c_{x} U_{\mathrm{i}+1, \mathrm{j}}^{\mathrm{k}}+c_{\zeta} a_{x} U_{\mathrm{i}-1, \mathrm{j}}^{\mathrm{k}+1}+c_{\zeta} c_{x} U_{\mathrm{i}+1, \mathrm{j}}^{\mathrm{k}+1}\right)$

$+g_{56}^{U_{y \zeta}}\left(a_{\zeta} a_{y} U_{\mathrm{i}, \mathrm{j}-1}^{\mathrm{k}-1}+a_{\zeta} c_{y} U_{\mathrm{i}, \mathrm{j}+1}^{\mathrm{k}-1}+b_{\zeta} a_{y} U_{\mathrm{i}, \mathrm{j}-1}^{\mathrm{k}}+b_{\zeta} c_{y} U_{\mathrm{i}, \mathrm{j}+1}^{\mathrm{k}}+c_{\zeta} a_{y} U_{\mathrm{i}, \mathrm{j}-1}^{\mathrm{k}+1}+c_{\zeta} c_{y} U_{\mathrm{i}, \mathrm{j}+1}^{\mathrm{k}+1}\right)$

$+g_{56}^{U_{\zeta \zeta}}\left(a_{\zeta \zeta} U_{\mathrm{i}, \mathrm{j}}^{\mathrm{k}-1}+b_{\zeta \zeta} U_{\mathrm{i}, \mathrm{j}}^{\mathrm{k}}+c_{\zeta \zeta} U_{\mathrm{i}, \mathrm{j}}^{\mathrm{k}+1}\right)$

$+g_{6}^{V_{x}}\left(a_{x} V_{\mathrm{i}-1, \mathrm{j}}^{\mathrm{k}}+c_{x} V_{\mathrm{i}+1, \mathrm{j}}^{\mathrm{k}}\right)+g_{5}^{V_{y}}\left(a_{y} V_{\mathrm{i}, \mathrm{j}-1}^{\mathrm{k}}+c_{y} V_{\mathrm{i}, \mathrm{j}+1}^{\mathrm{k}}\right)$

$+\left(g_{456}^{V_{\zeta}}\right)\left(a_{\zeta} V_{\mathrm{i}, \mathrm{j}}^{\mathrm{k}-1}+b_{\zeta} V_{\mathrm{i}, \mathrm{j}}^{\mathrm{k}}+c_{\zeta} V_{\mathrm{i}, \mathrm{j}}^{\mathrm{k}+1}\right)$

$+g_{6}^{V_{x x}}\left(a_{x x} V_{\mathrm{i}-1, \mathrm{j}}^{\mathrm{k}}+b_{x x} V_{\mathrm{i}, \mathrm{j}}^{\mathrm{k}}+c_{x x} V_{\mathrm{i}+1, \mathrm{j}}^{\mathrm{k}}\right)$

$+g_{6}^{V_{x \zeta}}\left(a_{\zeta} a_{x} V_{\mathrm{i}-1, \mathrm{j}}^{\mathrm{k}-1}+a_{\zeta} c_{x} V_{\mathrm{i}+1, \mathrm{j}}^{\mathrm{k}-1}+b_{\zeta} a_{x} V_{\mathrm{i}-1, \mathrm{j}}^{\mathrm{k}}+b_{\zeta} c_{x} V_{\mathrm{i}+1, \mathrm{j}}^{\mathrm{k}}+c_{\zeta} a_{x} V_{\mathrm{i}-1, \mathrm{j}}^{\mathrm{k}+1}+c_{\zeta} c_{x} V_{\mathrm{i}+1, \mathrm{j}}^{\mathrm{k}+1}\right)$

$+g_{5}^{V_{y y}}\left(a_{y y} V_{\mathrm{i}, \mathrm{j}-1}^{\mathrm{k}}+b_{y y} V_{\mathrm{i}, \mathrm{j}}^{\mathrm{k}}+c_{y y} V_{\mathrm{i}, \mathrm{j}+1}^{\mathrm{k}}\right)$

$+g_{5}^{V_{y \zeta}}\left(a_{\zeta} a_{y} V_{\mathrm{i}, \mathrm{j}-1}^{\mathrm{k}-1}+a_{\zeta} c_{y} V_{\mathrm{i}, \mathrm{j}+1}^{\mathrm{k}-1}+b_{\zeta} a_{y} V_{\mathrm{i}, \mathrm{j}-1}^{\mathrm{k}}+b_{\zeta} c_{y} V_{\mathrm{i}, \mathrm{j}+1}^{\mathrm{k}}+c_{\zeta} a_{y} V_{\mathrm{i}, \mathrm{j}-1}^{\mathrm{k}+1}+c_{\zeta} c_{y} V_{\mathrm{i}, \mathrm{j}+1}^{\mathrm{k}+1}\right)$

$+\left(g_{456}^{V_{\zeta \zeta}}\right)\left(a_{\zeta \zeta} V_{\mathrm{i}, \mathrm{j}}^{\mathrm{k}-1}+b_{\zeta \zeta} V_{\mathrm{i}, \mathrm{j}}^{\mathrm{k}}+c_{\zeta \zeta} V_{\mathrm{i}, \mathrm{j}}^{\mathrm{k}+1}\right)+g_{04}^{W_{y}}\left(a_{y} W_{\mathrm{i}, \mathrm{j}-1}^{\mathrm{k}}+c_{y} W_{\mathrm{i}, \mathrm{j}+1}^{\mathrm{k}}\right)$

Back

Title Page

Abstract

Introduction

Conclusions

References

Tables

Figures

$+\left(g_{04}^{W_{\zeta}}\right)\left(a_{\zeta} W_{\mathrm{i}, \mathrm{j}}^{\mathrm{k}-1}+b_{\zeta} W_{\mathrm{i}, \mathrm{j}}^{\mathrm{k}}+c_{\zeta} W_{\mathrm{i}, \mathrm{j}}^{\mathrm{k}+1}\right)$

$+g_{04}^{W_{y \zeta}}\left(a_{\zeta} a_{y} W_{\mathrm{i}, \mathrm{j}-1}^{\mathrm{k}-1}+a_{\zeta} c_{y} W_{\mathrm{i}, \mathrm{j}+1}^{\mathrm{k}-1}+b_{\zeta} a_{y} W_{\mathrm{i}, \mathrm{j}-1}^{\mathrm{k}}+b_{\zeta} c_{y} W_{\mathrm{i}, \mathrm{j}+1}^{\mathrm{k}}+c_{\zeta} a_{y} W_{\mathrm{i}, \mathrm{j}-1}^{\mathrm{k}+1}+c_{\zeta} c_{y} W_{\mathrm{i}, \mathrm{j}+1}^{\mathrm{k}+1}\right)$

14

I

$+g_{04}^{W_{\zeta \zeta}}\left(a_{\zeta \zeta} W_{\mathrm{i}, \mathrm{j}}^{\mathrm{k}-1}+b_{\zeta \zeta} W_{\mathrm{i}, \mathrm{j}}^{\mathrm{k}}+c_{\zeta \zeta} W_{\mathrm{i}, \mathrm{j}}^{\mathrm{k}+1}\right)=\frac{2 \rho_{\mathrm{ice}} g}{C^{\mathrm{uvw}}} \partial_{y} H_{s}$

(D28)

\section{Full Screen / Esc}

Printer-friendly Version

Interactive Discussion 


$$
\begin{aligned}
& \left(a_{x} U_{i-1, j}^{k}+c_{x} U_{i+1, j}^{k}\right)+\zeta_{x}\left(a_{\zeta} U_{i, j}^{k-1}+b_{\zeta} U_{i, j}^{k}+c_{\zeta} U_{i, j}^{k+1}\right)+\left(a_{y} V_{i, j-1}^{k}+c_{y} V_{i, j+1}^{k}\right) \\
+ & \zeta_{y}\left(a_{\zeta} V_{i, j}^{k-1}+b_{\zeta} V_{i, j}^{k}+c_{\zeta} V_{i, j}^{k+1}\right)+\zeta_{z}\left(a_{\zeta} W_{i, j}^{k-1}+b_{\zeta} W_{i, j}^{k}+c_{\zeta} W_{i, j}^{k+1}\right)=0
\end{aligned}
$$

Equations (D27-D29) form the discretized set of equations for a non-equidistant vertical grid. The non-equidistant discretization coefficients are given in Table 4. Defining 5 the many coefficients which contain the factors in front of the discretized variables, we get (in same order)

A numerical ice sheet - ice shelf 3D velocity field solution

T. J. Reerink et al.

\section{Title Page}

\section{Abstract}

Introduction

Conclusions

References

Tables

Figures

14 $>$ I

4

Back

Full Screen / Esc

Printer-friendly Version

Interactive Discussion 
$a_{\left(v_{1}\right)_{j-1, j}^{k}}^{1} U_{i-1, j}^{k}+a_{\left(v_{1}\right)_{i+1, j}^{k}}^{1} U_{i+1, j}^{k}+a_{\left(v_{1}\right)_{i, j-1}^{k}}^{1} U_{i, j-1}^{k}+a_{\left(v_{1}\right)_{i, j+1}^{k}}^{1} U_{i, j+1}^{k}+a_{\left(v_{1}\right)_{i, j}^{k-1}}^{1} U_{i, j}^{k-1}$

$+a_{\left(v_{1}\right)_{i, j}^{k}}^{1} U_{i, j}^{k}+a_{\left(v_{1}\right)_{i, j}^{k+1}}^{1} U_{i, j}^{k+1}$

$+a_{\left(v_{1}\right)_{i-1, j}^{k-1}}^{1} U_{i-1, j}^{k-1}+a_{\left(v_{1}\right)_{i+1, j}^{k-1}}^{1} U_{i+1, j}^{k-1}+a_{\left(v_{1}\right)_{i-1, j}^{k+1}}^{1} U_{i-1, j}^{k+1}+a_{\left(v_{1}\right)_{i+1, j}^{k+1}}^{1} U_{i+1, j}^{k+1}+a_{\left(v_{1}\right)_{i, j-1}^{k-1}}^{1} U_{i, j-1}^{k-1}$

$+a_{\left(v_{1}\right)_{i, j+1}^{k-1}}^{1} U_{\mathrm{i}, \mathrm{j}+1}^{\mathrm{k}-1}+a_{\left(v_{1}\right)_{i, j-1}^{k+1}}^{1} U_{\mathrm{i}, \mathrm{j}-1}^{\mathrm{k}+1}+a_{\left(v_{1}\right)_{i, j+1}^{k+1}}^{1} U_{\mathrm{i}, \mathrm{j}+1}^{\mathrm{k}+1}$

$5+a_{\left(v_{2}\right)_{j-1, j}^{k}}^{1} V_{i-1, j}^{k}+a_{\left(v_{2}\right)_{i+1, j}^{k}}^{1} V_{i+1, j}^{k}+a_{\left(v_{2}\right)_{i, j-1}^{k}}^{1} V_{i, j-1}^{k}+a_{\left(v_{2}\right)_{i, j+1}^{k}}^{1} V_{i, j+1}^{k}+a_{\left(v_{2}\right)_{i, j}^{k-1}}^{1} V_{i, j}^{k-1}$

$+a_{\left(v_{2}\right)_{i, j}^{k}}^{1} V_{i, j}^{k}+a_{\left(v_{2}\right)_{i, j}^{k+1}}^{1} V_{i, j}^{k+1}$

$+a_{\left(v_{2}\right)_{j-1, j}^{k-1}}^{1} V_{i-1, j}^{k-1}+a_{\left(v_{2}\right)_{i+1, j}^{k-1}}^{1} V_{i+1, j}^{k-1}+a_{\left(v_{2}\right)_{i-1, j}^{k+1}}^{1} V_{i-1, j}^{k+1}+a_{\left(v_{2}\right)_{i+1, j}^{k+1}}^{1} V_{i+1, j}^{k+1}+a_{\left(v_{2}\right)_{i, j-1}^{k-1}}^{1} V_{i, j-1}^{k-1}$

$+a_{\left(v_{2}\right)_{i, j+1}^{k-1}}^{1} V_{i, j+1}^{k-1}$

$+a_{\left(v_{2}\right)_{i, j-1}^{k+1}}^{1} V_{i, j-1}^{\mathrm{k}+1}+a_{\left(v_{2}\right)_{i, j+1}^{k+1}}^{1} V_{\mathrm{i}, \mathrm{j}+1}^{\mathrm{k}+1}+a_{\left(\mathrm{v}_{2}\right)_{i-1, j-1}^{k}}^{1} V_{\mathrm{i}-1, \mathrm{j}-1}^{\mathrm{k}}+a_{\left(\mathrm{v}_{2}\right)_{i-1, j+1}^{k}}^{1} V_{\mathrm{i}-1, \mathrm{j}+1}^{\mathrm{k}}$

$+a_{\left(v_{2}\right)_{i+1, j-1}^{k}}^{1} V_{\mathrm{i}+1, \mathrm{j}-1}^{\mathrm{k}}+a_{\left(v_{2}\right)_{i+1, j+1}^{k}}^{1} V_{\mathrm{i}+1, \mathrm{j}+1}^{\mathrm{k}}$

$+a_{\left(v_{3}\right)_{i-1, j}^{k}}^{1} W_{\mathrm{i}-1, \mathrm{j}}^{\mathrm{k}}+a_{\left(\mathrm{v}_{3}\right)_{i+1, j}^{k}}^{1} W_{\mathrm{i}+1, \mathrm{j}}^{\mathrm{k}}+a_{\left(\mathrm{v}_{3}\right)_{i, j}^{k-1}}^{1} W_{\mathrm{i}, \mathrm{j}}^{\mathrm{k}-1}+a_{\left(\mathrm{v}_{3}\right)_{i, j}^{k}}^{1} W_{\mathrm{i}, \mathrm{j}}^{\mathrm{k}}+a_{\left(\mathrm{v}_{3}\right)_{i, j}^{k+1}}^{1} W_{\mathrm{i}, \mathrm{j}}^{\mathrm{k}+1}$

$+a_{\left(v_{3}\right)_{i-1, j}^{k-1}}^{1} w_{\mathrm{i}-1, \mathrm{j}}^{\mathrm{k}-1}+a_{\left(\mathrm{v}_{3}\right)_{i+1, j}^{k-1}}^{1} w_{\mathrm{i}+1, \mathrm{j}}^{\mathrm{k}-1}+a_{\left(\mathrm{v}_{3}\right)_{i-1, j}^{k+1}}^{1} W_{\mathrm{i}-1, \mathrm{j}}^{\mathrm{k}+1}+a_{\left(v_{3}\right)_{i+1, j}^{k+1}}^{1} w_{\mathrm{i}+1, \mathrm{j}}^{\mathrm{k}+1}=\frac{2 \rho_{\mathrm{ice}} g}{C^{\mathrm{uvw}}} \partial_{x} H_{s}$

GMDD

2, 81-158, 2009

A numerical ice sheet

- ice shelf 3D

velocity field solution

T. J. Reerink et al.

Title Page

Abstract

Introduction

Conclusions

References

Tables

Figures

14

I

4

Back

Close

Printer-friendly Version

Interactive Discussion 
$a_{\left(v_{1}\right)_{i-1, j}^{k}}^{2} U_{i-1, j}^{k}+a_{\left(v_{1}\right)_{i+1, j}^{k}}^{2} U_{i+1, j}^{k}+a_{\left(v_{1}\right)_{i, j-1}^{k}}^{2} U_{i, j-1}^{k}+a_{\left(v_{1}\right)_{i, j+1}^{k}}^{2} U_{i, j+1}^{k}+a_{\left(v_{1}\right)_{i, j}^{k-1}}^{2} U_{i, j}^{k-1}$

$+a_{\left(v_{1}\right)_{i, j}^{k}}^{2} U_{i, j}^{k}+a_{\left(v_{1}\right)_{i, j}^{k+1}}^{2} U_{i, j}^{k+1}$

$+a_{\left(v_{1}\right)_{i-1, j}^{k-1}}^{2} U_{i-1, j}^{k-1}+a_{\left(v_{1}\right)_{j+1, j}^{k-1}}^{2} U_{i+1, j}^{k-1}+a_{\left(v_{1}\right)_{i-1, j}^{k+1}}^{2} U_{i-1, j}^{k+1}+a_{\left(v_{1}\right)_{i+1, j}^{k+1}}^{2} U_{i+1, j}^{k+1}+a_{\left(v_{1}\right)_{i, j-1}^{k-1}}^{2} U_{i, j-1}^{k-1}$

$+a_{\left(v_{1}\right)_{i, j+1}^{k-1}}^{2} U_{\mathrm{i}, \mathrm{j}+1}^{\mathrm{k}-1}$

$+a_{\left(v_{1}\right)_{i, j-1}^{k+1}}^{2} U_{i, j-1}^{k+1}+a_{\left(v_{1}\right)_{i, j+1}^{k+1}}^{2} U_{i, j+1}^{k+1}+a_{\left(v_{1}\right)_{i-1, j-1}^{k}}^{2} U_{i-1, j-1}^{k}+a_{\left(v_{1}\right)_{j-1, j+1}^{k}}^{2} U_{i-1, j+1}^{k}$

$+a_{\left(v_{1}\right)_{i+1, j-1}^{k}}^{2} U_{i+1, j-1}^{k}+a_{\left(v_{1}\right)_{i+1, j+1}^{k}}^{2} U_{i+1, j+1}^{k}$

$+a_{\left(v_{2}\right)_{i-1, j}^{k}}^{2} V_{i-1, j}^{k}+a_{\left(v_{2}\right)_{i+1, j}^{k}}^{2} V_{i+1, j}^{k}+a_{\left(v_{2}\right)_{i, j-1}^{k}}^{2} V_{i, j-1}^{k}+a_{\left(v_{2}\right)_{i, j+1}^{k}}^{2} V_{i, j+1}^{k}+a_{\left(v_{2}\right)_{i, j}^{k-1}}^{2} V_{i, j}^{k-1}$

$+a_{\left(v_{2}\right)_{i, j}^{k}}^{2} V_{i, j}^{k}+a_{\left(v_{2}\right)_{i, j}^{k+1}}^{2} V_{i, j}^{k+1}$

$+a_{\left(v_{2}\right)_{j-1, j}^{k-1}}^{2} V_{\mathrm{i}-1, \mathrm{j}}^{\mathrm{k}-1}+a_{\left(v_{2}\right)_{i+1, j}^{k-1}}^{2} V_{\mathrm{i}+1, \mathrm{j}}^{\mathrm{k}-1}+a_{\left(v_{2}\right)_{i-1, j}^{k+1}}^{2} V_{\mathrm{i}-1, \mathrm{j}}^{\mathrm{k}+1}+a_{\left(v_{2}\right)_{i+1, j}^{k+1}}^{2} V_{\mathrm{i}+1, \mathrm{j}}^{\mathrm{k}+1}+a_{\left(v_{2}\right)_{i, j-1}^{k-1}}^{2} V_{\mathrm{i}, \mathrm{j}-1}^{\mathrm{k}-1}$

$+a_{\left(v_{2}\right)_{i, j+1}^{k-1}}^{2} V_{\mathrm{i}, \mathrm{j}+1}^{\mathrm{k}-1}+a_{\left(v_{2}\right)_{i, j-1}^{k+1}}^{2} V_{\mathrm{i}, \mathrm{j}-1}^{\mathrm{k}+1}+a_{\left(v_{2}\right)_{i, j+1}^{k+1}}^{2} V_{\mathrm{i}, \mathrm{j}+1}^{\mathrm{k}+1}$

$+a_{\left(v_{3}\right)_{i, j-1}^{k}}^{2} W_{i, j-1}^{k}+a_{\left(v_{3}\right)_{i, j+1}^{k}}^{2} W_{i, j+1}^{k}+a_{\left(v_{3}\right)_{i, j}^{k-1}}^{2} W_{i, j}^{k-1}+a_{\left(v_{3}\right)_{i, j}^{k}}^{2} W_{i, j}^{k}+a_{\left(v_{3}\right)_{i, j}^{k+1}}^{2} W_{i, j}^{k+1}$

$+a_{\left(v_{3}\right)_{i, j-1}^{k-1}}^{2} W_{\mathrm{i}, \mathrm{j}-1}^{\mathrm{k}-1}+a_{\left(\mathrm{v}_{3}\right)_{i, j+1}^{k-1}}^{2} W_{\mathrm{i}, \mathrm{j}+1}^{\mathrm{k}-1}+a_{\left(\mathrm{v}_{3}\right)_{i, j-1}^{k+1}}^{2} W_{\mathrm{i}, \mathrm{j}-1}^{\mathrm{k}+1}+a_{\left(\mathrm{v}_{3}\right)_{i, j+1}^{k+1}}^{2} W_{\mathrm{i}, \mathrm{j}+1}^{\mathrm{k}+1}=\frac{2 \rho_{\mathrm{ice}} g}{C^{\mathrm{uvw}}} \partial_{y} H_{s}$

(D31)

$a_{\left(v_{1}\right)_{j-1, j}^{k}}^{3} U_{i-1, j}^{k}+a_{\left(v_{1}\right)_{i+1, j}^{k}}^{3} U_{i+1, j}^{k}+a_{\left(v_{1}\right)_{i, j}^{k-1}}^{3} U_{i, j}^{k-1}+a_{\left(v_{1}\right)_{i, j}^{k}}^{3} U_{i, j}^{k}+a_{\left(v_{1}\right)_{i, j}^{k+1}}^{3} U_{i, j}^{k+1}$

$+a_{\left(v_{2}\right)_{i, j-1}^{k}}^{3} V_{i, j-1}^{\mathrm{k}}+a_{\left(v_{2}\right)_{i, j+1}^{k}}^{3} V_{\mathrm{i}, \mathrm{j}+1}^{\mathrm{k}}+a_{\left(v_{2}\right)_{i, j}^{k-1}}^{3} V_{\mathrm{i}, \mathrm{j}}^{\mathrm{k}-1}+a_{\left(v_{2}\right)_{i, j}^{k}}^{3} V_{\mathrm{i}, \mathrm{j}}^{\mathrm{k}}+a_{\left(v_{2}\right)_{i, j}^{k+1}}^{3} V_{\mathrm{i}, \mathrm{j}}^{\mathrm{k}+1}$

$+a_{\left(v_{3}\right)_{i, j}^{k-1}}^{3} W_{i, j}^{k-1}+a_{\left(v_{3}\right)_{i, j}^{k}}^{3} W_{i, j}^{k}+a_{\left(v_{3}\right)_{i, j}^{k+1}}^{3} W_{i, j}^{k+1}=0$

In their subscript these coefficients contain the name of the discretized variable where 129

\section{GMDD}

2, 81-158, 2009

A numerical ice sheet

- ice shelf 3D

velocity field solution

T. J. Reerink et al.

Title Page

Abstract

Introduction

Conclusions

Tables

References

Figures

14

$\rightarrow 1$

4

Back

Close

Full Screen / Esc

Printer-friendly Version

Interactive Discussion 
they are working on. These coefficients are defined in the Tables 6-8 in the same order. Given that $v_{1} \equiv U, v_{2} \equiv V$ and $v_{3} \equiv W$, those names in the subscript agree. The transformed boudary conditions in Sect. 5.2 can be found in Table 9 where they are applyed as Dirichlet and Neumann conditions. The correction terms due to the 5 scaling for the horizontal derivatives are neclected because the scaling is assumed to be equal for neighbour points at the domain edges. The coefficients in the matrix equation concerning those boundary conditions are presented behind each corresponding equation in Table 9. The results presented in this appendix were verified by backward substitution with help of Mathematica.

\section{Appendix E}

\section{Matrix format for $n$ coupled equations on a three or $d$ dimensional grid}

We consider a set of $n$ physical equations with an equal number of variables defined on a 3D grid which contains linear derivatives in every grid direction. If the implicit 15 taken linear derivative terms contain all the $n$ variables, then all this equations at all this grid points have to be solved coupled. The derivatives in each grid direction cause the dependence of each grid point on its neighbours in that direction because of the (central second order) finite difference schemes used to discretize the linear derivative terms implicitly. E.g. in our case in Eqs. (5.1-5.3) we take all the linear first derivatives, all the linear second derivatives, and all the linear second cross derivatives implicit. It means for $n$ physical equations on a 3D (x,y,z)-grid of size $N_{\mathrm{x}} N_{\mathrm{y}} N_{z}$, that we have to solve the $N \equiv n N_{\mathrm{x}} N_{\mathrm{y}} N_{z}$ equations at this grid simultaneously.

For a given $i j k$ grid point we call the (implicit) variables like $U_{\mathrm{i}, \mathrm{j}}^{\mathrm{k}}, V_{\mathrm{i}, \mathrm{j}}^{\mathrm{k}}, W_{\mathrm{i}, \mathrm{j}}^{\mathrm{k}}$ (or generaly $V_{1 \mathrm{i}, \mathrm{j}}^{\mathrm{k}}, \ldots, V_{n \mathrm{i}, \mathrm{j}}^{\mathrm{k}}$ ) central variables, and the (implicit) variables like $U_{\mathrm{i}-1, \mathrm{j}}^{\mathrm{k}}, V_{\mathrm{i}, \mathrm{j}+1}^{\mathrm{k}-1}$, etc. 25 neighbour variables.

We consider the matrix equation, with an $N \times N$ matrix $A$, a solution vector $v$ and a

GMDD

2, 81-158, 2009

A numerical ice sheet - ice shelf 3D velocity field solution

T. J. Reerink et al.

Title Page

Abstract

Introduction

Conclusions

Tables

References

Figures

14

$\rightarrow 1$

4

Back

Close

\section{Full Screen / Esc}

Printer-friendly Version

Interactive Discussion 
right hand side vector RHS both of length $N$,

$A v=\mathrm{RHS}$

describing a set of $n$ equations with $n$ variables $v_{1} \ldots v_{n}$ on a 3D $(\mathrm{x}, \mathrm{y}, \mathrm{z})$-grid of size $N_{\mathrm{x}} N_{\mathrm{y}} N_{z}$

5 E1 The structure of the matrix equation

All $n$ equations for all $N_{\mathrm{x}} N_{\mathrm{y}} N_{z}$ grid points have to be placed in this matrix Eq. (E1). This requires a format which organizes the position of all this equations in this matrix equation. We choose to structure the format as follows (only the matrix coefficients working on the central variables are shown, those working on the neighbour variables are indicated by the most left and the most right dots in the matrix $A^{\prime \prime}$ down):

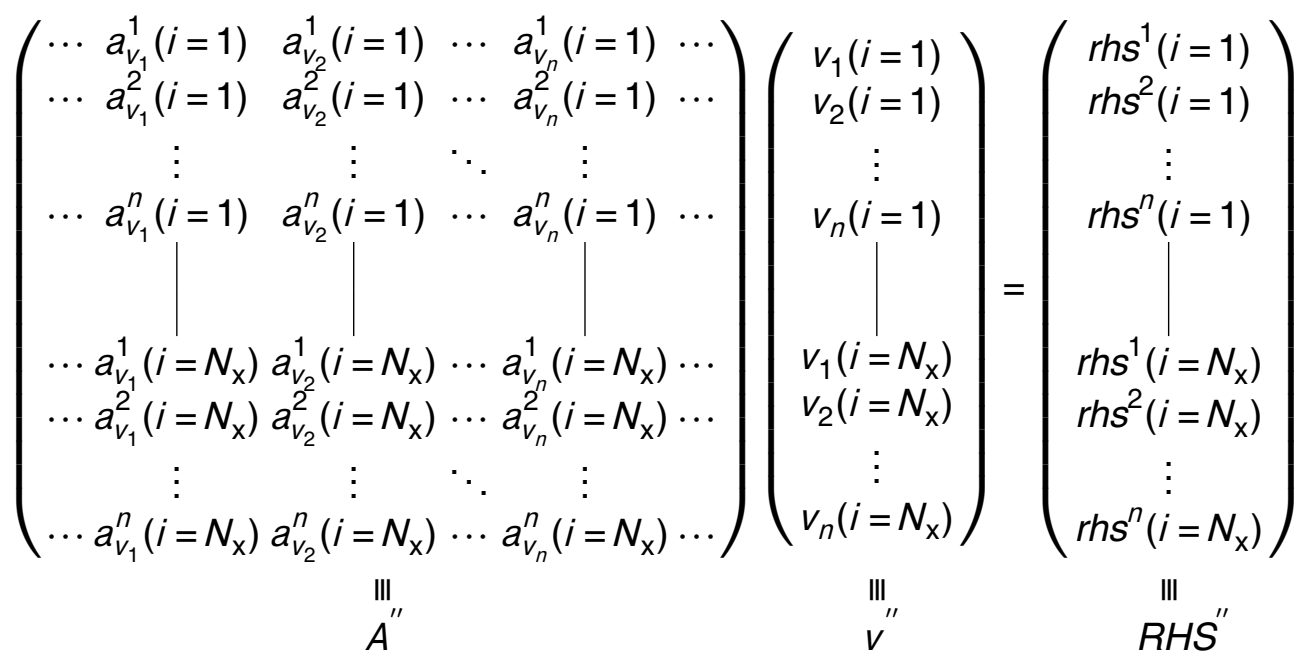

In Eq. (E2) we see how the solution vector $v^{\prime \prime}$ is organized: for each $i$ all variables ${ }_{15} v_{1} \ldots v_{n}$ are placed below each other, which is repated from $i=1$ up to $i=N_{\mathrm{x}}$. Between $i=1$ and $i=N_{\mathrm{x}}$ the variables are indicated by a vertical line.

\section{GMDD}

2, 81-158, 2009

A numerical ice sheet - ice shelf 3D velocity field solution

T. J. Reerink et al.

\section{Title Page}

\section{Abstract}

Introduction

Conclusions

Tables

References

Figures

14

$\rightarrow 1$

4

Back

Close

\section{Full Screen / Esc}

Printer-friendly Version 
The coefficients of $A^{\prime \prime}$ working on the central variables are indicated in Eq. (E2) for $i=1$ up to $i=N_{x}$, where those for the $i$ 's between are indicated by a vertical line. Each coefficient of $A$ is labeled by a subscript equal to the name of the variable it works on, and a superscript equal to the equation number it belongs to. The dependence on $i$ is 5 der $i$, the non-shown coefficients working on the neighbour variables. The $R H S^{\prime \prime}$ vector is composed analogue to the solution vector $v$ " and each component is labeled by a superscript equal to the equation number it belongs to.

With this matrix equation $A^{\prime \prime} v^{\prime \prime}=R H S^{\prime \prime}$ only the $x$-derivatives (of all $n$ variables) can be taken implicit in the discretized equations. In case we want to include the $y$-derivatives implicitly in the discretized equations and to handle them by a matrix equation we have to extend the matrix Eq. (E2). Then, the matrix coefficients, the solution variables, and the right hand sides become $i j$ dependent. For each $j$ we have an $A^{\prime \prime}(j)$ for all $i$. The next $A^{\prime \prime}(j+1)$ is placed at the rows below $A^{\prime \prime}(j)$ in the extended matrix. And the same 15 for the $v$ 's and the $R H S$ 's.

We create an extended matrix equation where we put the $A$ "'s at the position of the coefficients working on the central variables, which can be sketched like:

$$
\begin{aligned}
& \left(\begin{array}{llll}
A^{\prime \prime}(j=1) & & & \\
& A^{\prime \prime}(j=2) & & \\
& & \ddots & \\
& & A^{\prime \prime}\left(j=N_{\mathrm{y}}\right)
\end{array}\right)\left(\begin{array}{c}
v^{\prime \prime}(j=1) \\
v^{\prime \prime}(j=2) \\
\vdots \\
v^{\prime \prime}\left(j=N_{\mathrm{y}}\right)
\end{array}\right)=\left(\begin{array}{c}
R H S^{\prime \prime}(j=1) \\
R H S^{\prime \prime}(j=2) \\
\vdots \\
R H S^{\prime \prime}\left(j=N_{\mathrm{y}}\right)
\end{array}\right) \\
& \begin{array}{ccc}
\text { III } & \text { III, III } \\
A^{\prime} & V^{\prime} & R H S^{\prime}
\end{array}
\end{aligned}
$$

The rows in $A^{\prime}$ are longer than in the $A^{\prime \prime}$ 's because of the extra included $y$-neighbours. And if the $z$-derivatives have to be included implicitly, analogue to the previous step,

\section{GMDD}

2, 81-158, 2009

A numerical ice sheet - ice shelf 3D velocity field solution

T. J. Reerink et al.

Title Page

Abstract Introduction

Conclusions

Tables

References

Figures

14

$\rightarrow 1$

4

Back

Close

\section{Full Screen / Esc}

Printer-friendly Version

Interactive Discussion 
we have to extend the matrix Eq. (E3) by: placing $A^{\prime}(k+1)$ below $A^{\prime}(k)$ etc, like

$$
\begin{aligned}
& \left(\begin{array}{llll}
A^{\prime}(k=1) & & & \\
& A^{\prime}(k=2) & & \\
& & \ddots & \\
& & & A^{\prime}\left(k=N_{z}\right)
\end{array}\right)\left(\begin{array}{c}
v^{\prime}(k=1) \\
v^{\prime}(k=2) \\
\vdots \\
v^{\prime}\left(k=N_{z}\right)
\end{array}\right)=\left(\begin{array}{c}
R H S^{\prime}(k=1) \\
R H S^{\prime}(k=2) \\
\vdots \\
R H S^{\prime}\left(k=N_{z}\right)
\end{array}\right) \\
& \begin{array}{ccc}
\text { III } & \text { III } & \text { III } \\
A & \text { V } & R H S
\end{array}
\end{aligned}
$$

5 This is how the format of Eq. (E1) is organized.

Summarized in words: For a given $i j k$ combination a block of $n$ equations is placed in $A$, starting with the 1 st and ending with the $n$-th equation, where the solution vector $v$ is composed by placing $v_{1}(i j k) \ldots v_{n}(i j k)$ above each other. Having placed this $n$ equations for this $i j k$ combination, the next $n$ equations and variables for $i+1$ are 10 placed at the next $n$ rows in this matrix equation. And so on, up to $i=N_{\mathrm{x}}$. Then, all $i$ combinations for $j+1$ are placed, which is repeated up to $j=N_{\mathrm{y}}$. Below that, all $i j$ combinations for $k+1$ are placed, and so on for all the next $k$ up to $k=N_{z}$.

\section{E2 A more compact notation}

For a given $j k$ combination, the $n N_{\mathrm{x}}$ equations for all $i$ are given by Eq. (E2). This

Eq. (E2) can be represented more compact, first by writing only one block of $n$ equa-

GMDD

2, 81-158, 2009

A numerical ice sheet - ice shelf 3D velocity field solution

T. J. Reerink et al.

\section{Title Page}

Abstract

Introduction

Conclusions

References

Tables

Figures

14

$\rightarrow$

4

Back

Close 
tions for a certain $i$.

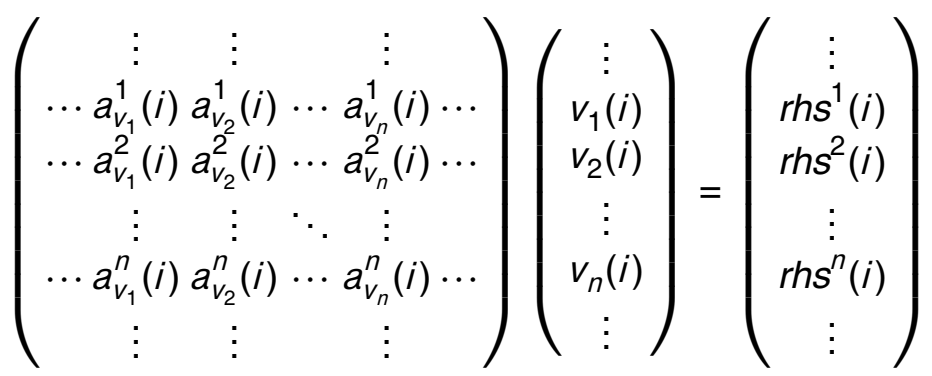

Here, $i$ represents $n$ equations, in that sense that only the central variables of that equations are shown in the solution vector while the neighbour variables are indicated by the dots up and below. In the matrix, the coefficients working on the central variables are shown while the coefficients working on the neighbour variables are indicated by the most left and most right horizontal dots. The matrix coefficients, the solution variables, and the RHS's for other $i$ are indicated by the dots up and below.

Secondly, this can be written more compact by introducing a counter / which equals the equation number. Actually, because / runs from 1 up to $n$, the $n$ successive equations are meant:

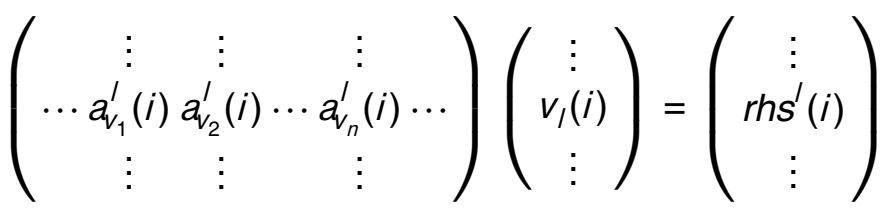

with / in the range:

$1 \leq l \leq n$

15 Finally, the $n$ matrix coefficients in Eq. (E6) working on the central variables be combined in a compact notation by introducing a counter $m$ which represents the $n$ suc-

A numerical ice sheet - ice shelf 3D velocity field solution

T. J. Reerink et al.

Title Page

Abstract

Introduction

Conclusions

Tables

References

Figures

14

4

Back

Close

\section{Full Screen / Esc}

Printer-friendly Version

Interactive Discussion 


$$
\left(\begin{array}{c}
\vdots \\
\cdots a_{v_{m}}^{\prime}(i) \cdots \\
\vdots
\end{array}\right)\left(\begin{array}{c}
\vdots \\
v_{l}(i) \\
\vdots
\end{array}\right)=\left(\begin{array}{c}
\vdots \\
r h s^{\prime}(i) \\
\vdots
\end{array}\right)
$$

with $m$ in the range:

$1 \leq m \leq n$

5 Equation (E8) is a very short notation for Eq. (E2) for which one has to realize that I and $m$ represent $n$ rows and columns respectively. The left and right dots in the matrix indicate the coefficients working on the neighbour variables. The other i's for this given $j k$ combination are indicated by the upper and lower dots in this matrix equation.

\section{E3 Relating the component names with their position}

10 The next step simultaneously handles two issues: Firstly, relating both the matrix coefficient names and the vector component names by a rule to their position in the matrix equation. Secondly, developing adequate names for the coefficients working on the neighbour variables, for which the earlier mentioned rule has to be valid as well. It is convenient for identification that the discretized variable names occur in the subscripts of the matrix coefficients. Because the coefficients in the matrix equal the factors in front of the discretized variables in the discretized equations, they easily can be placed in $A$ using the rule which relates the coefficient names with their positions.

A rule relating the name of a matrix coefficient with its position in a matrix, with a structure as described in Appendix E1, for a coefficient belonging to the /-th equation and working on an $m$-th variable $v_{m}$ (in this step only central variables are considered), is given by

$a_{\left(v_{m}\right)_{i, j}^{k}}^{\prime}\left[c_{1}, c_{m}\right]$

2, 81-158, 2009

A numerical ice sheet

- ice shelf 3D

velocity field solution

T. J. Reerink et al.

Title Page

Abstract

Introduction

Conclusions

Tables

References

Figures

14

I

4

Back

Close

Full Screen / Esc

Printer-friendly Version

Interactive Discussion 
with again / and $m$ in the range:

$1 \leq I \leq n$

$1 \leq m \leq n$

and with $\left(v_{m}\right)_{i, j}^{k}$ the $m$-th central variable. The $i j k$ indices of $v_{m}$ label the grid point

5 where the variable is defined on, and $\left(v_{m}\right)_{i, j}^{k}$ is a central variable because the indices just equal $i j k$. Closest neighbours would cary an additional -1 or +1 in those $i j k$ indices. Between brackets in rule $\mathrm{E} 10$, the row and column indices of the matrix coefficient are shown. Those row and column indices depend on ijk via the 'central index'. We call $c_{1}-c_{n}$ the first up to the $n$-th central index. Any $/$-th central index, $c_{l}$, is given 10 by

$c_{/}(i j k)=\operatorname{cindex}(i j k)+I$

for $1 \leq l \leq n$, with the central index, cindex, given by

cindex $(i j k)=n(i-1)+n(j-1) N_{\mathrm{x}}+n(k-1) N_{\mathrm{x}} N_{\mathrm{y}}$

for $1 \leq i \leq N_{\mathrm{x}}, 1 \leq j \leq N_{\mathrm{y}}$ and $1 \leq k \leq N_{z}$. The central indices are calculated once, for each block of $n$ equations for a certain $i j k$ combination.

The rule in $\mathrm{E} 10$ for coefficients working on the central variables can be generalized to a rule which is valid for all matrix coefficients by the following additions in the $i j k$ labels:

$a_{\left(v_{m}\right)_{i+d_{i}, j+d_{j}}^{k+d_{k}}}^{\prime}\left[c_{1}, c_{m}+d_{i} e_{i}+d_{j} e_{j}+d_{k} e_{k}\right]$

20 with $d_{i}, d_{j}$, and $d_{k}$ the distants (in grid units) in the $i, j$, and $k$ directions respectively between a neighbour grid point and the considered $i j k$ grid point. Where we have for

2, 81-158, 2009

A numerical ice sheet

- ice shelf 3D

velocity field solution

T. J. Reerink et al.

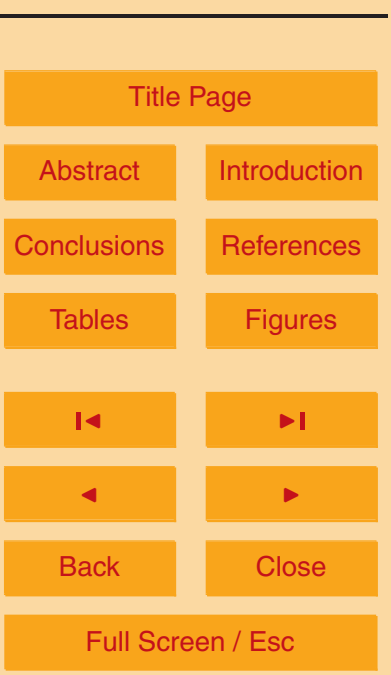

Printer-friendly Version

Interactive Discussion 
each $i j k$ combination

$$
\begin{aligned}
1-i & \leq d_{i} \leq N_{\mathrm{x}}-i \\
1-j & \leq d_{j} \leq N_{\mathrm{x}}-j \\
1-k & \leq d_{k} \leq N_{\mathrm{x}}-k
\end{aligned}
$$

5 Usually, as in our case, only $d_{i}, d_{j}$, and $d_{k}$ with values $-1,0$, or 1 (in case of high discretization accuracy: $-2,2)$ contribute, because they have matrix coefficients unequal to zero. The $e_{i}, e_{j}$, and $e_{k}$ in Eq. (E15) are the amounts of horizontally shifted places relative to the $m$-th row in respectively the $i, j$, and $k$ direction in matrix $A$ of two neighbour points situated side by side:

$10 e_{i}=n$

$e_{j}=n N_{\mathrm{x}}$

$e_{k}=n N_{\mathrm{x}} N_{\mathrm{y}}$

in accordance with the structure of matrix $A$ as desribed in Appendix $\mathrm{E} 1$.

For instance, a coefficient working on $U_{i+1, j-1}^{k}$ in the /-th equation looks like

$$
a_{\left(v_{1}\right)_{i+1, j-1}^{k}}^{l}\left[c_{1}, c_{1}+e_{i}-e_{j}\right]
$$

With the rule in E15 for the matrix coefficients and with an easier but analogue notation for the solution vector and the RHS vector, we construct a matrix equation in which the component names are related with their position in this matrix equation:

$$
\left(a_{\left(v_{m}\right)_{i+d_{i}, j+d_{j}}^{k+d_{k}}}^{\prime}(i j k)\left[c_{l}, c_{m}+d_{i} e_{i}+d_{j} e_{j}+d_{k} e_{k}\right]\right)\left(v_{l}(i j k)\left[c_{l}\right]\right)=\left(r h s^{\prime}(i j k)\left[c_{l}\right]\right)
$$

20 In Eq. (E23) the $i j k$ dependency of the central variables is omitted to keep the notation short. Equation (E23) relates any component name of the matrix equation with its position for all $i j k, l, m, d_{i}, d_{j}$, and $d_{k}$. Actually it describes the matrix equation in full component form in case one extends each variable for its entire range, in the same

GMDD

2, 81-158, 2009

A numerical ice sheet - ice shelf 3D velocity field solution

T. J. Reerink et al.

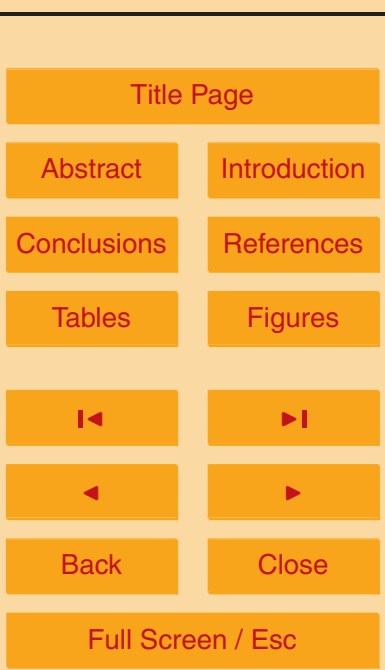

Printer-friendly Version

Interactive Discussion 
way as we did in Eqs. (E6-E9) for a given $j k$ combination. But Eq. (E23) is valid for any $i j k$ combination. All those $i j k, l, m, d_{i}, d_{j}$, and $d_{k}$ should be looped over their ranges to construct all components of the matrix equation. Therefore, the dots are not necessary anymore in this notation.

5 The relative column index is the additional part in the column index and denotes the relative distance to the coordinates $\left[c_{l}, c_{m}\right]$, i.e. in Eq. (E23) the relative indices are $d_{i} e_{i}+d_{j} e_{j}+d_{k} e_{k}$. The location for a given $i j k$ combination in $A$ of a coefficient $a_{v_{m}}^{\prime}$ working on an $m$-th central variable in the $/$-th equation, is $\left[c_{l}, c_{m}\right]$.

\section{E4 The compact three vector format}

10 Because $A$ is a huge $N x N$ but sparse matrix, our final interest is to give a compact 'three vector format' of $A$. In this three vector format only the non-zero coefficients of $A$ are stored. The first vector contains the values of the coefficients of $A$ while the second and the third contain respectively the corresponding row and column indices in $A$ of those coefficients. For this compact vector format of $A$ we have to know the indices of the matrix coefficients. We use the previous developed general notation for a matrix equation in which the names of the coefficients contain the name of the variable they work on. Then, the location of these matrix coefficients in $A$ are related to their names with rule (E15).

Equation (E23) concerns any component of the matrix equation, while we are interested in a describtion of a typical sparse matrix equation. Therefore, we develop a notation for the same matrix equation for which we realize it is sparse, which means a limited number of neighbours is involved per equation.

In a sparse case, most of the $d_{i}, d_{j}, d_{k}$-range combinations will not contribute. Even some $m$ cases of $v_{m}$ may not occur in some equations. Therefore, we have for each 25 /-th equation a small selection of $q$, coefficients in $A$ being non-zero. We label, for each I, those non-zero matrix coefficients by a subscript with a number between 1 and

GMDD

2, 81-158, 2009

A numerical ice sheet - ice shelf 3D velocity field solution

T. J. Reerink et al.

Title Page

Abstract Introduction

Conclusions

Tables

References

Figures

14

$\rightarrow 1$

4

Back

Close

Printer-friendly Version

Interactive Discussion 
$q_{1}$

$\left(a_{1}^{\prime}(i j k)\left[c_{1}, c_{m}+r_{1}^{\prime}\right] \ldots a_{q_{l}}^{\prime}(i j k)\left[c_{1}, c_{m}+r_{q_{l}}^{\prime}\right]\right)\left(v_{1}(i j k)\left[c_{1}\right]\right)=\left(r h s^{\prime}(i j k)\left[c_{1}\right]\right)$

with $r_{1}^{\prime} \ldots r_{q_{1}}^{\prime}$ the relative column indices, relative to $\left[c_{l}, c_{m}\right]$. The matrix coefficients carry in this sparse notation a number in their subscript instead of variable name. For 5 this limited number of non-zero coefficients, this coefficient names have to be specified. It depends on the shape of the equation, the implicit/explicit choice, and the discretization accuracy, which and how many terms are included. In our case, the non-zero matrix coefficients in the discretized equations are with help of the name-position rule (E15), listed in Tables 6-8 for the first, the second, and the third equation respectively.

10 Equation (E24) can be written more compact by using a sparse counter $s$, looping from 1 up to $q$, for a certain /

$$
\left(a_{s}^{\prime}(i j k)\left[c_{1}, c_{m}+r_{s}^{\prime}\right]\right)\left(v_{1}(i j k)\left[c_{1}\right]\right)=\left(r h s^{\prime}(i j k)\left[c_{1}\right]\right)
$$

where the combinations of $m, d_{i}, d_{j}, d_{k}$, which lead to the non-zero matrix coefficients, are stored with their row and column indices in the compact three vector format.

15 The exact non-zero coefficient names have to be read from the discretized equations. Those discretized variable names combined with rule (E15) give their positions in matrix $A$. For each / in Eq. (E25) we have $q$, matrix coefficients and $q$, relative column indices:

$a_{1}^{\prime} \leq a_{s}^{\prime} \leq a_{q_{1}}^{\prime}$

$20 \quad r_{1}^{\prime} \leq r_{s}^{\prime} \leq r_{q_{1}}^{\prime}$

with $I, q_{1}$, and $s$ in the range:

$1 \leq l \leq n$

$q_{1} \leq q_{1} \leq q_{n}$

2, 81-158, 2009

A numerical ice sheet

- ice shelf 3D

velocity field solution

T. J. Reerink et al.

$1 \leq s \leq q_{\text {, }}$ 
with a different amount of $q$, matrix coefficients for each of the / equations. Knowing their positions we can construct the $n$ arrays containing the relative column indices: $\left\{r_{1}^{1}, \ldots, r_{q_{1}}^{1}\right\},\left\{r_{1}^{2}, \ldots, r_{q_{2}}^{2}\right\}, \ldots,\left\{r_{1}^{n}, \ldots, r_{q_{n}}^{n}\right\}$.

Writing out Eq. (E25) for all / we have:

$$
\left(\begin{array}{ccc}
a_{1}^{1}\left[c_{1}, c_{m}+r_{1}^{1}\right] \ldots & a_{q_{1}}^{1}\left[c_{1}, c_{m}+r_{q_{1}}^{1}\right] \\
a_{1}^{2}\left[c_{2}, c_{m}+r_{1}^{2}\right] \ldots & a_{q_{2}}^{2}\left[c_{2}, c_{m}+r_{q_{2}}^{2}\right] \\
\vdots & \ddots & \vdots \\
a_{1}^{n}\left[c_{n},\right. & \left.c_{m}+r_{1}^{n}\right] \ldots & a_{q_{n}}^{n}\left[c_{n}, c_{m}+r_{q_{n}}^{n}\right]
\end{array}\right)\left(\begin{array}{c}
v_{1}\left[c_{1}\right] \\
v_{2}\left[c_{2}\right] \\
\vdots \\
v_{n}\left[c_{n}\right]
\end{array}\right)=\left(\begin{array}{c}
r h s^{1}\left[c_{1}\right] \\
r h s^{2}\left[c_{2}\right] \\
\vdots \\
r h s^{n}\left[c_{n}\right]
\end{array}\right)
$$

where the dependency of the components and of the central indices on $i j k$ is omitted, to keep the notation short.

For CPU reasons Eq. (E25) can be written, with use of $c_{m}=c_{l}-I+m$, as

$$
\left(a_{s}^{\prime}(i j k)\left[c_{1}, c_{l}+p_{s}^{\prime}\right]\right)\left(v_{1}(i j k)\left[c_{1}\right]\right)=\left(r h s^{\prime}(i j k)\left[c_{1}\right]\right)
$$

10 with $p_{s}^{\prime}=-I+m+r_{s}^{l}, p_{s}^{l}$ is relative to $\left[c_{l}, c_{l}\right]$.

This additional column indices $r_{s}^{\prime}$ are called the relative column indices for the /-th equation working on the $s$-th neighbour, because they give the the position relative to the $m$-th row at position $\left[c_{l}, c_{m}\right]$. We have for an /-th equation the relative column indices $r_{s}^{l}$ with $1 \leq s \leq q_{1}$, where $q$ is the number of non-zero matrix coefficients for that $15 l$. The numbers $q$, can differ for each of the $n$ physical equations depending on the nature of each equations, the way of discretization, and the implicit/explicit choices.

Together with Eqs. (E13-E14) Eq. (E25) represents the complete format for matrix $A$ describing $n$ coupled equations on a $3 \mathrm{D}$ grid.

All type of problems with $n$ equations and $n$ variables on a $3 \mathrm{D}$ grid can be handled with Eq. (E25), the problem specific information is stored in the short lists $a_{s}^{\prime}$ and $r_{s}^{\prime}$. Changing the previous choices concerning the shape of the equation, the implicit/explicit choice and the discretization accuracy, just needs adaption of those sets $a_{s}^{\prime}$ and $r_{s}^{\prime}$, but not in the rest of the story.

\section{GMDD}

2, 81-158, 2009

A numerical ice sheet - ice shelf 3D velocity field solution

T. J. Reerink et al.

Title Page

Abstract Introduction

Conclusions

Tables References Figures

14 $\rightarrow$

4

Back

Close

Full Screen / Esc

Printer-friendly Version

Interactive Discussion 
Programming this matrix equation exactly matches with looping the expression in Eq. (E25) over $s, I$ and $i j k$, after composing the $n$ arrays containing the non-zero matrix coefficients:

$\left\{a_{1}^{1}, \ldots, a_{q 1}^{1}\right\},\left\{a_{1}^{2}, \ldots, a_{q 2}^{2}\right\}, \ldots,\left\{a_{1}^{n}, \ldots, a_{q n}^{n}\right\}$

5 and the relative column indices:

$\left\{r_{1}^{1}, \ldots, r_{q 1}^{1}\right\},\left\{r_{1}^{2}, \ldots, r_{q 2}^{2}\right\}, \ldots,\left\{r_{1}^{n}, \ldots, r_{q n}^{n}\right\}$

If we follow this $s, I, i j k$ looping order in which only the non-zero matrix elements are counted, three vectors can be filled: one with the matrix coefficients, one with the corresponding row indices of that matrix coefficients and one with the corresponding column indices. We call this the compact vector format of the sparse matrix, because in this three vectors the complete content of this sparse matrix is stored ommiting the overwhelming majority of zero-components.

\section{E5 For a $d$ dimensional grid}

We showed the case of a 3D grid, it can be extended to a $d$ dimensional grid by taking the $d$ grid dimensions $i_{1} \ldots i_{d}$ instead of the three grid dimensions $i j k$. Further, in Eq. (E25) we have to replace $i j k$ by $i_{1} \ldots i_{d}$, and the cindex is generalized by

$\operatorname{cindex}\left(i_{1} \ldots i_{d}\right)=n \sum_{d^{\prime}=1}^{d}\left(\prod_{p=1}^{d^{\prime}}\left(i_{p}-1\right) N_{i_{(p-1)}}\right) \quad$ with $\quad N_{i_{0}} \equiv 1$

with $1 \leq i_{p} \leq N_{i_{p}}$ for the $p$-th grid direction. The dimension $N$ of $v$ and $R H S$ becomes

$N=n \prod_{p=1}^{d} N_{i_{p}}$

\section{GMDD}

2, 81-158, 2009

A numerical ice sheet - ice shelf 3D velocity field solution

T. J. Reerink et al.

Title Page

Abstract

Introduction

Conclusions

Tables

References

Figures

14

$\rightarrow 1$

4

Back

Close

Full Screen / Esc

Printer-friendly Version

Interactive Discussion 
The general equation format for $n$ equations with $n$ variables on a $d$ dimensional grid becomes

$$
\left.\left(a_{\left(v_{m}\right)}^{\prime}\right)_{i+d_{i}, j+d_{j}}^{k+d_{k}}\left(i_{1} \ldots i_{d}\right)\left[c_{l}, c_{m}+d_{i} e_{i}+d_{j} e_{j}+d_{k} e_{k}\right]\right)\left(v_{l}\left(i_{1} \ldots i_{d}\right)\left[c_{l}\right]\right)=\left(r h s^{\prime}\left(i_{1} \ldots i_{d}\right)\left[c_{l}\right]\right)
$$

5 where the dependency of the central variables on $i_{1} \ldots i_{d}$ is omitted to keep the notation short.

The sparse matrix format becomes

$$
\left(a_{s}^{\prime}\left(i_{1} \ldots i_{d}\right)\left[c_{1}, c_{m}+r_{s}^{\prime}\right]\right)\left(v_{1}\left(i_{1} \ldots i_{d}\right)\left[c_{1}\right]\right)=\left(r h s^{\prime}\left(i_{1} \ldots i_{d}\right)\left[c_{1}\right]\right)
$$

Acknowledgements. This work was initiated in the framework of the Utrecht Centre for Geosciences (UCG) programme.

\section{References}

Acheson, D.: Elementary Fluid Dynamics, Cambridge University Press, Oxford, 1st Edn., 2003. 89

Amestoy, P. R., Duff, I. S., and L'Excellent, J.-Y.: Multifrontal parallel distributed symmetric and unsymmetric solvers, Comput. Methods in Appl. Mech., 184, 501-520, 2000. 105

Balay, S., Buschelman, K., Eijkhout, V., Gropp, W. D., Kaushik, D., Knepley, M. G., McInnes, L. C., Smith, B. F., and Zhang, H.: PETSc Users Manual, Tech. Rep. ANL-95/11 - Revision 2.1.5, Argonne National Laboratory, 2004. 105

Deponti, A., Pennati, V., De Biase, L., Maggi, V., and Berta, F.: A new fully three-dimensional numerical model for ice dynamics, J. Glaciol., 52, 365-376, 2006. 94

Herterich, K.: On the flow within the transition zone between ice sheet and ice shelf, in: Dynamics of the West Antarctic ice sheet, edited by: Van der Veen, C. J. and Oerlemans, J., D. Reidel, Dordrecht, 185-202, 1987. 91

\section{GMDD}

2, 81-158, 2009

A numerical ice sheet - ice shelf 3D velocity field solution

T. J. Reerink et al.

Title Page

Abstract

Introduction

Conclusions

Tables

References

Figures

14

I

4

Back

Close

\section{Full Screen / Esc}

Printer-friendly Version

Interactive Discussion 
Hutter, K.: Theoretical Glaciology, material science of ice and the mechanics of glaciers and ice sheets, D. Reidel Publishing, Dordrecht, Terra Scientific Publishing, 1983. 83

Huybrechts, P.: The Antartic ice sheet and enviremental change: a three-dimensional modelling study, Ph.D. thesis, Alfred-Wegener-Institute for Polar and Marine Research, 1991. 83, 97

5 Mercer, J. H.: West Antarctic ice sheet and $\mathrm{CO}_{2}$ greenhouse effect: a threat of disaster, Nature, 271, 321-325, 1978. 83

Paterson, W.: The physics of glaciers, Pergamon, Copenhagen, 3rd Edn., 1994. 84, 92

Pattyn, F.: Ice-sheet modelling at different spatial resolutions: focus on the grounding zone, Ann. Glaciol., 31, 211-216, 2000. 91

10 Pattyn, F.: A new three-dimensional higher-order thermomechanical ice sheet model: Basic sensitivity, ice stream development, and ice flow across subglacial lakes, J. Geophys. Res., 108(B8), 2382, doi:10.1029/2002JB002329, 2003. 84, 91

Pattyn, F., Perichon, L., Aschwanden, A., Breuer, B., de Smedt, B., Gagliardini, O., Gudmundsson, G. H., Hindmarsh, R. C. A., Hubbard, A., Johnson, J. V., Kleiner, T., Konovalov, Y., Martin, C., Payne, A. J., Pollard, D., Price, S., Rückamp, M., Saito, F., Souček, O., Sugiyama, S., and Zwinger, T.: Benchmark experiments for higher-order and full-Stokes ice sheet models (ISMIPHOM), The Cryosphere, 2, 95-108, 2008. 84

Schoof, C.: Ice sheet grounding line dynamics: Steady states, stability, and hysteresis, J. Geophys. Res. (Earth Surface), 112, F03S28, doi:10.1029/2006JF000664, 2007. 83, 84

van Tuyll, C. I., van de Wal, R. S. W., and Oerlemans, J.: The response of a simple Antarctic ice-flow model to temperature and sea-level fluctuations over the Cenozoic era, Ann. Glaciol., 46, 69-77, 2007. 84

Vaughan, D. G.: West Antarctic Ice Sheet collapse the fall and rise of a paradigm, Climatic Change, 65-79, doi:10.1007/s10584-008-9448-3, 2007. 83

Vieli, A. and Payne, A.: Assessing the ability of numerical ice sheet models to simulate grounding line migration, J. Geophys. Res., 110, F01003, doi:10.1029/2004JF000202, 2005. 84

Zachos, J., Pagani, M., Sloan, S., Thomas, E., and Billups, K.: Trends, Rhythms, and Aberrations in Global Climate 65 Ma to Present, Science, 292, 686-693, 2001. 83

GMDD

2, 81-158, 2009

A numerical ice sheet - ice shelf 3D velocity field solution

T. J. Reerink et al.

Title Page

Abstract Introduction

Conclusions

Tables

References

Figures

14

$\rightarrow 1$

4

Back

Close

Printer-friendly Version

Interactive Discussion 
Table 1. Definitions of frequently used quantities and some math conventions.

\begin{tabular}{|c|c|c|c|}
\hline symbol & explanation & units & equation \\
\hline$x, y, z$ & spatial coordinates & $\mathrm{m}$ & \\
\hline$\zeta$ & $\begin{array}{l}\text { scaled vertical coordinate } \\
\zeta \equiv \frac{H_{S}-z}{H}\end{array}$ & - & (B8) \\
\hline$t$ & time coordinate & $\mathrm{s}$ & \\
\hline$H$ & ice thickness & $\mathrm{m}$ & (3.1), (3.2) \\
\hline$H_{s}$ & surface height & $\mathrm{m}$ & (3.1) \\
\hline$H_{b}$ & bedrock height & $\mathrm{m}$ & (3.1) \\
\hline$S$ & sea level & $\mathrm{m}$ & (3.1) \\
\hline$\rho_{\text {ice }}$ & density of ice & $\mathrm{kg} \mathrm{m}^{-3}$ & (3.1), (3.3) \\
\hline$\rho_{\mathrm{w}}$ & density of sea water & $\mathrm{kg} \mathrm{m}^{-3}$ & (3.1) \\
\hline$M_{s}$ & surface mass balance & $\mathrm{m}$ ice equivalent $\mathrm{y}^{-1}$ & (3.2) \\
\hline$M_{b}$ & basal melt & $\mathrm{m}$ ice equivalent $\mathrm{y}^{-1}$ & (3.2) \\
\hline$v_{\mathrm{i}}$ & i-th velocity component & $\mathrm{m} \mathrm{s}^{-1}$ & (3.3) \\
\hline $\bar{v}$ & vertical avarage velocity & $\mathrm{m} \mathrm{s}^{-1}$ & (3.2) \\
\hline$F_{\mathrm{i}}$ & summed volume force & $\mathrm{N}$ & (3.3), (3.6) \\
\hline$g$ & gravitational acceleration & $\mathrm{m} \mathrm{s}^{-2}$ & (3.6) \\
\hline$\tau_{\mathrm{ij}}$ & stress tensor & $\mathrm{N} \mathrm{m}^{-2}$ & (3.3), (3.7) \\
\hline$\tau_{\mathrm{ij}}^{?}$ & deviatoric stress tensor & $\mathrm{N} \mathrm{m}^{-2}$ & (3.19) \\
\hline$\dot{\epsilon}_{\mathrm{ij}}$ & stain rate & $s^{-1}$ & (3.18) \\
\hline$\dot{\epsilon}_{e}$ & minus the 2nd invariant of $\dot{\epsilon}$ & & $(3.20)$ \\
\hline$u$ & velocity in $x$-direction & $\mathrm{m} \mathrm{s}^{-1}$ & $(3.24),(4.1)$ \\
\hline$v$ & velocity in $y$-direction & $\mathrm{m} \mathrm{s}^{-1}$ & $(3.25),(4.2)$ \\
\hline$w$ & velocity in $z$-direction & $\mathrm{m} \mathrm{s}^{-1}$ & $(3.26),(4.3)$ \\
\hline$n$ & flow law exponent & - & (3.19) \\
\hline$A_{\text {flow }}$ & $\begin{array}{l}\text { ice flow parameter } \\
\text { ice temperature }\end{array}$ & $\mathrm{Pa}^{-n} \mathrm{~S}^{-1}$ & (3.19) \\
\hline$C^{\mathrm{uvw}}$ & abbreviation non linear part & & (3.23) \\
\hline$\partial_{\mathrm{i}}$ & $\frac{\partial}{\partial x_{i}}$ operator & & \\
\hline $\mathrm{d}_{t}$ & $\frac{d}{d t}$ operator & & \\
\hline$\delta_{\mathrm{ij}}$ & $\begin{array}{l}\delta_{\mathrm{ij}}=1 \text { for } i=j, \text { else } \delta_{\mathrm{ij}}=0 \\
\text { indicates a range }\end{array}$ & & \\
\hline
\end{tabular}

2, 81-158, 2009

A numerical ice sheet - ice shelf 3D velocity field solution

T. J. Reerink et al.

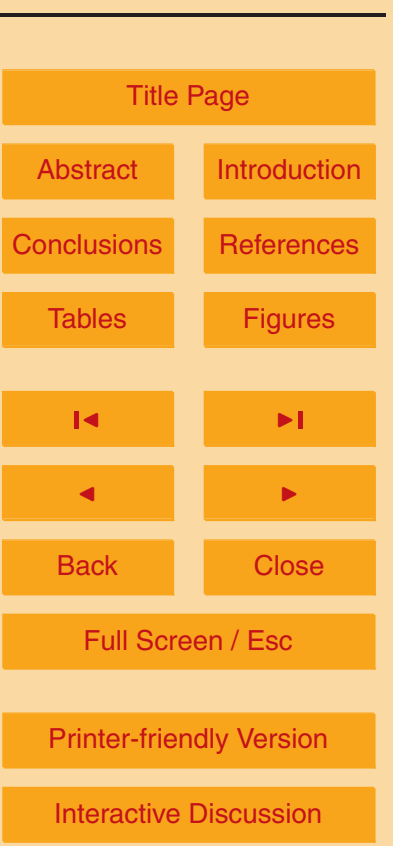




\section{GMDD}

2, 81-158, 2009

Table 2. The values of the simplification coefficients $f_{0}-f_{6}$ for the usual approximations at the sheet, the shelf, and within the transition zone (TZ). In case the simplification coefficients are adjusted to one, those momentum-equation terms listed are included in the calculation. If all $f_{0}-f_{6}$ equal one, the entire almost-full-stress solution is performed. Also the abbreviation $f_{56} \equiv 2 f_{5}+f_{6}$ is used further on.

\begin{tabular}{|c|c|c|c|c|c|}
\hline & sheet & $\mathrm{TZ}$ & shelf & involved terms & description \\
\hline$f_{0}$ & 0 & 0 & 0 & $\partial_{x} w, \partial_{y} w$ & 2nd part horizontal shear \\
\hline$f_{1}$ & 0 & 1 & 1 & $\tau_{\mathrm{xx}}^{\prime}, \tau_{\mathrm{yy}}^{\prime}$ & longitudinal stress \\
\hline$f_{2}$ & 0 & 1 & 1 & $\tau_{\mathrm{xy}}^{\prime}$ & side shear \\
\hline$f_{3}$ & 1 & 1 & 0 & $\tau_{\mathrm{xz}}^{\prime}, \tau_{\mathrm{yz}}^{\prime}$ & horizontal shear \\
\hline$f_{4}$ & 1 & 1 & 0 & $\begin{array}{l}\partial_{z} \tau_{\mathrm{xz}}^{\prime} \\
\partial_{z} \tau_{\mathrm{yz}}^{\prime}\end{array}$ & $\begin{array}{l}\text { vertical derivative } \\
\text { of horizontal shear }\end{array}$ \\
\hline$f_{5}$ & 0 & 0 & 1 & $\begin{array}{ll}\partial_{x} \tau_{\mathrm{xx}}^{\prime}, & \partial_{x} \tau_{\mathrm{yy}}^{\prime} \\
\partial_{y} \tau_{\mathrm{yy}}^{\prime}, & \partial_{y} \tau_{\mathrm{xx}}\end{array}$ & $\begin{array}{l}\text { horizontal derivative } \\
\text { of longitudinal stress }\end{array}$ \\
\hline$f_{6}$ & 0 & 0 & 1 & $\begin{array}{l}\partial_{y} \tau_{\mathrm{xy}} \\
\partial_{x} \tau_{\mathrm{xy}}^{\prime}\end{array}$ & $\begin{array}{l}\text { horizontal derivative } \\
\text { of side shear }\end{array}$ \\
\hline
\end{tabular}

A numerical ice sheet - ice shelf 3D velocity field solution

T. J. Reerink et al.

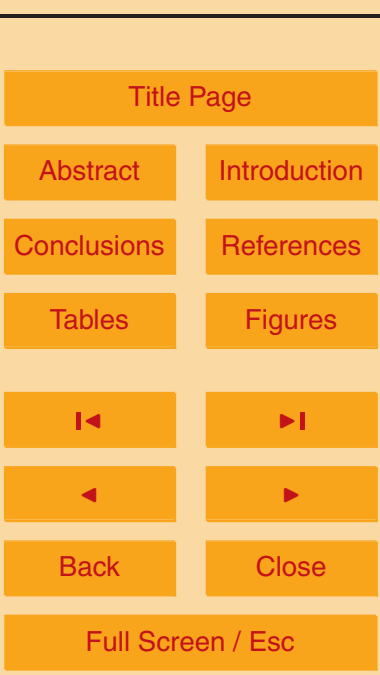

Printer-friendly Version

Interactive Discussion 
Table 3. The transformed derivative operators accompanying the coordinate transformation scaling the vertical ice coordinate.

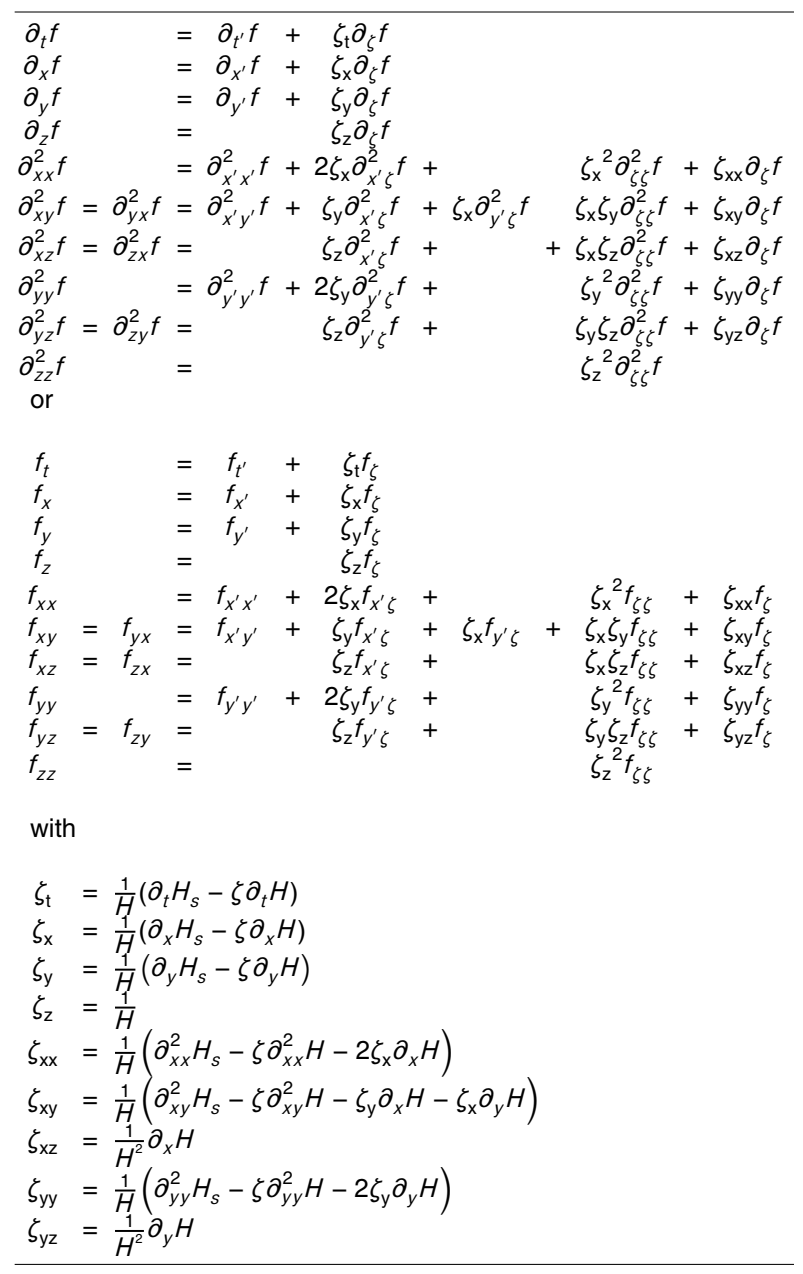

GMDD

2, 81-158, 2009

A numerical ice sheet - ice shelf 3D

velocity field solution

T. J. Reerink et al.

\section{Title Page}

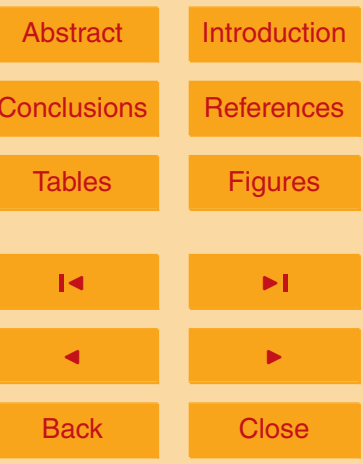

Full Screen / Esc

Printer-friendly Version

Interactive Discussion 
Table 4. The central finite difference discretizations (for a 3D field variable $f$ on an Arakawa A-grid).
GMDD

2, 81-158, 2009

A numerical ice sheet - ice shelf 3D velocity field solution

T. J. Reerink et al.

\section{Title Page}

with

$$
\begin{array}{ll}
a_{x}=-\frac{1}{2 \Delta x} & \mathrm{a}=\zeta_{\mathrm{k}}-\zeta_{\mathrm{k}-1} \\
c_{x}=\frac{1}{2 \Delta x} & \mathrm{~b}=\zeta_{\mathrm{k}+1}-\zeta_{\mathrm{k}} \\
a_{y}=-\frac{1}{2 \Delta y} & \\
c_{y}=\frac{1}{2 \Delta y} & \\
a_{\zeta}=-\frac{b}{a(a+b)} & \\
b_{\zeta}=\frac{b-a}{a b} & \\
c_{\zeta}=\frac{a}{b(a+b)} & \\
a_{x x}=\frac{1}{(\Delta x)^{2}} & \\
b_{x x}=-\frac{2}{(\Delta x)^{2}} & \\
c_{x x}=\frac{1}{(\Delta x)^{2}} & \\
a_{y y}=\frac{1}{(\Delta y)^{2}} & \\
b_{y y}=-\frac{2}{(\Delta y)^{2}} & \\
c_{y y}=\frac{1}{(\Delta y)^{2}} & \\
a_{\zeta \zeta}=\frac{2}{a(a+b)} & \\
b_{\zeta \zeta}=-\frac{2}{a b} & \\
c_{\zeta \zeta}=\frac{2}{b(a+b)} &
\end{array}
$$

Abstract

Introduction

Conclusions

References

Tables

Figures

14

$\rightarrow 1$

4

Back

Close

\section{Full Screen / Esc}

Printer-friendly Version

Interactive Discussion 
Table 5. The definitions of the coefficients in front of the derivatives of the scaled equations (D25-D24 or 5.6-5.5). The coefficients denoted by $f$ and $g$ concern the first and the second momentum equation respectively. The coefficients in front of the third equation need no extra definitions. The simplification coefficients $f_{0}-f_{6}$ and $f_{56}$ are as in Table 2.

\begin{tabular}{|c|c|c|c|}
\hline \multicolumn{2}{|r|}{ first equation } & \multicolumn{2}{|r|}{ second equation } \\
\hline 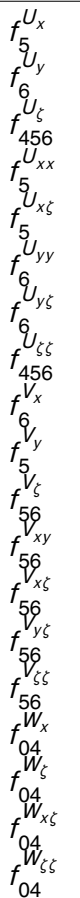 & $\begin{array}{l}=4 f_{5} C^{x} \\
=f_{6} C^{y} \\
=4 f_{5} C^{x} \zeta_{x}+f_{6} C^{y} \zeta_{y}+f_{4} C^{\zeta} \zeta_{z}+4 f_{5} \zeta_{x x}+f_{6} \zeta_{y y} \\
=4 f_{5} \\
=8 f_{5} \zeta_{x} \\
=f_{6} \\
=2 f_{6} \zeta_{y} \\
=4 f_{5} \zeta_{x}^{2}+f_{6} \zeta_{y}^{2}+f_{4} \zeta_{z}^{2} \\
=f_{6} C^{y} \\
=2 f_{5} C^{x} \\
=f_{6} C^{y} \zeta_{x}+2 f_{5} C^{x} \zeta_{y}+f_{56} \zeta_{x y} \\
=f_{56} \\
=f_{56} \zeta_{y} \\
=f_{56} \zeta_{x} \\
=f_{56} \zeta_{x} \zeta_{y} \\
=f_{0} f_{4} C^{\zeta} \\
=f_{0} f_{4} C^{\zeta} \zeta_{x}+f_{0} f_{4} \zeta_{x z} \\
=f_{0} f_{4} \zeta_{z} \\
=f_{0} f_{4} \zeta_{x} \zeta_{z}\end{array}$ & $\begin{array}{l}g_{5}^{U_{x}} \\
g_{6}^{U_{y}} \\
g_{56}^{U_{\zeta}} \\
g_{56}^{U_{x y}} \\
g_{56}^{U_{x \zeta}} \\
g_{56}^{U_{y \zeta}} \\
g_{56}^{V_{\zeta \zeta}} \\
g_{6}^{V_{x}} \\
g_{5}^{V_{y}} \\
g_{456}^{V_{\zeta}} \\
g_{6}^{V_{x x}} \\
g_{6}^{V_{x \zeta}} \\
g_{5 y}^{V_{y y}} \\
g_{5}^{V_{y \zeta}} \\
g_{456}^{V_{\zeta \zeta}} \\
g_{04}^{W_{y}} \\
g_{04}^{W_{\zeta}} \\
g_{04}^{W_{\zeta \zeta}} \\
g_{04}\end{array}$ & $\begin{array}{l}=2 f_{5} C^{y} \\
=f_{6} C^{x} \\
=2 f_{5} C^{y} \zeta_{x}+f_{6} C^{x} \zeta_{y}+f_{56} \zeta_{x y} \\
=f_{56} \\
=f_{56} \zeta_{y} \\
=f_{56} \zeta_{x} \\
=f_{56} \zeta_{x} \zeta_{y} \\
=f_{6} C^{x} \\
=4 f_{5} C^{y} \\
=f_{6} C^{x} \zeta_{x}+4 f_{5} C^{y} \zeta_{y}+f_{4} C^{\zeta} \zeta_{z}+f_{6} \zeta_{x x}+4 f_{5} \zeta_{y y} \\
=f_{6} \\
=2 f_{6} \zeta_{x} \\
=4 f_{5} \\
=8 f_{5} \zeta_{y} \\
=f_{6} \zeta_{x}^{2}+4 f_{5} \zeta_{y}^{2}+f_{4} \zeta_{z}^{2} \\
=f_{0} f_{4} C^{\zeta} \\
=f_{0} f_{4} C^{\zeta} \zeta_{y}+f_{0} f_{4} \zeta_{y z} \\
=f_{0} f_{4} \zeta_{z} \\
=f_{0} f_{4} \zeta_{y} \zeta_{z}\end{array}$ \\
\hline
\end{tabular}

GMDD

2, 81-158, 2009

A numerical ice sheet - ice shelf 3D velocity field solution

T. J. Reerink et al.

\section{Title Page}

Abstract

Introduction

Conclusions

References

Tables

Figures

14

$\rightarrow$

4

Back

Close

\section{Full Screen / Esc}

Printer-friendly Version

Interactive Discussion 
Table 6. The 43 matrix coefficients $a_{s}^{\prime}(i j k)\left[c_{,}, c_{m}+r_{s}^{\prime}\right]$ for the first equation: $l=1,1 \leq s \leq 43$, which are the coefficients in front of the discretized variables in Eq. (D30). Between brackets the row and column indices of the location of each coefficient in the matrix, which is part of Eq. (5.21), are shown. The $i j k$ dependence of the coefficients and the central indices are omitted to keep the notation short. Far left shows the variable of the solution vector where the coefficient works on. The next table column shows the coefficient name in "sparse" notation: The subscript equals the sparse index $1 \leq s \leq q_{1}$. The row index equals the central index $c_{l}$, while the column index is equal to the central index $c_{m}$ plus the relative column index $r_{s}^{l}$. The third table column contains real names of the coefficients and the indices. Far right, the values of the coefficients are defined. Note that the three vertical parts of the table concern the cases $m=1$ up to $m=3$ top-down respectively. The coefficients denoted by $f$ and $g$ are defined in Table 5 . The precise definitions of the indices can be found in Table 8 .

$$
\begin{aligned}
& U_{i-1, j}^{k}: a^{1}{ }_{1}\left[c_{1}, c_{1}+r^{1}{ }_{1}\right]=a_{\left(v_{1}\right)_{i-1, j}^{k}}^{1}\left[c_{1}, c_{1}-e_{i}\right]=a_{x} f_{5}^{U_{x}}+a_{x x} f_{5}^{U_{x x}}+a_{x} b_{\zeta} f_{5}^{U_{x \zeta}} \\
& U_{i+1, j}^{k}: a^{1}{ }_{2}\left[c_{1}, c_{1}+r^{1}{ }_{2}\right]=a_{\left(v_{1}\right)_{i+1, j}^{k}}^{1}\left[c_{1}, c_{1}+e_{i}\right]=c_{x} f_{5}^{U_{x}}+c_{x x} f_{5}^{U_{x x}}+b_{\zeta} c_{x} f_{5} U_{x \zeta} \\
& U_{i, j-1}^{k}: a^{1}{ }_{3}\left[c_{1}, c_{1}+r^{1}{ }_{3}\right]=a_{\left(v_{1}\right)_{i, j-1}^{k}}^{1}\left[c_{1}, c_{1}-e_{j}\right]=a_{y} f_{6}^{U_{y}}+a_{y y} f_{6}^{U_{y y}}+a_{y} b_{\zeta} f_{6}^{U_{y \zeta}} \\
& U_{\mathrm{i}, \mathrm{j}+1}^{\mathrm{k}}: a^{1}{ }_{4}\left[c_{1}, c_{1}+r^{1}{ }_{4}\right]=a_{\left(v_{1}\right)_{i, j+1}^{k}}^{1}\left[c_{1}, c_{1}+e_{j}\right] \\
& U_{\mathrm{i}, \mathrm{j}}^{\mathrm{k}-1}: a^{1}{ }_{5}\left[c_{1}, c_{1}+r^{1}{ }_{5}\right]=a_{\left(v_{1}\right)_{i, j}^{k-1}}^{1}\left[c_{1}, c_{1}-e_{k}\right] \\
& U_{\mathrm{i}, \mathrm{j}}^{\mathrm{k}}: a_{6}^{1}\left[c_{1}, c_{1}+r_{6}^{1}{ }_{6}\right]=a_{\left(\mathrm{v}_{1}\right)_{i, j}^{k}}^{1}\left[c_{1}, c_{1}\right] \\
& U_{\mathrm{i}, \mathrm{j}}^{\mathrm{k}+1}: a^{1}{ }_{7}\left[c_{1}, c_{1}+r^{1}{ }_{7}\right]=a_{\left(v_{1}\right)_{i, j}^{k+1}}^{1}\left[c_{1}, c_{1}+e_{k}\right] \\
& =c_{y} f_{6}^{U_{y}}+c_{y y} f_{6}^{U_{y y}}+b_{\zeta} c_{y} f_{6}^{U_{y \zeta}} \\
& =a_{\zeta} f_{456}^{U_{\zeta}}+a_{\zeta \zeta} f_{456}^{U_{\zeta \zeta}} \\
& =b_{\zeta} f_{456}^{U_{\zeta}}+b_{x x} f_{5}^{U_{x x}}+b_{y y} f_{6}^{U_{y y}}+b_{\zeta \zeta} f_{456}^{U_{\zeta \zeta}} \\
& =c_{\zeta} f_{456}^{U_{\zeta}}+c_{\zeta \zeta} f_{456}^{U_{\zeta \zeta}} \\
& U_{i-1, j}^{k-1}: a^{1}{ }_{8}\left[c_{1}, c_{1}+r^{1}{ }_{8}\right]=a_{\left(V_{1}\right)_{i-1, j}^{k-1}}^{1}\left[c_{1}, c_{1}-e_{i}-e_{k}\right]=a_{x} a_{\zeta} f_{5} U_{x \zeta} \\
& U_{i+1, j}^{k-1}: a^{1}{ }_{9}\left[c_{1}, c_{1}+r^{1}{ }_{9}\right]=a_{\left(v_{1}\right)_{i+1, j}^{k-1}}^{1}\left[c_{1}, c_{1}+e_{i}-e_{k}\right]=a_{\zeta} c_{x} f_{5} U_{x \zeta} \\
& U_{\mathrm{i}-1, \mathrm{j}}^{\mathrm{k}+1}: a_{10}^{1}\left[c_{1}, c_{1}+r_{10}^{1}\right]=a_{\left(v_{1}\right)_{i-1, j}^{k+1}}^{1}\left[c_{1}, c_{1}-e_{i}+e_{k}\right]=a_{x} c_{\zeta} f_{5} U_{x \zeta} \\
& U_{\mathrm{i}+1, j}^{k+1}: a_{11}^{1}\left[c_{1}, c_{1}+r_{11}^{1}\right]=a_{\left(v_{1}\right)_{i+1, j}^{k+1}}^{1}\left[c_{1}, c_{1}+e_{i}+e_{k}\right]=c_{x} c_{\zeta} f_{5}^{U_{x \zeta}} \\
& U_{i, j-1}^{k-1}: a_{12}^{1}\left[c_{1}, c_{1}+r_{12}^{1}\right]=a_{\left(v_{1}\right)_{i, j-1}^{k-1}}^{1}\left[c_{1}, c_{1}-e_{j}-e_{k}\right]=a_{y} a_{\zeta} f_{6} U_{y \zeta} \\
& U_{i, j+1}^{k-1}: a_{13}^{1}\left[c_{1}, c_{1}+r_{13}^{1}\right]=a_{\left(v_{1}\right)_{i, j+1}^{k-1}}^{1}\left[c_{1}, c_{1}+e_{j}-e_{k}\right]=a_{\zeta} c_{y} f_{6} U_{y \zeta} \\
& U_{i, j-1}^{k+1}: a_{14}^{1}\left[c_{1}, c_{1}+r_{14}^{1}\right]=a_{\left(v_{1}\right)_{i, j-1}^{k+1}}^{1}\left[c_{1}, c_{1}-e_{j}+e_{k}\right]=a_{y} c_{\zeta} f_{6}^{U_{y \zeta}} \\
& U_{i, j+1}^{k+1}: a_{15}^{1}\left[c_{1}, c_{1}+r_{15}^{1}\right]=a_{\left(v_{1}\right)_{i, j+1}^{k+1}}^{1}\left[c_{1}, c_{1}+e_{j}+e_{k}\right]=c_{y} c_{\zeta} f_{6}^{U_{y \zeta}}
\end{aligned}
$$

2, 81-158, 2009

\section{A numerical ice sheet - ice shelf 3D \\ velocity field solution}

T. J. Reerink et al.

\section{Title Page}

Abstract

Introduction

Conclusions

References

Tables

Figures

14

$\rightarrow$

4

Back

Close

\section{Full Screen / Esc}

Printer-friendly Version

Interactive Discussion 


\section{GMDD}

Table 6. Continued.

2, 81-158, 2009

$$
\begin{aligned}
& V_{\mathrm{i}-1, \mathrm{j}}^{\mathrm{k}}: a_{16}^{1}\left[c_{1}, c_{2}+r_{16}^{1}\right]=a_{\left(v_{2}\right)_{i-1}^{k}}^{1}\left[c_{1}, c_{2}-e_{i}\right]=a_{x} f_{6}^{V_{X}}+a_{x} b_{\zeta} f_{56}^{V_{x \zeta}} \\
& V_{i+1, j}^{k}: a_{17}^{1}\left[c_{1}, c_{2}+r_{17}^{1}\right]=a_{\left(v_{2}\right)_{i+1, j}^{k}}^{1}\left[c_{1}, c_{2}+e_{i}\right]=c_{x} f_{6}^{V_{X}}+b_{\zeta} c_{x} f_{56}^{V_{X \zeta}} \\
& V_{i, j-1}^{k}: a_{18}^{1}\left[c_{1}, c_{2}+r_{18}^{1}\right]=a_{\left(v_{2}\right)_{i, j-1}^{k}}^{1}\left[c_{1}, c_{2}-e_{j}\right]=a_{y} f_{5}^{V_{y}}+a_{y} b_{\zeta} f_{56}^{V_{y \zeta}} \\
& V_{i, j+1}^{k}: a_{19}^{1}\left[c_{1}, c_{2}+r_{19}^{1}\right]=a_{\left(v_{2}\right)_{i, j+1}^{k}}^{1}\left[c_{1}, c_{2}+e_{j}\right]=c_{y} f_{5}^{V_{y}}+b_{\zeta} c_{y} f_{56}^{V_{y \zeta}} \\
& V_{i, j}^{k-1}: a_{20}^{1}\left[c_{1}, c_{2}+r_{20}^{1}\right]=a_{\left(v_{2}\right)_{i, j}^{k-1}}^{1}\left[c_{1}, c_{2}-e_{k}\right]=a_{\zeta} f_{56} V_{\zeta}+a_{\zeta \zeta} f_{56} V_{\zeta \zeta}^{56} \\
& V_{i, j}^{k}: a_{21}^{1}\left[c_{1}, c_{2}+r_{21}^{1}\right]=a_{\left(v_{2}\right)_{i, j}^{k}}^{1}\left[c_{1}, c_{2}\right] \quad=b_{\zeta} f_{56}^{V_{\zeta}}+b_{\zeta \zeta} f_{56} v_{\zeta \zeta} \\
& V_{i, j}^{k+1}: a_{22}^{1}\left[c_{1}, c_{2}+r_{22}^{1}\right]=a_{\left(v_{2}\right)_{i, j}^{k+1}}^{1}\left[c_{1}, c_{2}+e_{k}\right]=c_{\zeta} f_{56}^{V_{\zeta}}+c_{\zeta \zeta} f_{56}^{V_{\zeta \zeta}} \\
& V_{i-1, j}^{k-1}: a_{23}^{1}\left[c_{1}, c_{2}+r_{23}^{1}\right]=a_{\left(v_{2}\right)_{i-1, j}^{k-1}}^{1}\left[c_{1}, c_{2}-e_{i}-e_{k}\right]=a_{x} a_{\zeta} f_{56}^{V_{x \zeta}} \\
& V_{i+1, j}^{k-1}: a_{24}^{1}\left[c_{1}, c_{2}+r_{24}^{1}\right]=a_{\left(v_{2}\right)_{i+1, j}^{k-1}}^{1}\left[c_{1}, c_{2}+e_{i}-e_{k}\right]=a_{\zeta} c_{x} f_{56} V_{X \zeta} \\
& V_{i-1, j}^{k+1}: a_{25}^{1}\left[c_{1}, c_{2}+r_{25}^{1}\right]=a_{\left(v_{2}\right)_{i-1, j}^{k+1}}^{1}\left[c_{1}, c_{2}-e_{i}+e_{k}\right]=a_{x} c_{\zeta} f_{56}^{V_{x \zeta}} \\
& V_{i+1, j}^{k+1}: a_{26}^{1}\left[c_{1}, c_{2}+r_{26}^{1}\right]=a_{\left(v_{2}\right)_{i+1, j}^{k+1}}^{1}\left[c_{1}, c_{2}+e_{i}+e_{k}\right]=c_{x} c_{\zeta} f_{56}^{V_{x \zeta}} \\
& V_{\mathrm{i}, j-1}^{k-1}: a_{27}^{1}\left[c_{1}, c_{2}+r_{27}^{1}\right]=a_{\left(v_{2}\right)_{i, j-1}^{k-1}}^{1}\left[c_{1}, c_{2}-e_{j}-e_{k}\right]=a_{y} a_{\zeta} f_{56} V_{y \zeta} \\
& V_{\mathrm{i}, j+1}^{k-1}: a_{28}^{1}\left[c_{1}, c_{2}+r_{28}^{1}\right]=a_{\left(v_{2}\right)_{j, j+1}^{k-1}}^{1}\left[c_{1}, c_{2}+e_{j}-e_{k}\right]=a_{\zeta} c_{y} f_{56}^{V_{y \zeta}} \\
& V_{i, j-1}^{k+1}: a_{29}^{1}\left[c_{1}, c_{2}+r_{29}^{1}\right]=a_{\left(v_{2}\right)_{i, j-1}^{k+1}}^{1}\left[c_{1}, c_{2}-e_{j}+e_{k}\right]=a_{y} c_{\zeta} f_{56}^{V_{y \zeta}} \\
& V_{\mathrm{i}, j+1}^{\mathrm{k}+1}: a_{30}^{1}\left[c_{1}, c_{2}+r_{30}^{1}\right]=a_{\left(v_{2}\right)_{i, j+1}^{k+1}}^{1}\left[c_{1}, c_{2}+e_{j}+e_{k}\right]=c_{y} c_{\zeta} f_{56}^{V_{y \zeta}} \\
& V_{i-1, j-1}^{k}: a_{31}^{1}\left[c_{1}, c_{2}+r_{31}^{1}\right]=a_{\left(v_{2}\right)_{i-1, j-1}^{k}}^{1}\left[c_{1}, c_{2}-e_{i}-e_{j}\right]=a_{x} a_{y} f_{56}^{V_{x y}} \\
& V_{\mathrm{i}-1, j+1}^{\mathrm{k}}: a_{32}^{1}\left[c_{1}, c_{2}+r_{32}^{1}\right]=a_{\left(v_{2}\right)_{i-1, j+1}^{k}}^{1}\left[c_{1}, c_{2}-e_{i}+e_{j}\right]=a_{x} c_{y} f_{56}^{V_{x y}} \\
& V_{i+1, j-1}^{k}: a_{33}^{1}\left[c_{1}, c_{2}+r_{33}^{1}\right]=a_{\left(v_{2}\right)_{i+1, j-1}^{k}}^{1}\left[c_{1}, c_{2}+e_{i}-e_{j}\right]=a_{y} c_{x} f_{56}^{V_{x y}} \\
& V_{i+1, j+1}^{\mathrm{k}}: a_{34}^{1}\left[c_{1}, c_{2}+r_{34}^{1}\right]=a_{\left(v_{2}\right)_{i+1, j+1}^{k}}^{1}\left[c_{1}, c_{2}+e_{i}+e_{j}\right]=c_{x} c_{y} f_{56}^{V_{x y}}
\end{aligned}
$$

A numerical ice sheet - ice shelf 3D velocity field solution

T. J. Reerink et al.

\section{Title Page}

\section{Full Screen / Esc}

Printer-friendly Version 


\section{GMDD}

2, 81-158, 2009

A numerical ice sheet

- ice shelf 3D

velocity field solution

Table 6. Continued.

$$
\begin{aligned}
& W_{i-1, j}^{k}: a_{35}^{1}\left[c_{1}, c_{3}+r_{35}^{1}\right]=a_{\left(v_{3}\right)_{i-1, j}^{k}}^{1}\left[c_{1}, c_{3}-e_{i}\right]=a_{x} f_{04}^{W_{x}}+a_{x} b_{\zeta} f_{04}^{W_{x \zeta}} \\
& W_{\mathrm{i}+1, j}^{\mathrm{k}}: a_{36}^{1}\left[c_{1}, c_{3}+r_{36}^{1}\right]=a_{\left(v_{3}\right)_{i+1, j}^{k}}^{1}\left[c_{1}, c_{3}+e_{i}\right]=c_{x} f_{04} W_{x}+b_{\zeta} c_{x} f_{04}^{W_{x \zeta}} \\
& W_{i, j}^{k-1}: a_{37}^{1}\left[c_{1}, c_{3}+r_{37}^{1}\right]=a_{\left(v_{3}\right)_{i, j}^{k-1}}^{1}\left[c_{1}, c_{3}-e_{k}\right]=a_{\zeta} f_{04} W_{\zeta}+a_{\zeta \zeta} f_{04} W_{\zeta \zeta} \\
& W_{i, j}^{k}: a_{38}^{1}\left[c_{1}, c_{3}+r_{38}^{1}\right]=a_{\left(v_{3}\right)_{i, j}^{k}}^{1}\left[c_{1}, c_{3}\right]=b_{\zeta} f_{04}^{w_{\zeta}}+b_{\zeta \zeta} w_{04}^{W_{\zeta \zeta}} \\
& W_{i, j}^{k+1}: a_{39}^{1}\left[c_{1}, c_{3}+r_{39}^{1}\right]=a_{\left(v_{3}\right)_{i, j}^{k+1}}^{1}\left[c_{1}, c_{3}+e_{k}\right]=c_{\zeta} f_{04}^{W_{\zeta}}+c_{\zeta \zeta} f_{04}^{W_{\zeta \zeta}} \\
& W_{\mathrm{i}-1, j}^{\mathrm{k}-1}: a_{40}^{1}\left[c_{1}, c_{3}+r_{40}^{1}\right]=a_{\left(v_{3}\right)_{i-1, j}^{k-1}}^{1}\left[c_{1}, c_{3}-e_{i}-e_{k}\right]=a_{x} a_{\zeta} f_{04}^{W_{x \zeta}} \\
& W_{\mathrm{i}+1, j}^{k-1}: a_{41}^{1}\left[c_{1}, c_{3}+r_{41}^{1}\right]=a_{\left(v_{3}\right)_{i+1, j}^{k-1}}^{1}\left[c_{1}, c_{3}+e_{i}-e_{k}\right]=a_{\zeta} c_{x} f_{04}^{W_{X \zeta}} \\
& W_{\mathrm{i}-1, j}^{\mathrm{k}+1}: a_{42}^{1}\left[c_{1}, c_{3}+r_{42}^{1}\right]=a_{\left(v_{3}\right)_{i-1, j}^{k+1}}^{1}\left[c_{1}, c_{3}-e_{i}+e_{k}\right]=a_{x} c_{\zeta} f_{04}^{W_{X \zeta}} \\
& W_{i+1, j}^{k+1}: a_{43}^{1}\left[c_{1}, c_{3}+r_{43}^{1}\right]=a_{\left(v_{3}\right)_{i+1, j}^{k+1}}^{1}\left[c_{1}, c_{3}+e_{i}+e_{k}\right]=c_{x} c_{\zeta} f_{04}^{W_{x \zeta}}
\end{aligned}
$$

T. J. Reerink et al.

\section{Title Page}

\section{Abstract}

Introduction

Conclusions

References

Tables

Figures

14

$>$ I

4

Back

\section{Full Screen / Esc}

Printer-friendly Version

Interactive Discussion 
Table 7. The 43 matrix coefficients $a_{s}^{\prime}(i j k)\left[c, c_{m}+r_{s}^{l}\right]$ for the second equation: $l=2,1 \leq s \leq 43$, which are the coefficients in front of the discretized variables in Eq. (D31). See for more explanation the caption of Table 6, and Table 8 for the precise definitions of the indices.
GMDD

2, 81-158, 2009

A numerical ice sheet - ice shelf 3D velocity field solution

T. J. Reerink et al.

Title Page

Abstract

Introduction

Conclusions

Tables

References

$U_{\mathrm{i}+1, \mathrm{j}}^{\mathrm{k}-1}: a^{2}{ }_{9}\left[c_{2}, c_{1}+r_{9}^{2}\right]=a_{\left(v_{1}\right)_{i+1, j}^{k-1}}^{2}\left[c_{2}, c_{1}+e_{i}-e_{k}\right]=a_{\zeta} c_{x} g_{56}^{U_{x \zeta}}$

$U_{i-1, j}^{k+1}: a_{10}^{2}\left[c_{2}, c_{1}+r_{10}^{2}\right]=a_{\left(v_{1}\right)_{j-1, j}^{k+1}}^{2}\left[c_{2}, c_{1}-e_{i}+e_{k}\right]=a_{x} c_{\zeta} g_{56}^{U_{x \zeta}}$

$U_{i+1, j}^{k+1}: a_{11}^{2}\left[c_{2}, c_{1}+r_{11}^{2}\right]=a_{\left(v_{1}\right)_{i+1, j}^{k+1}}^{2}\left[c_{2}, c_{1}+e_{i}+e_{k}\right]=c_{x} c_{\zeta} g_{56}^{U_{x \zeta}}$

$U_{\mathrm{i}, \mathrm{j}-1}^{k-1}: a_{12}^{2}\left[c_{2}, c_{1}+r_{12}^{2}\right]=a_{\left(v_{1}\right)_{i, j-1}^{k-1}}^{2}\left[c_{2}, c_{1}-e_{j}-e_{k}\right]=a_{y} a_{\zeta} g_{56}^{U_{y \zeta}}$

$U_{i, j+1}^{k-1}: a_{13}^{2}\left[c_{2}, c_{1}+r_{13}^{2}\right]=a_{\left(v_{1}\right)_{i, j+1}^{k-1}}^{2}\left[c_{2}, c_{1}+e_{j}-e_{k}\right]=a_{\zeta} c_{y} g_{56}^{U_{y \zeta}}$

$U_{i, j-1}^{k+1}: a_{14}^{2}\left[c_{2}, c_{1}+r_{14}^{2}\right]=a_{\left(v_{1}\right)_{i, j-1}^{k+1}}^{2}\left[c_{2}, c_{1}-e_{j}+e_{k}\right]=a_{y} c_{\zeta} g_{56}^{U_{y \zeta}}$

$U_{i, j+1}^{k+1}: a_{15}^{2}\left[c_{2}, c_{1}+r_{15}^{2}\right]=a_{\left(v_{1}\right)_{i j+1}^{k+1}}^{2}\left[c_{2}, c_{1}+e_{j}+e_{k}\right]=c_{y} c_{\zeta} g_{56}^{U_{y \zeta}}$

$U_{i-1, j-i}^{k} a_{16}^{2}\left[c_{2}, c_{1}+r_{16}^{2}\right]=a_{\left(v_{1}\right)_{i-1, j-1}^{k}}^{2}\left[c_{2}, c_{1}-e_{i}-e_{j} k a_{x} a_{y} g_{56}^{U_{x y}}\right.$

$U_{i-1, j+1}^{k} a_{17}^{2}\left[c_{2}, c_{1}+r_{17}^{2}\right]=a_{\left.\left(v_{1}\right)\right)_{i-1, j+1}^{k}}^{2}\left[c_{2}, c_{1}-e_{i}+e_{j} k a_{x} c_{y} g_{56}^{U_{x y}}\right.$

$U_{i+1, j-1}^{k}: a_{18}^{2}\left[c_{2}, c_{1}+r_{18}^{2}\right]=a_{\left(v_{1}\right)_{i+1, j-1}^{k}}^{2}\left[c_{2}, c_{1}+e_{i}-e_{j}=a_{y} c_{x} g_{56}^{U_{x y}}\right.$

14

Figures

$U_{i+1, j+1}^{k} ; a_{19}^{2}\left[c_{2}, c_{1}+r_{19}^{2}\right]=a_{\left(v_{1}\right)_{i+1, j+1}^{k}}^{2}\left[c_{2}, c_{1}+e_{i}+e_{j}=c_{x} c_{y} g_{56}^{U_{x y}}\right.$ 
Table 7. Continued.

$$
\begin{aligned}
& V_{i-1, j}^{k} a_{20}^{2}\left[c_{2}, c_{2}+r_{20}^{2}\right]=a_{\left(v_{2}\right)_{i-1, j}^{k}}^{2}\left[c_{2}, c_{2}-e_{i}\right]=a_{x} g_{6}^{V_{x}}+a_{x x} g_{6}^{V_{x x}}+a_{x} b_{\zeta} g_{6}^{V_{x \zeta}} \\
& V_{i+1, j}^{k} a_{21}^{2}\left[c_{2}, c_{2}+r_{21}^{2}\right]=a_{\left(v_{2}\right)_{i+1, j}^{k}}^{2}\left[c_{2}, c_{2}+e_{i}\right]=c_{x} g_{6}^{V_{x}}+c_{x x} g_{6}^{V_{x x}}+b_{\zeta} c_{x} g_{6}^{V_{x \zeta}} \\
& V_{i, j-1}^{k}: a_{22}^{2}\left[c_{2}, c_{2}+r_{22}^{2}\right]=a_{\left(v_{2}\right)_{i, j-1}^{k}}^{2}\left[c_{2}, c_{2}-e_{j}\right]=a_{y} g_{5}^{V_{y}}+a_{y y} g_{5}^{V_{y y}}+a_{y} b_{\zeta} g_{5}^{V_{y \zeta}} \\
& V_{i, j+1}^{k}: a_{23}^{2}\left[c_{2}, c_{2}+r_{23}^{2}\right]=a_{\left(v_{2}\right)_{i, j+1}^{k}}^{2}\left[c_{2}, c_{2}+e_{j}\right]=c_{y} g_{5}^{V_{y}}+c_{y y} g_{5}^{V_{y y}}+b_{\zeta} c_{y} g_{5}^{V_{y \zeta}} \\
& V_{i, j}^{k-1}: a_{24}^{2}\left[c_{2}, c_{2}+r_{24}^{2}\right]=a_{\left(v_{2}\right)_{i j}^{k-1}}^{2}\left[c_{2}, c_{2}-e_{k}\right] \quad=a_{\zeta} g_{456}^{V_{\zeta}}+a_{\zeta \zeta} g_{456}^{V_{\zeta \zeta}} \\
& V_{i, j}^{k}: a_{25}^{2}\left[c_{2}, c_{2}+r_{25}^{2}\right]=a_{\left(v_{2}\right)_{i, j}^{k}}^{2}\left[c_{2}, c_{2}\right] \\
& V_{\mathrm{i}, \mathrm{j}}^{\mathrm{k}+1}: a_{26}^{2}\left[c_{2}, c_{2}+r_{26}^{2}\right]=a_{\left(v_{2}\right)_{i, j}^{k+1}}^{2}\left[c_{2}, c_{2}+e_{k}\right] \\
& =b_{\zeta} g_{456}^{V_{\zeta}}+b_{x x} g_{6}^{V_{x x}}+b_{y y} g_{5}^{V_{y y}}+b_{\zeta \zeta} g_{456}^{v_{\zeta \zeta}} \\
& V_{i-1, j}^{k-1} a_{27}^{2}\left[c_{2}, c_{2}+r_{27}^{2}\right]=a_{\left(v_{2}\right)_{i-1, j}^{k-1}}^{2}\left[c_{2}, c_{2}-e_{i}-e_{k}\right]=a_{x} a_{\zeta} g_{6}^{V_{x \zeta}} \\
& V_{i+1, j}^{k-1} a_{28}^{2}\left[c_{2}, c_{2}+r_{28}^{2}\right]=a_{\left(v_{2}\right)_{i+1, j}^{k-1}}^{2}\left[c_{2}, c_{2}+e_{i}-e_{k}\right]=a_{\zeta} c_{x} g_{6}^{V_{x \zeta}} \\
& V_{i-1, j}^{k+1} a_{29}^{2}\left[c_{2}, c_{2}+r_{29}^{2}\right]=a_{\left(v_{2}\right)_{j-1, j}^{k+1, j}}^{2}\left[c_{2}, c_{2}-e_{i}+e_{k}=a_{x} c_{\zeta} g_{6}^{V_{x \zeta}}\right. \\
& V_{i+1, j}^{k+1} a_{30}^{2}\left[c_{2}, c_{2}+r_{30}^{2}\right]=a_{\left(v_{2}\right)_{i+1, j}^{k+1}}^{2}\left[c_{2}, c_{2}+e_{i}+e_{k}=c_{x} c_{\zeta} g_{6}^{V_{x \zeta}}\right. \\
& V_{\mathrm{i}, \mathrm{j}-1}^{\mathrm{k}-1} a_{31}^{2}\left[c_{2}, c_{2}+r_{31}^{2}\right]=a_{\left(v_{2}\right)_{i, j-1}^{k-1}}^{2}\left[c_{2}, c_{2}-e_{j}-e_{k}\right]=a_{y} a_{\zeta} g_{5}^{V_{y \zeta}} \\
& V_{\mathrm{i}, j+1}^{\mathrm{k}-1} a_{32}^{2}\left[c_{2}, c_{2}+r_{32}^{2}\right]=a_{\left(V_{2}\right)_{j,+1}^{k-1}}^{2}\left[c_{2}, c_{2}+e_{j}-e_{k}\right]=a_{\zeta} c_{y} g_{5}^{V_{y \zeta}} \\
& V_{\mathrm{i}, j-1}^{\mathrm{k}+1} \cdot a_{33}^{2}\left[c_{2}, c_{2}+r_{33}^{2}\right]=a_{\left(\mathrm{v}_{2}\right)_{i, j-1}^{k+1}}^{2}\left[c_{2}, c_{2}-e_{j}+e_{k}\right]=a_{y} c_{\zeta} g_{5}^{V_{y \zeta}} \\
& V_{i, j+1}^{k+1} \cdot a_{34}^{2}\left[c_{2}, c_{2}+r_{34}^{2}\right]=a_{\left(v_{2}\right)_{i, j+1}^{k+1}}^{2}\left[c_{2}, c_{2}+e_{j}+e_{k}=c_{y} c_{\zeta} g_{5}^{V_{y \zeta}}\right.
\end{aligned}
$$

A numerical ice sheet - ice shelf 3D

\section{velocity field solution}

T. J. Reerink et al.

\section{Title Page}

\section{Abstract}

Introduction

Conclusions

References

Tables

Figures

14

I

4

Back

Close

\section{Full Screen / Esc}

Printer-friendly Version 


\section{GMDD}

2, 81-158, 2009

\section{A numerical ice sheet}

- ice shelf 3D

Table 7. Continued.

$$
\begin{aligned}
& w_{\mathrm{i}, \mathrm{j}-1}^{\mathrm{k}}: a_{35}^{2}\left[c_{2}, c_{3}+r_{35}^{2}\right]=a_{\left(v_{3}\right)_{i, j-1}^{k}}^{2}\left[c_{2}, c_{3}-e_{j}\right]=a_{y} g_{04}^{w_{y}}+a_{y} b_{\zeta} g_{04}^{w_{y \zeta}} \\
& W_{\mathrm{i}, j+1}^{\mathrm{k}}: a_{36}^{2}\left[c_{2}, c_{3}+r_{36}^{2}\right]=a_{\left(v_{3}\right)_{i, j+1}^{k}}^{2}\left[c_{2}, c_{3}+e_{j}\right]=c_{y} g_{04}^{W_{y}}+b_{\zeta} c_{y} g_{04}^{W_{y \zeta}} \\
& W_{i, j}^{k-1}: a_{37}^{2}\left[c_{2}, c_{3}+r_{37}^{2}\right]=a_{\left(v_{3}\right)_{i, j}^{k-1}}^{2}\left[c_{2}, c_{3}-e_{k}\right]=a_{\zeta} g_{04}^{w_{\zeta}}+a_{\zeta \zeta} g_{04}^{w_{\zeta \zeta}} \\
& W_{\mathrm{i}, \mathrm{j}}^{\mathrm{k}}: a_{38}^{2}\left[c_{2}, c_{3}+r_{38}^{2}\right]=a_{\left(v_{3}\right)_{i, j}^{k}}^{2}\left[c_{2}, c_{3}\right] \\
& =b_{\zeta} g_{04}^{W_{\zeta}}+b_{\zeta \zeta} g_{04}^{W_{\zeta \zeta}} \\
& W_{\mathrm{i}, \mathrm{j}}^{\mathrm{k}+1}: a_{39}^{2}\left[c_{2}, c_{3}+r_{39}^{2}\right]=a_{\left(\mathrm{v}_{3}\right)_{i, j}^{k+1}}^{2}\left[c_{2}, c_{3}+e_{k}\right]=c_{\zeta} g_{04}^{W_{\zeta}}+c_{\zeta \zeta} g_{04}^{W_{\zeta \zeta}} \\
& W_{\mathrm{i}, \mathrm{j}-1}^{\mathrm{k}-1} a_{40}^{2}\left[c_{2}, c_{3}+r_{40}^{2}\right]=a_{\left(v_{3}\right)_{i, j-1}^{k-1}}^{2}\left[c_{2}, c_{3}-e_{j}-e_{k}=a_{y} a_{\zeta} g_{04}^{W_{y \zeta}}\right. \\
& W_{\mathrm{i}, j+1}^{k-1}: a_{41}^{2}\left[c_{2}, c_{3}+r_{41}^{2}\right]=a_{\left(v_{3}\right)_{i, j+1}^{k-1}}^{2}\left[c_{2}, c_{3}+e_{j}-e_{k}=a_{\zeta} c_{y} g_{04}^{W_{y \zeta}}\right. \\
& W_{\mathrm{i}, \mathrm{j}-1}^{\mathrm{k}+1}: a_{42}^{2}\left[c_{2}, c_{3}+r_{42}^{2}\right]=a_{\left(v_{3}\right)_{i, j-1}^{k+1}}^{2}\left[c_{2}, c_{3}-e_{j}+e_{k}\right\} a_{y} c_{\zeta} g_{04}^{W_{y \zeta}} \\
& W_{i, j+1}^{k+1}: a_{43}^{2}\left[c_{2}, c_{3}+r_{43}^{2}\right]=a_{\left(v_{3} i_{i, j+1}^{k+1}\right.}^{2}\left[c_{2}, c_{3}+e_{j}+e_{k}\right\} c_{y} c_{\zeta} g_{04}^{W_{y \zeta}}
\end{aligned}
$$

\section{velocity field solution}

T. J. Reerink et al.

Title Page

\section{Abstract}

Conclusions

Tables

14

4

Back
Introduction

References

Figures

I

$>$

Close

\section{Full Screen / Esc}

Printer-friendly Version

Interactive Discussion 
Table 8. The 13 matrix coefficients $a_{s}^{\prime}(i j k)\left[c_{,}, c_{m}+r_{s}^{\prime}\right]$ for the third equation: $I=3,1 \leq s \leq 13$, which are the coefficients in front of the discretized variables in Eq. (D32). See for more explanation the caption of Table 6 . The down part of the table shows the definitions and the ranges of various indices, and gives the format of the solution vector and the RHS vector
GMDD

2, 81-158, 2009

\section{A numerical ice sheet - ice shelf 3D velocity field solution}

T. J. Reerink et al.

\section{Title Page}

Abstract

Introduction

$W_{\mathrm{i}, j}^{\mathrm{k}-1}: a_{11}^{3}\left[c_{3}, c_{3}+r_{11}^{3}\right]=a_{\left(v_{3}\right)_{i, j}^{k-1}}^{3}\left[c_{3}, c_{3}-e_{k}\right]=a_{\zeta} \zeta_{z}$

$W_{\mathrm{i}, \mathrm{j}}^{\mathrm{k}}: a_{12}^{3}\left[c_{3}, c_{3}+r_{12}^{3}\right]=a_{\left(v_{3}\right)_{i, j}^{k}}^{3}\left[c_{3}, c_{3}\right] \quad=b_{\zeta} \zeta_{z}$

$W_{\mathrm{i}, j}^{\mathrm{k}+1}: a_{13}^{3}\left[c_{3}, c_{3}+r_{13}^{3}\right]=a_{\left(v_{3}\right)_{i, j}^{k+1}}^{3}\left[c_{3}, c_{3}+e_{k}\right]=c_{\zeta} \zeta_{z}$

The central indices are given by:

$c_{1}=c_{1}(i j k)=\operatorname{cindex}(i j k)+1$

$c_{2}=c_{2}(i j k)=\operatorname{cindex}(i j k)+2$

$c_{3}=c_{3}(i j k)=\operatorname{cindex}(i j k)+3$

with

cindex $(i j k)=3(i-1)+3(j-1) N_{\mathrm{x}}+3(k-1) N_{\mathrm{x}} N_{\mathrm{y}}$

The $i j k$ directed shifts in matrix $A$ for side by side neighbours:

$e_{i}=3$

$e_{j}=3 N_{x}$

$e_{k}=3 N_{\mathrm{x}} N_{\mathrm{y}}$

The solution vector $V$ is related to the $3 D$ velocity field by:

$u(i j k) \equiv v_{1}(i j k)=v_{1}\left(c_{1}\right)=V\left(c_{1}\right)$

$v(i j k) \equiv v_{2}(i j k)=v_{2}\left(c_{2}\right)=V\left(c_{2}\right)$

$w(i j k) \equiv v_{3}(i j k)=v_{3}\left(c_{3}\right)=V\left(c_{3}\right)$

The right hand side vector $R H S$ is given by:

$R H S\left(c_{1}(i j k)\right)=\frac{2 \rho_{\text {ice }} g}{C^{\mathrm{uvw}}} \partial_{x} H_{S}$

$R H S\left(c_{2}(i j k)\right)=\frac{2 \rho_{\text {ice }} g}{C^{\mathrm{uvw}}} \partial_{y} H_{s}$

$R H S\left(c_{3}(i j k)\right)=0$
Conclusions

References

Tables

Figures

14

$>$ I

4

Back

Close

Full Screen / Esc

Printer-friendly Version

Interactive Discussion 
Table 9. The coefficients of the boundary conditions at the outer planes in the sparse matrix which solves the set of three coupled equations for $u, v$, and $w$. See Table 8 for the index definitions. As in Eq. (3.32), $W\left(H_{b}\right)=\partial_{t} H_{b}+M_{b}+u\left(H_{b}\right) \partial_{x} H_{b}+v\left(H_{b}\right) \partial_{y} H_{b}$. The equations contained by the first three lines in this table are Dirichlet conditions, whereas the rest of the table represents Neumann conditions (see Sect. 3.6). The correction terms in the scaled boundary conditions in Eqs. (5.12-5.14) are neglected, because the domain is assumed to be such large that the scaling does not change in horizontal directions anymore at the domain boundaries. See Fig. 2 for the orientation of the vertical $\zeta(k)$ axis. Notice that the order of applying these boundary conditions matters, they have to be applied in this top-down order.

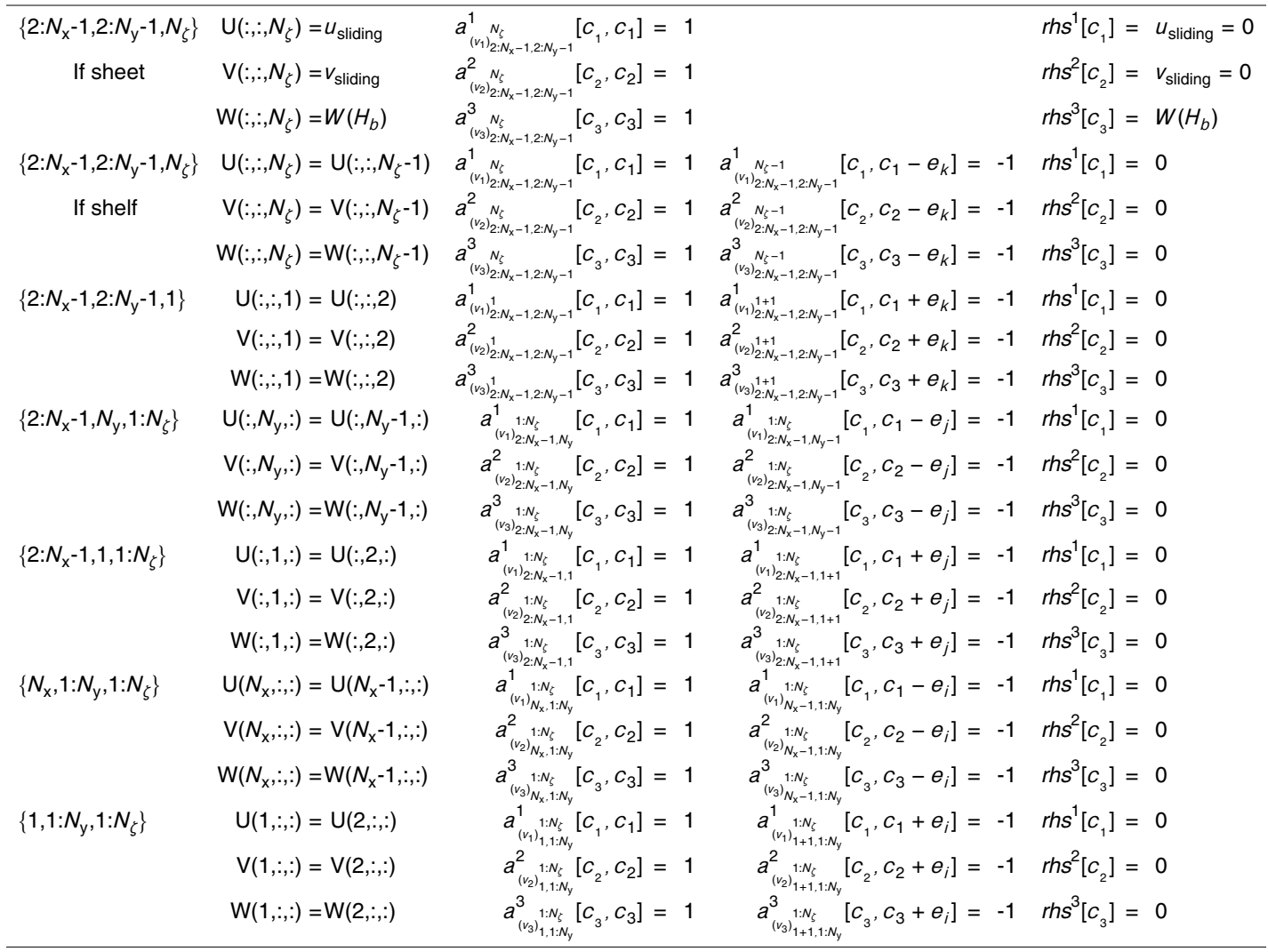

GMDD

2, 81-158, 2009

A numerical ice sheet - ice shelf 3D velocity field solution

T. J. Reerink et al.

Title Page

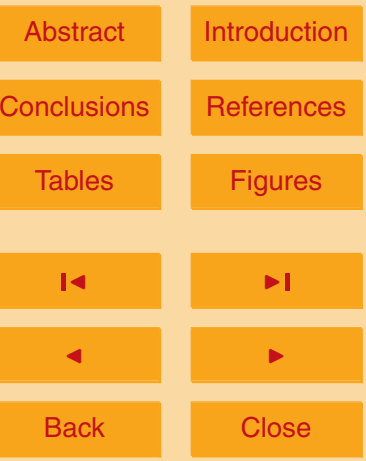

Full Screen / Esc

Printer-friendly Version

Interactive Discussion 

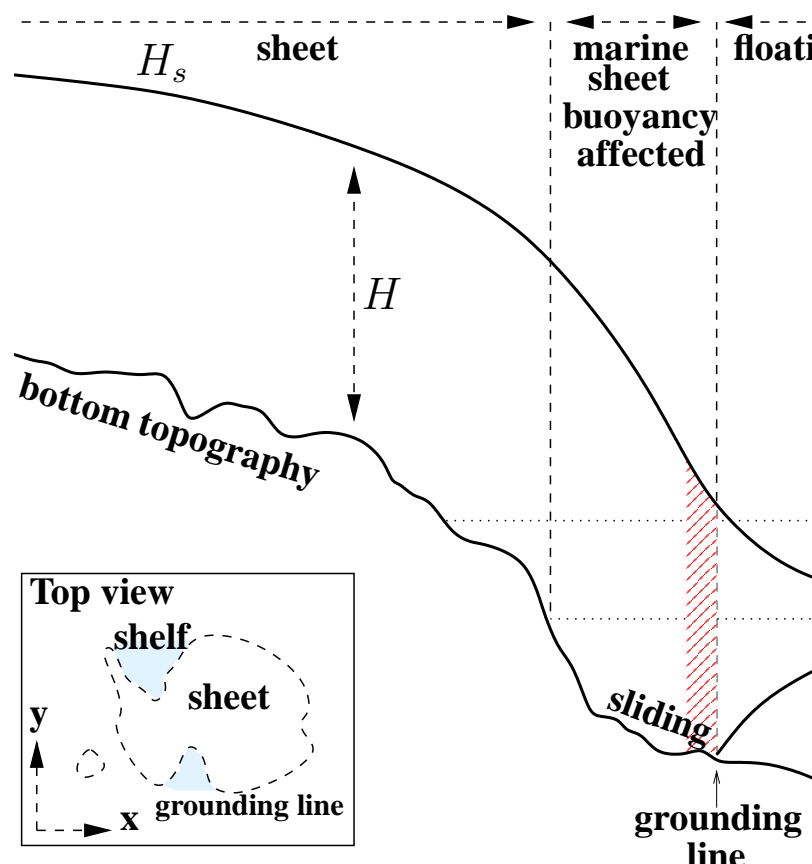

line

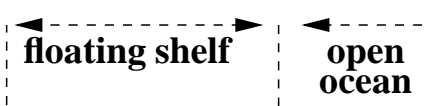

present day sea level sea level

Edge of shelf Calving front

bedrock

Cross section

Fig. 1. A sketch of a cross section of an ice sheet with an ice shelf. We call the part of the sheet near the grounding line where longitudinal stresses are important the transition zone. The transition zone is indicated by the red-hatched area. The ice thickness is indicated by $H$. The surface height $H_{s}$, the bedrock $H_{b}$ and the sea level $S$ are relative to a reference level: the present day sea level. The shelves are shaded blue in the inset.

A numerical ice sheet

- ice shelf 3D

velocity field solution

T. J. Reerink et al.

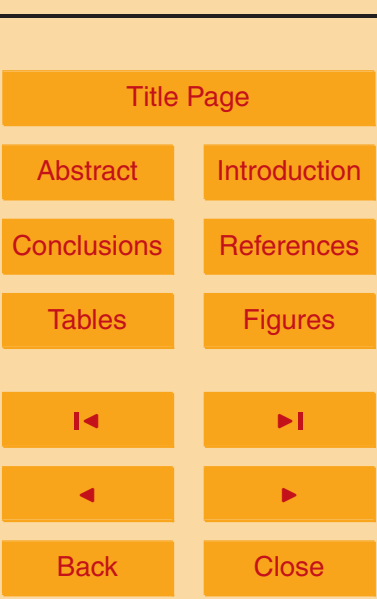

Full Screen / Esc

Printer-friendly Version

Interactive Discussion 


\section{GMDD}

2, 81-158, 2009

\section{typical situation}

atmosphere-ice interface

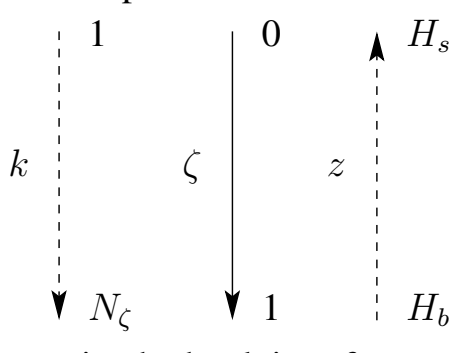

ice-bedrock interface

$$
\partial_{z} T<0
$$

$250 \mathrm{~K}$

$270 \mathrm{~K}$

$272 \mathrm{~K}$

Fig. 2. The non-equidistant vertically scaled axis $\zeta$ is directed oposite to the $z$ axis, and $\zeta$ is defined in Eq. (B8). The grid index $k$ and $\zeta$ are orientated equally. At the right, a typical Antarctic ice-cap temperature situation is indicated.
A numerical ice sheet

- ice shelf 3D

velocity field solution

T. J. Reerink et al.

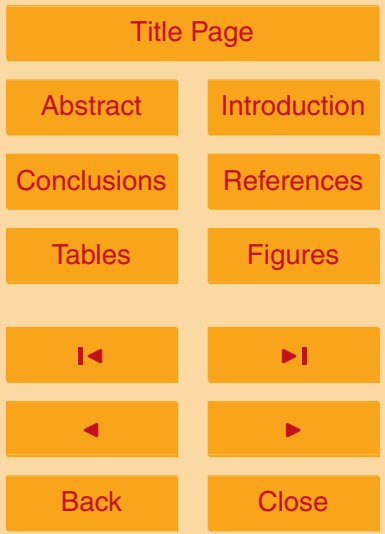

Full Screen / Esc

Printer-friendly Version

Interactive Discussion 